University of Louisville

ThinkIR: The University of Louisville's Institutional Repository

$5-2017$

\title{
Psychophysical analysis of three separate anisotropic patterns in vision : putting visual reference frames in conflict to study the class 1 and 2 oblique effects and the horizontal effect.
}

Eleanor O'Keefe

University of Louisville

Follow this and additional works at: https://ir.library.louisville.edu/etd

Part of the Psychology Commons

\section{Recommended Citation}

O'Keefe, Eleanor, "Psychophysical analysis of three separate anisotropic patterns in vision : putting visual reference frames in conflict to study the class 1 and 2 oblique effects and the horizontal effect." (2017). Electronic Theses and Dissertations. Paper 2631.

https://doi.org/10.18297/etd/2631

This Doctoral Dissertation is brought to you for free and open access by ThinkIR: The University of Louisville's Institutional Repository. It has been accepted for inclusion in Electronic Theses and Dissertations by an authorized administrator of ThinkIR: The University of Louisville's Institutional Repository. This title appears here courtesy of the author, who has retained all other copyrights. For more information, please contact thinkir@louisville.edu. 
PSYCHOPHYSICAL ANALYSIS OF THREE SEPARATE ANISOTROPIC PATTERNS

IN VISION: PUTTING VISUAL REFERENCE FRAMES IN CONFLICT TO STUDY

THE CLASS 1 AND 2 OBLIQUE EFFECTS AND THE HORIZONTAL EFFECT

\author{
By \\ Eleanor O'Keefe \\ B.S., UC Santa Barbara, 2010 \\ M.S., University of Louisville, 2013 \\ A Dissertation \\ Submitted to the Faculty of the \\ College of Arts and Sciences of the University of Louisville \\ In Partial Fulfillment of the Requirements \\ For the Degree of \\ Doctor of Philosophy \\ in Experimental Psychology
}

Department of Psychological and Brain Sciences

University of Louisville

Louisville, Kentucky

May 2017 

PSYCHOPHYSICAL ANALYSIS OF THREE SEPARATE ANISOTROPIC PATTERNS IN VISION: PUTTING VISUAL REFERENCE FRAMES IN CONFLICT TO STUDY THE CLASS 1 AND 2 OBLIQUE EFFECTS AND THE HORIZONTAL EFFECT

\author{
By \\ Eleanor O'Keefe \\ B.S., UC Santa Barbara, 2010 \\ M.S., University of Louisville, 2013
}

A Dissertation Approved on

April 14, 2017

by the following Dissertation Committee:

Dissertation Director

Dr. Paul DeMarco

Dr. Pavel Zahorik

Dr. Heywood Petry

Dr. Christian Stilp

Dr. Martha Bickford 


\section{ACKNOWLEDGEMENTS}

I would first like to acknowledge Dr. Paul DeMarco for his amazing support in enabling me to finish this project and receive my degree. I am especially thankful to Paul allowing me to continue the same dissertation project as well as helping me design the set-ups needed and make up the difference between project cost and my awarded grant funding. Many, many thanks to the other members of my dissertation committee: Dr. Pavel Zahorik, Dr. Heywood Petry, Dr. Christian Stilp, and Dr. Martha Bickford for taking their time to rigorously evaluate this project as well as answer my questions along the way. I would like to thank Dr. Edward Essock for inspiring the ideas behind this project. Thank you to all of the participants without whom I could not have gathered data, especially those who were available to participate in several conditions. Thanks to Dr. Kate Godwin, my previous lab mate who provided academic help and is a great friend. To Dr. Marci DeCaro who was an amazing TA advisor and helped me discover my love of teaching. Thank you to Dr. Cara Cashon who was always available to talk and for quick statistics questions. Thanks also to Dr. Edna Ross, Dr. Melinda Leonard, and Dr. John Pani who helped me with participant recruitment. Thank you to the Psychology department staff, especially Carla Gates, Diane Penrod and Kim Hosburgh. Thanks to the University itself and the Graduate school for making travel and project grants available and for graduate student appreciation week. To my parents who came out to visit at least once a year and gave me emotional support throughout the whole process. And lastly to my dog Samson, whose happy face greeted me at the door every day. 


\begin{abstract}
PSYCHOPHYSICAL ANALYSIS OF THREE SEPARATE ANISOTROPIC PATTERNS IN VISION: PUTTING VISUAL REFERENCE FRAMES IN CONFLICT TO STUDY THE CLASS 1 AND 2 OBLIQUE EFFECTS AND THE HORIZONTAL EFFECT

\section{Eleanor M. O'Keefe}

April 14, 2017
\end{abstract}

This dissertation describes and investigates a debate about differences in the visual system's anisotropic sensitivity to orientation as well as where and how the brain encodes visual orientation information. Three different visual perceptual patterns are discussed: the Class 1 and 2 oblique effects and the horizontal effect. The oblique effect is the ability to perceive cardinal orientations more easily than oblique orientations, and is found using narrowband stimuli. In the horizontal effect, oblique orientations are perceived most easily, horizontal orientations are perceived worst, and vertical orientations fall in-between, and is found using broadband stimuli.

While the majority of authors refer to the oblique effect as a whole, there is a case that it can be divided into Class 1 and Class 2 depending on what the task is measuring: low-level properties of the visual system or higher-level categorization and memory tasks. Moreover, when determining which reference frame the visual system uses to define the oblique effect, there is a disagreement as to whether it is the retinal or gravitational reference frame. To add evidence that there are two classes of the oblique effect and that they are defined by different reference frames (hence the disagreement), retinal, gravitational, and patterncentric coordinates were put in conflict with one another. The effects of phenomenological coordinates were also tested to determine whether top- 
down cognition played a role in either the Class 1 or Class 2 effect.

That the Class 1 oblique effect remained defined by retinal coordinates no matter the test. The Class 2 oblique effect was defined by gravitational coordinates during head tilt with no available patterncentric information. However, when patterncentric coordinates were available the Class 2 effect pattern was defined by these coordinates. Phenomenological coordinates seemed to make no difference to either effect. These results add to current evidence that the oblique effect should be separated into two classes: Class 1, which shows the low-level orientation characteristics of the visual system and is defined by retinal coordinates, and Class 2, which is defined by patterncentric coordinates, and gravitational coordinates when there is no patterncentric input.

There have been no studies to date to address the coordinates used by the visual system to define the horizontal effect. The horizontal effect is thought to be important for scene information, so the hypothesis was that it would follow patterncentric coordinates. Again, I tested retinal, gravitational, patterncentric, and phenomenological reference frames. During head tilt (no patterncentric information available), the standard horizontal effect did not remain tied to gravitational coordinates, nor did it transfer completely to retinal coordinates. The pattern did change completely with patterncentric coordinates during scene tilt, indicating that the horizontal effect is important for scene information. However, during scene tilt alone, retinal and patterncentric coordinates are aligned. During the head and scene tilt condition, when patterncentric and retinal coordinates are in competition, there was also incomplete transfer. Phenomenological changes seemed to have no effect. This suggests that the horizontal effect likely receives input from cortical 
areas where some cells update their receptive fields when vestibular information changes, but some do not, such as V2 or V3. 


\section{TABLE OF CONTENTS}

PAGE

ACKNOWLEDGEMENTS......................................................

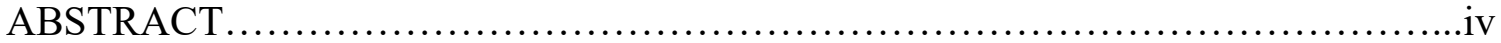

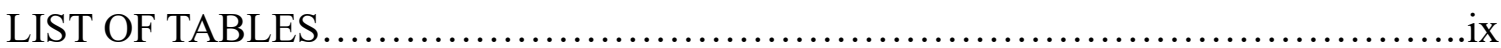

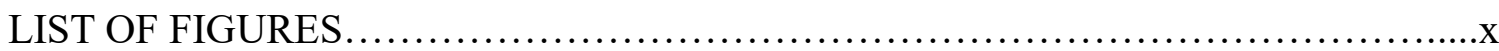

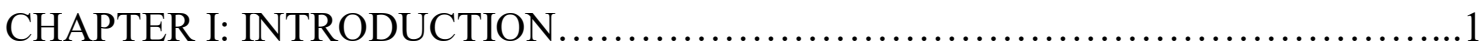

General Background on Vision............................................ 1

The Oblique Effect.................................................... 10

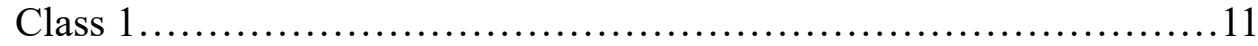

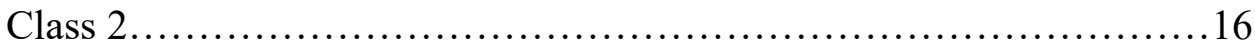

The Horizontal Effect................................................... 18

Visual Reference Frames...............................................23

Cortical implications............................................ 33

CHAPTER II: GENERAL METHODS AND ANALYSES $\ldots \ldots \ldots \ldots \ldots \ldots \ldots \ldots \ldots \ldots \ldots$

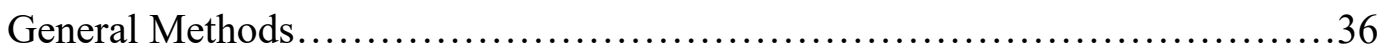

General Analyses........................................................... 37

CHAPTER III: EXPERIMENT 1: CLASS 1 OBLIQUE EFFECT $\ldots \ldots \ldots \ldots \ldots \ldots \ldots \ldots . \ldots . \ldots . \ldots$

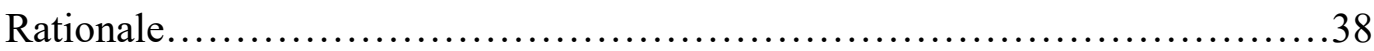

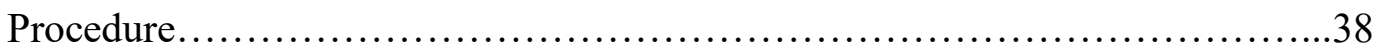

Analyses................................................................. 41

Results............................................................. 41

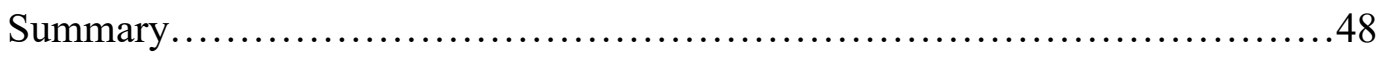

CHAPTER IV: EXPERIMENT 2: CLASS 2 OBLIQUE EFFECT ....................51

Rationale............................................................. 51

Procedure............................................................51

Analyses...........................................................53

Results............................................................ 53

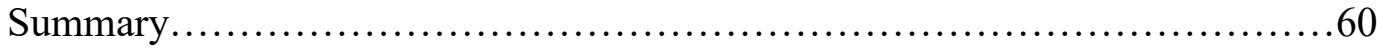

CHAPTER V: EXPERIMENT 3: THE HORIZONTAL EFFECT .....................63 
Rationale..........................................................63

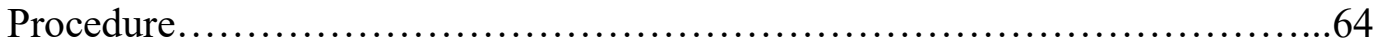

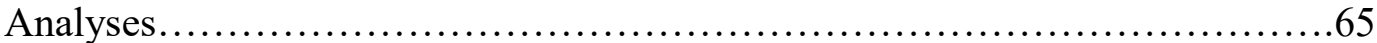

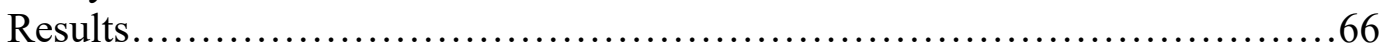

Summary ...................................................... 75

CHAPTER VI: GENERAL DISCUSSION ....................................79

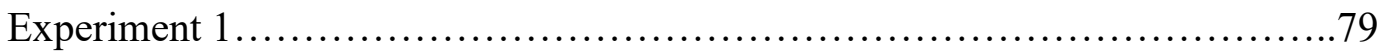

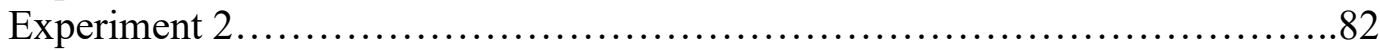

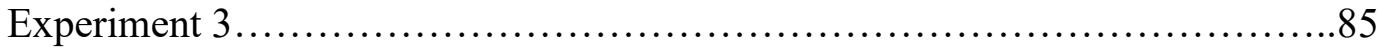

General Conclusions.................................................... 88

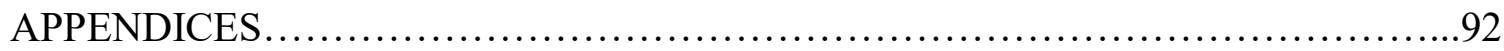

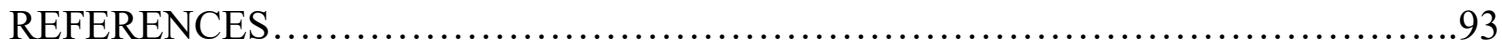

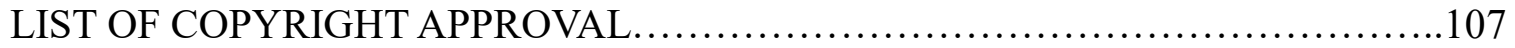

CURRICULUM VITA...................................................... 109 


\section{LIST OF TABLES}

TABLE PAGE

1. Class 1 oblique effect, head upright vs. head tilt means and standard deviations......42

2. Class 1 oblique effect, scene upright vs. scene tilt means and standard deviations....44

3. Class 1 oblique effect, head tilt: scene upright vs. scene tilt means and standard

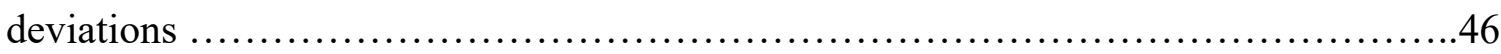

4. Class 1 oblique effect, top-down cognition means and standard deviations ..........47

5. Class 2 oblique effect, head upright vs. head tilt means and standard deviations......54

6. Class 2 oblique effect, scene upright vs. scene tilt means and standard deviations ....56

7. Class 2 oblique effect, head tilt: scene upright vs. scene tilt means and standard

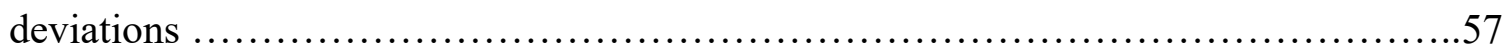

8. Class 2 oblique effect, top-down cognition means and standard deviations ..........59

9. Horizontal effect, head upright vs. head tilt means and standard deviations ..........67

10. Horizontal effect, scene upright vs. scene tilt means and standard deviations ........69

11. Horizontal effect, head tilt: scene upright vs. scene tilt means and standard deviations

12. Horizontal effect, top-down cognition means and standard deviations ..............73

13. Pairwise comparisons for main effect of orientation during no cognitive component

vs. top-down cognition component comparison. 


\section{LIST OF FIGURES}

FIGURE $\quad$ PAGE

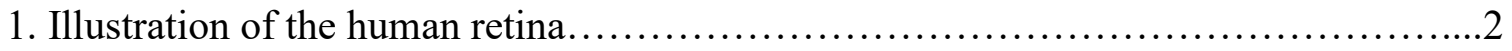

2. Standard gain control model..............................................6

3. Simple cell formation.....................................................

4. Examples of simple and complex orientation stimuli.......................... 19

5. Theoretical gain control model for early visual system......................... 21

6. Orientation content present in the natural environment...........................22

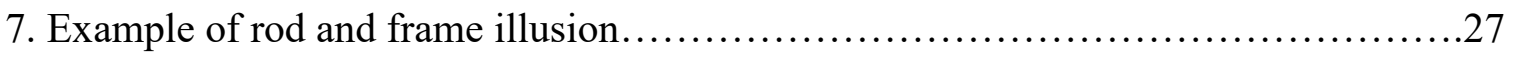

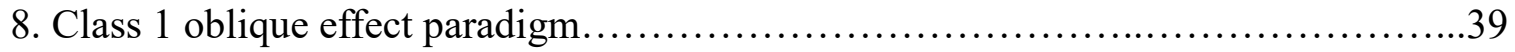

9. Head tilt apparatus ................................................... 40

10. Class 1 oblique effect, head upright vs. head tilt results........................43

11. Class 1 oblique effect, scene upright vs. scene tilt results.....................43

12. Class 1 oblique effect, head tilt: scene upright vs. scene tilt results...............46

13. Class 1 oblique effect, top-down cognition results........................... 48

14. Class 2 oblique effect paradigm .........................................52

15. Class 2 oblique effect, head upright vs. head tilt results......................55

16. Class 2 oblique effect, scene upright vs. scene tilt results.....................56

17. Class 2 oblique effect, head tilt: scene upright vs. scene tilt results...............58

18. Class 2 oblique effect, top-down cognition results.........................59 
19. Creation of stimuli used in the horizontal effect paradigm.

20. Horizontal effect, head upright vs. head tilt results............................68

21. Horizontal effect, scene upright vs. scene tilt results.........................70

22. Horizontal effect, head tilt: scene upright vs. scene tilt results....................71

23. Horizontal effect, top-down cognition results............................... 74 


\section{CHAPTER I}

\section{INTRODUCTION}

\section{General Background on Vision}

The visual process begins with light entering the eye through the lens. The lens bends this light in order to focus it on the tissue at the back of the eye called the retina. The retina itself is made up of layers of cells that process light signals, which are transmitted to the brain via the optic nerve. First, individual photons of light are detected by the photoreceptors, in the outermost layer of the retina farthest from the lens. The photons enter a photoreceptor through its outer segment where they pass through to the inner segment of the photoreceptor and are absorbed by photopigment. If the photon is absorbed, the photoreceptor is hyperpolarized, which signals cells in the next layer and so on, through the different layers of the retina (see Figure 1). This means that photoreceptors are the site of transduction in the visual pathway, as they convert the sensory signals of light photons into electrical impulses. To allow for perception over the large range of light intensities in the environment, these cells make use of divisive normalization (Carandini \& Heeger, 2012). Normalization allows the photoreceptors to shift their sensitivity relative to the average light intensity of the environment so that they can detect and respond to small changes in that environment.

There exist two major types of photoreceptors: rods and cones, which are responsible for scotopic (nighttime) and photopic (daytime) vision, respectively (Wandell, 1995). Cones are the only photoreceptors present in the fovea, a small area at 
the very back of the eye that provides the highest visual acuity. As one moves toward the periphery of the retina, the density of cones quickly decreases. While rods are not present in the fovea, their density steeply increases toward the periphery, peaks at around 15-20 degrees, and then slowly decreases. There are no photoreceptors present at the location where visual information leaves the eye (referred to as the blind spot). The majority of humans have three different cone-types, and each type contains a different photopigment

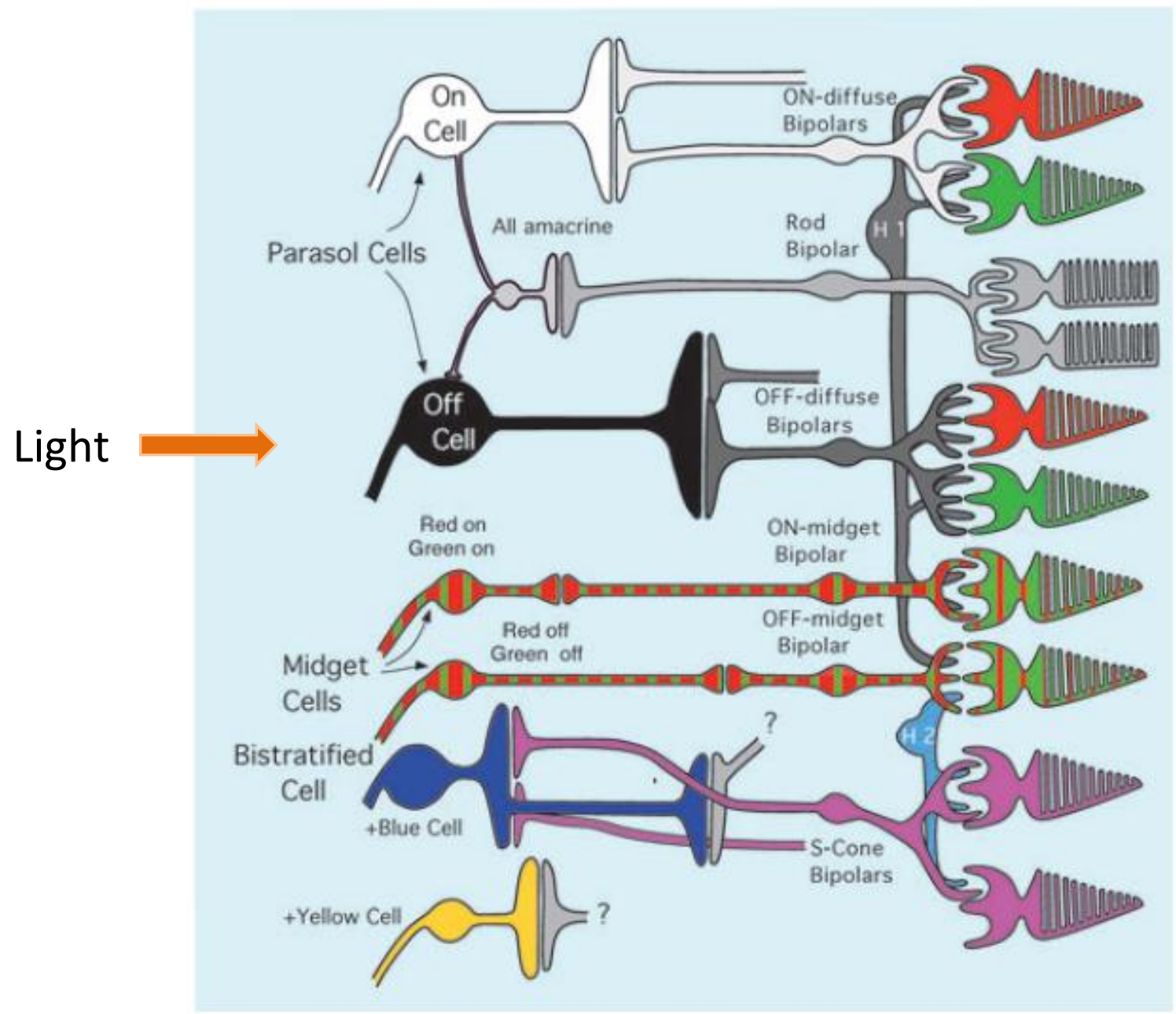

Figure 1. The human retina with its many cellular layers. Light enters from the left where it is first detected by the photoreceptor layer (adapted from Lee, 2011).

responsible for light absorption. These three types are referred to as $\mathrm{S}, \mathrm{M}$ and $\mathrm{L}$ cones as they best absorb light coming from short, medium, and long wavelengths of the visual 
spectrum. S cones only make up about 5 to $15 \%$ of the total number of cones and are not present in the fovea. $\mathrm{M}$ and $\mathrm{L}$ are randomly distributed within the confines of overall cone distribution, while the number of each varies widely across individuals. Cones synapse onto OFF and ON type bipolar cells in the next retinal layer, while rods synapse onto rod ON bipolar cells. In the fovea, a single M or L cone will synapse onto one ON and one OFF midget bipolar cell (Lee, Martin \& Grünert, 2010). This ratio changes to a few cones-to-one midget bipolar toward the periphery (Lee, 2011). The M and L cones that synapse onto diffuse bipolar cells and the S-cones that synapse on to blue cone ON bipolar cells, do so at a few-to-one ratio. Many rods synapse onto one bipolar cell, meaning that rod bipolar cells are more sensitive to light but transmit poorer spatial detail (Wandell, 1995). Horizontal and amacrine cells in the retina allow crosstalk between these different pathways.

While photoreceptor cells generally respond to points of light across the visual scene, most bipolar cells respond to larger spatial areas because of photoreceptor convergence. The receptive field of the bipolar cell, or the area of visual scene that will trigger a response from the cell, is usually characterized as a small circular field (the center) and a slightly larger circular field around the center (the surround). Both light in the center and darkness in the surround will excite the cell. Therefore, the stimulus that will cause the largest response in an ON-center cell is a small spot of light surrounded by darkness, which is why bipolar cells are referred to as having center-surround organization (Wandell, 1995). Note that the opposite organization also exists, wherein a cell's ideal stimulus is a dark spot surrounded by light (OFF-center cell). This means that even though this is early stage in the visual pathway, there are cells that are able to detect 
contrast in visual scenes. Some of these bipolar cells are linear processors, while others are spatially invariant at high spatial frequencies, that is, they respond to both stimulus onset and offset (Carandini, 2006; Shapley, 2009). These non-linear cells make use of contrast normalization in order to detect small changes in contrast as well as discount average background contrast (Carandini \& Heeger, 2012). Contrast normalization itself occurs through lateral neural communication, which results in the suppression similar neural information to help avoid redundancy (Schwartz \& Simoncelli, 2001).

The ON and OFF bipolar cells synapse onto ON and OFF ganglion cells, respectively. Diffuse bipolar cells synapse on to parasol ganglion cells (though some synapse on to small bistratified ganglion cells), midget bipolar cells synapse on to midget ganglion cells (at a 1:1 ratio in the fovea), and blue ON bipolar cells synapse on to small bistratified ganglion cells (referred to as K cells) (Lee et al., 2010). Ganglion cells also have center-surround organization and perform contrast normalization (Wandell, 1995). Their axons make up the optic nerve, which transmits visual information to the lateral geniculate nucleus (LGN). These axons are separated into two groups by the optic chiasm so that those cells that respond to the right visual field send information to the left LGN, and left visual field information is transmitted to the right LGN. Note that even though this separation occurs, retinotopic organization, or visual field mapping, is maintained in the LGN. The LGN itself is a layered structure and ganglion cell axons synapse in different layers depending on which eye they come from as well as their cellular morphology and electrophysiology. The midget and parasol ganglion cells synapse in separate layers: parvocellular, and magnocellular, respectively. The small bistratified ganglion cells synapse in layers referred to as koniocellular (Lee et al., 2010). The 
magnocellular pathway is thought to be responsible for red-green color vision and motion vision, while the parvocellular pathway seems to mainly transmit detailed spatial vision.

Neurons in the LGN have the same center-surround receptive field organization as ganglion cells, known as the classical receptive field (cRF). The center-surround model postulates that any input to the center is positive (can lead to excitation of the cell) and any input to the surround is negative (Carandini, 2004). Total input from the center and surround is then added and rectified such that any input below a threshold is considered zero input, while above threshold responses are linear. This is necessary because intracellular signals can be negative, so while input to a neuron can be below zero, a neuron itself sends an all-or-none response. However, this model cannot account for all behaviors of LGN cells, such as contrast saturation and masking, both of which involve suppression of the expected cellular response. Therefore, it has been suggested that in addition to the cRF, there must be a suppressive field in LGN cells (Carandini, 2004). The suppressive field is thought to occupy the same spatial location as the cRF and respond to the overall contrast of a stimulus. This is unlike the cRF itself, which has a different response if a stimulus switches from light to dark versus from dark to light. Input to the suppressive field never excites the cell, but serves only to suppress output. Gain control is a specific normalization model used to describe these cellular mechanisms. It can be thought of as an equation where the neuron's overall response is given by the output of the cRF divided by the output of the suppressive field (which is processing information in parallel) (see Figure 2; Carandini, 2004). 


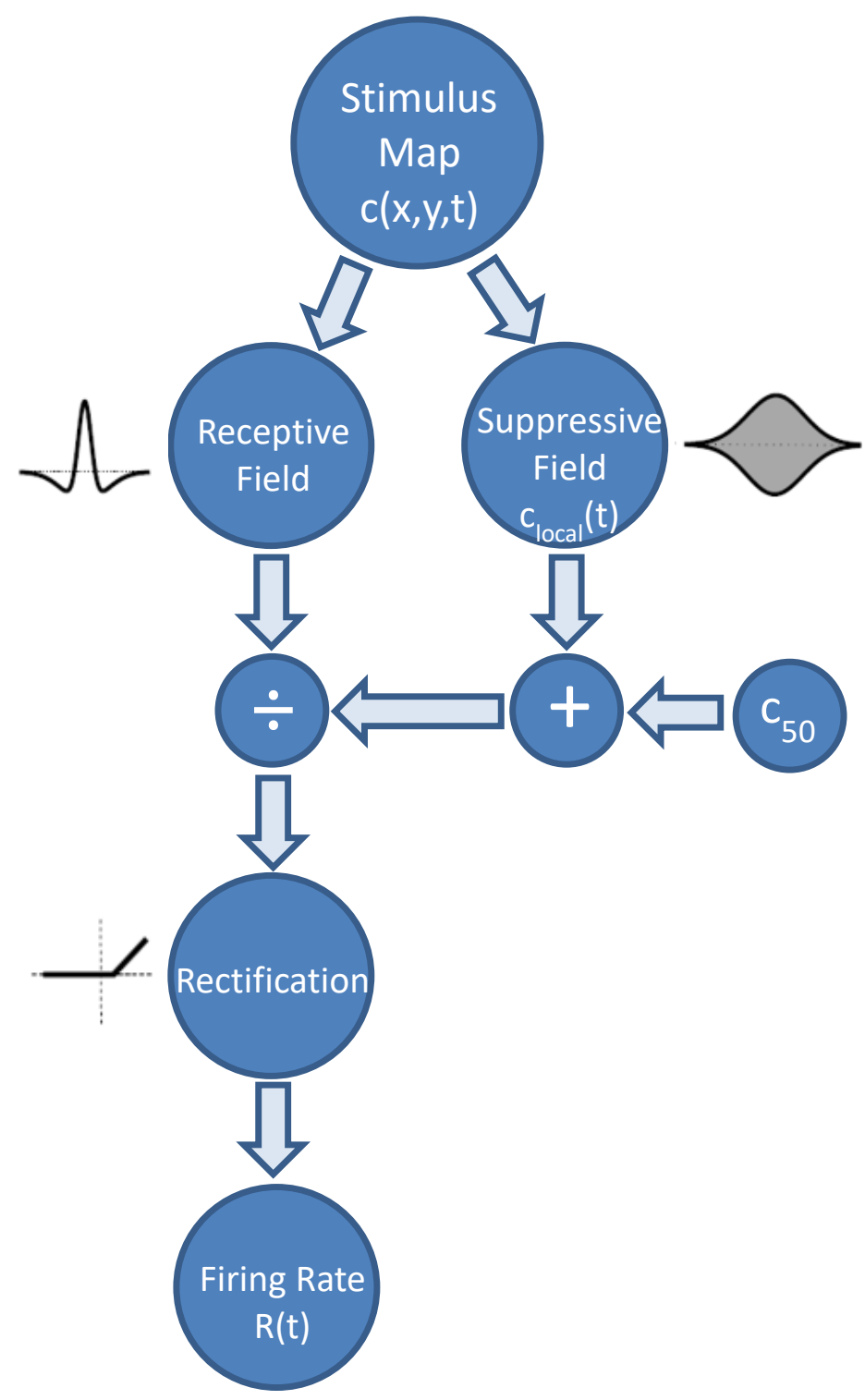

Figure 2. Model of gain control as computed by an LGN cell. The receptive field of the cell is excited by a stimulus. This stimulus is also detected by the cell's suppressive field (the output of the suppressive field is considered to be a measure of local contrast, $\mathrm{c}_{\text {local }}$. . The suppressive field is added to a constant, $\mathrm{c}_{50}$, to avoid dividing by zero. The output of the receptive field is then divided by the output of the suppressive field. This result then goes through half-wave rectification so that responses below zero are null, and responses above zero are linear (adapted from Carandini, 2004). 


\section{LGN}
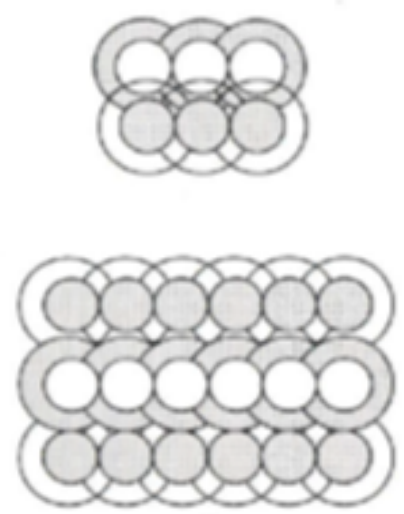

V1

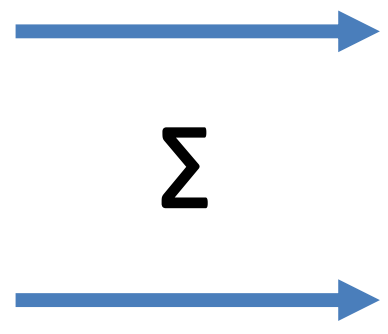

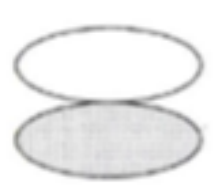

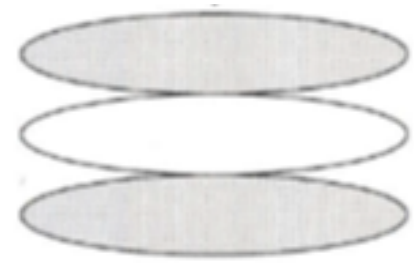

Figure 3. How outputs from many LGN neurons (left) can be combined to form a simple cell (right) (adapted from Wandell, 1995, p.167).

The next structure along this main visual pathway is the primary visual cortex (V1). Like the LGN, V1 is composed of layers of cells. Layers 2, 3 and 4 receive input from LGN neurons (Wandell, 1995). In V1, most neurons can be categorized as simple, complex, and hypercomplex, and these cells are arranged into columns as defined by their orientation preference (Hubel \& Wiesel, 1968). Simple cells have much more ovular shaped receptive fields so that, unlike the circular cells in the LGN, one side of the receptive field is longer than the other. These cells are thought to be made up of the output of many slightly overlapping LGN cells joined together (see Figure 3). In fact, recent work has shown that the orientation preference of an orientation column can be predicted by assessing the orientation preference of the corresponding population of afferent cells in the LGN (Jin, Wang, Swadlow \& Alonso, 2011). However, this is somewhat more complex than the simple diagram in Figure 3 in that the LGN cells sending feedforward information aren't arranged in a line, but in separate ON and OFF subregions. The optimal stimulus of a simple cell is a light bar at a specific orientation 
flanked by one or two dark bars (some cells respond best to a dark bar flanked by light bars). Like center-surround cells, simple cells have suppressive fields, but, unlike in the LGN, the suppressive fields in V1 have larger radii than their corresponding receptive fields. Also, it is important to note that in V1 different parts of the suppressive field process stimuli differently: the part of the suppressive field that overlaps the receptive field weighs all orientations equally, but the part of the suppressive field that is outside the receptive field weighs orientations similarly to the receptive field, (Carandini, 2004). And, while suppression in the LGN can be modeled well using a Gaussian envelope, simple cell suppression is largely asymmetric. Luckily, gain control is still a satisfactory method to model simple cells, but these differences from center-surround cells must be taken into account when modeling. Normalization models can also be used to describe populations of neurons, where the normalization factor is weighted depending upon the sensitivities of a group of neuron's receptive and suppressive fields (Carandini \& Heeger, 2012). It must be noted that the overall responses of V1 simple cells are not only due to feedforward connections from the LGN, but also to lateral and feedback connections (Priebe \& Ferster, 2012).

Complex cells have similar receptive fields to simple cells, but they respond to an optimally-oriented line anywhere in the receptive field, not in a specific ON region. That is, they are spatially insensitive and also size insensitive, (Carandini, 2004). About half of all complex cells have a directional preference for the way in which stimuli pass through their receptive fields. These direction-selective cells are found in layers 4 and 6 of V1. Hypercomplex cells are sometimes referred to as end-stopped cells, as they respond optimally to oriented lines of specific lengths. Simple, complex, and hypercomplex cells 
are all responsible for encoding low-level orientation contours and therefore indicate that V1 must play an important role in the visual effects the current work will be testing.

Cells in V1 make up multiple orientation "channels". An orientation "channel" is made up of all of the cells with receptive fields that respond maximally to one particular orientation, but respond to different spatial locations across the visual field. The channels are narrowly tuned, meaning that they are sensitive to only a limited range of orientations. That is, they respond maximally to a single orientation, somewhat less to neighboring orientations and not at all to orientations a given rotation away from their preferred orientation (dependent on their specific bandwidth). A channel responds passes on orientation information - if the stimulus contains orientation contours that fall within the channel's bandwidth (DeValois, 1988).

It is important to consider the types of stimuli that can be used to elicit responses of these types of cells in V1. More and more researchers are using visual stimuli that mimic the low-level statistics present in the natural world. Simple stimuli, such as oriented sinusoidal gratings and Gabor patches (gratings multiplied by a Gaussian envelope) are useful because they are easily quantifiable, and, therefore, the output (participant responses) are more straightforward. In this way, it is easier to test whether the input/output relationship is linear. However, more complex stimuli, such as natural scene images, are important to use as well to gain insight into how the visual system interprets the more complex stimuli that are encountered in the real world. Using this type of stimuli has shown that the assumption of linearity does not usually hold (Kayser, Kording \& Konig, 2004). That is, the way the visual system responds to a complex image cannot be predicted based on responses to simple stimuli. For example, about $60 \%$ of 
neurons show the suppressive effect with natural images, as their responses are more muted when a bar is flashed on a natural image background than when it is presented against a grey background, (MacEvoy, Hanks, \& Paradiso, 2008). Both simple and complex neurons in V1 respond differently to natural scenes and simple grating stimuli (David, Vinje \& Gallant, 2004). And while models formed from natural scene responses can accurately predict neuronal responses to natural movies, models generated from grating responses are significantly less accurate, (David et al, 2004). Natural stimuli were used to show that in addition to the $\mathrm{CRF}$ and suppressive surround, both spiking history and local field potential account for a neuron's response (Haslinger et al, 2012). A generalized linear model with the addition of these two components accounted for more variance when applied to neural responses to grating stimuli than responses to natural stimuli. This shows that models made using natural stimuli can apply to grating stimuli, and that neural responses to natural scenes are much less predictable than responses to gratings. These differing responses to natural scene stimuli are important considerations that will be addressed in the context of the horizontal effect later in this paper.

\section{The Oblique Effect}

The visual system functions in such a way that specific orientations are perceived better than others. For example, when presented with simple, oriented line stimuli, it is easier to perceive cardinally-oriented than obliquely-oriented stimuli. This finding is termed "the oblique effect" (Appelle, 1972). This effect has been found using many experimental tasks, such as detection, discrimination, orientation reproduction, and judgment of line orientation, and it has been seen across many species including 
octopuses, goldfish, rabbits, rats, squirrels, cats, chimpanzees, macaques, and of course, humans, including children (see Appelle, 1972 for summary; Berman, 1976; Williams, Boothe, Kiorpes \& Teller, 1981; Essock \& Siqueland, 1981, but see Teller, Morse, Borton $\&$ Regal, 1974). While not all authors agree, there is some evidence that the oblique effect can be split into two classes as it is believed to occur at different levels in the visual system depending on what the task itself is measuring, (Essock, 1980; Luyat \& Gentaz, 2002; Smyrnis, Mantas \& Evdokimidis, 2014).

\section{Class 1 Oblique Effects}

Class 1 oblique effects are found in tasks involving early visual processing specifically, such as contrast threshold and acuity (Essock, 1980; Luyat \& Gentaz, 2002). While the Class 1 oblique effect occurs early in the visual pathway, it is believed to be a post-retinal phenomenon (Campbell, Kulikowski \& Levinson, 1966; Frost \& Kaminar, 1975). Campbell et al (1966) bypassed the lens using interference fringes and found that the resolution and sensitivity for cardinal orientations were still better than for obliquelyoriented gratings. Tyler and Mitchell (1977) showed that the oblique Class 1 effect is not the same as optical blur using a Vernier acuity task. Compared to clearly visible vertical stimuli, there was a reduction in sensitivity at high spatial frequencies only with vertical blurred stimuli, while sensitivity was reduced across all spatial frequencies with clear oblique stimuli. Both Frost and Kaminar (1975) and Maffei and Campbell (1970) recorded human averaged evoked potentials (EVPs) from the occipital area of the scalp, and found a greater response to cardinally-oriented phase-reversed gratings than obliques; Maffei and Campbell report no difference between retinal EVP responses to cardinal and 
oblique gratings. It has even been suggested that the oblique effect is an unconscious bias, as the pattern still holds with low-visibility stimuli, (deGardelle, Koudier and Sackur, 2010). All of these results lead to the early cortical visual system being implicated as the site of the oblique Class 1 effect.

The cause of these anisotropies is thought to be due mainly to the difference in populations of neurons in the early visual system (primary visual cortex or areas nearby). It has been reported that more cells in these areas are tuned to cardinal orientations than oblique orientations, and that the cells tuned to cardinals have sharper tuning curves relative to those tuned to obliques (Rose \& Blakemore, 1974; Furmanski \& Engle, 2000; Li, Peterson \& Freeman, 2003; (foveally) Mansfield, 1974; Mansfield \& Foster, 1978). A higher number of cardinally-tuned neurons with narrower tuning curves leads to greater signal strength when stimuli are oriented horizontally or vertically. However, many studies still show discrepancies of the neuronal population response and specifically, where it occurs. Li et al (2003) note that studies have both found and not found differences in numbers of cells tuned to cardinal versus oblique orientations in single cell and VEP studies, and both found and not found differences in orientation tuning specificity for cells tuned to cardinal versus oblique orientations. These authors theorized that this could be due to anisotropies only being exhibited in a subpopulation of neurons, and therefore examined a few thousand cells in cat area 17 (thought to correspond to primate area V1). They found a greater anisotropy in simple cells (as opposed to complex and hypercomplex), both in regards to a greater number of cells tuned to cardinal orientations, as well as significantly narrower tuning curves at horizontal orientations (while cells tuned to vertical had similar tuning widths as those tuned to 
obliques). Cat area 18 (corresponds to V2) has also been studied: using optical imaging, a significantly larger region was found to respond to cardinally oriented gratings as compared to obliquely oriented gratings (Wang, Ding \& Yunokuchi, 2003). These authors also found that the areas responding to obliquely oriented gratings responded to a larger range of stimuli, implying larger bandwidths. When Chapman and Bonhoeffer (1998) used optical imaging to study the orientation tuning of cells in ferret area 17 and discovered a majority of cells were tuned to cardinal orientations, but that the overrepresentation varied significantly across individual ferrets.

Studies have also assessed changes in orientation tuning that may occur during development. Leventhal and Hirsch (1975) found that cats had to be reared in an environment that contained diagonal lines in order to develop visual cortical neurons that preferentially respond to diagonal contours, whereas the cats developed neurons with preferential responses to horizontal and vertical lines no matter the rearing environment. Both Blakemore and Cooper (1970) and Hirsch and Spinelli (1970) raised kittens in environments with only specific orientations present, and found that the majority of corresponding cortical cells responded best to those orientations. It logically follows that V1 cells somewhat mirror our natural environment, and the natural environment contains a greater amount of information at horizontal than any other orientation (see The Horizontal Effect below). Tanaka, Tani, Ribot, O’Hashi and Imamura (2009) also studied orientation plasticity during feline development. They found a prominent bias of neurons tuned to horizontal before postnatal day 35 that eventually became a weak vertical bias through the natural developmental process. Cats were not the only animals studied. Shen, Tao, Zhang, Smith and Chino (2014) measured preferred orientation of V1 and V2 
neurons in infant macaque monkeys to compare to their previous work in adult macaques. In the adult macaque, they found no orientation preferences across V1, while $56 \%$ of cells in V2 preferred cardinal orientations, and between the cardinals, preference for horizontal was the highest. Infant macaques showed the same trend, but the bias was weaker than in adults. These authors concluded that the orientation bias in V2 depends on prenatally-determined cortical circuits that are then strengthened by visual experience. Whether the difference in orientation preference was found in V1 or V2, it seems that rearing environment influences the final orientation tuning of cells in early visual cortex.

Of course, single-cell approaches represent only one of several kinds of studies that have been performed to determine orientation preference, and one must consider that varying methodologies may contribute to the varying conclusions regarding orientation population coding in the occipital cortex. Kamitani and Tong (2005) were able to use fMRI activity patterns from pooled voxels in V1 and V2 to correctly predict the orientation of the grating stimuli subjects had been viewing, but they found no orientation preference in these areas. Swisher et al (2010) used multivariate pattern analysis and high-resolution fMRI to show that the majority of orientation information coding can be found in V1 at spatial scales ranging from individual orientation columns (1mm) to about a centimeter. Unlike Kamitani and Tong (2005), their results show a greater response to oblique orientations than to cardinal orientations. M. Sun et al (2013) used functional near-infrared spectroscopy to measure the oxygenated hemoglobin response and also found the magnitude of response to be greatest in response to obliquely oriented gratings, though the differences were only significant in the left occipital lobe. This anisotropic response was also found in a greater blood-oxygen-level-dependent (BOLD) signal for 
obliques by Mannion, McDonald and Clifford (2010) in V1, V2, V3 and V3A/B using oriented sinusoidal gratings as stimuli. Nasr and Tootell (2012) tested the idea that the orientation bias arises at a higher visual area than V1/V2. They used indoor and outdoor scenes, overlapping squares, and arrays of simple line segments as stimuli and found that only the parahippocampal place area (PPA) showed a cardinal bias in response to any of these stimuli (simple and complex). Therefore, these authors argue that there is an analog to the perceptual oblique effect in the PPA for scene processing, though they do note that they were averaging activity in V1 across multiple orientation columns, which may not have been a sensitive enough technique to see any clear orientation bias.

Freeman, Brouwer, Heeger and Merriam (2011) argue that the results of conventional orientation decoding from fMRI scans don't actually reflect individual orientation columns, but are instead due to larger-scale orientation maps in V1. Their results showed a weak vertical bias, though the mapping was less clear near the fovea. Therefore, it is possible that orientation biases found in the above fMRI studies may be due to these coarse orientation maps and not individual orientation columns. However, it is hard to say whether or not the biases in the coarse maps are also present in the underlying columns, and whether columns or large scale maps are responsible for the oblique effect. Other work contends that fMRI can be used to look at orientation columns in humans when using an unconventional spin echo method, versus the usual gradient echo sampling (Yacoub, Harel \& Ugurbil, 2008). With this technique, these authors found that a significantly larger number of voxels showed a preference for vertical, though they found no difference in bandwidth size across preferred orientation. Additionally, singlevoxel orientation preference in $\mathrm{V} 1$ has been found to be predominantly cardinal, which is 
measured at a smaller size than the larger-scale orientation maps (P. Sun et al, 2013). So while those who measure coarse orientation biases seem to find oblique biases in early visual cortex, those using methods that measure smaller areas, and perhaps individual orientation columns, tend to find vertical and cardinal biases.

\section{Class 2 Oblique Effects}

The Class 2 oblique effect is present in higher level processing tasks such as orientation identification, reaction time, memory, discrimination, symmetry and illusions (Essock, 1980; Heeley \& Buchanan-Smith, 1992; Leone, Lipshits, Gurfinkel \& Berthoz, 1995; Westheimer, 2003; deGardelle et al, 2010; Corbett \& Carrasco, 2011). The Class 2 effect is thought to be due to trouble distinguishing between different oblique orientations psychophysically, while horizontal and vertical orientations remain easily discernible. This effect has been seen in humans as young as two months old, where they confuse mirror-image oblique orientations, but are able to discriminate between horizontal and vertical orientations (Essock \& Siqueland, 1981). In a seminal paper, Essock (1980) used three separate psychophysical tasks to show the difference between Class 1 and 2 oblique effects. Neither orientation categorization (cardinal versus oblique) nor orientation detection tasks' findings varied across orientation, but the orientation identification task $\left(0,45,90\right.$, or $\left.135^{\circ}\right)$ produced differences in reaction time across orientation. In the identification task, participants were having trouble discriminating between oblique images, while there was no such issue when they had to discriminate between horizontal and vertical. If the results were simply due to low-level visual processing, the oblique effect found in the identification task would have also been found in the categorization task. While other authors may refer to their findings as supporting the general oblique 
effect, it is easy to see that their tasks involve more top-down processing and can be categorized as Class 2. Heeley and Buchanan-Smith (1992) found just noticeable differences (JNDs) to be larger for obliquely-oriented gratings than for cardinal orientations in a clockwise discrimination task. In particular, JNDs were much larger for obliques in a two-interval forced-choice (2IFC) format than in the two-alternative forcedchoice format (2AFC). This implies that there is a cognitive component to the oblique effect, as short-term memory for oblique orientations is not as precise as that for cardinal orientations. Line segments spaced out to facilitate amodal completion show an oblique effect that is also stronger during 2IFC tasks than 2AFC tasks (Westheimer, 2003). It should be noted that the effect is present even though part of the stimulus would not be detected by low-level receptive fields. Virtual lines whose ends are marked by arrow heads and circles (with a dot in the middle) also elicited the oblique effect in the clockwise discrimination task. Strength of the effect increased as component separation increased, and was still present at a distance of $20^{\circ}$ apart, which is larger than the size of any RFs in V1, meaning that there must be top-down feedback. Reaction times are shorter for judgements of symmetry when objects are symmetrical about a cardinal axis instead of about an oblique axis, a task that also employs higher-level cognition (Leone et al, 1995). Smyrnis et al., (2014) found that when participants had to adjust an arrow to point toward an on-screen target, not only was there less variance when the target was on or near a cardinal axis (Class 1), but by measuring the gain (change in mean directional error with change in target direction) they also found a space expansion near cardinal orientations and retraction near oblique orientations, meaning that participants were much 
better at categorizing cardinals (Class 2). These results suggest that both classes of the oblique effect can play a role in one task, but they are not necessarily additive.

The origin of the Class 2 oblique effect is less clear than that of Class 1, though it definitely occurs higher in the visual processing stream as it is affected by scene processing. In an orientation categorization task, Mikhailova, Gerasimenko, Krylova, Izyurov and Slavutskaya (2015) found no orientation selectivity in early evoked potentials at $\mathrm{P} 1$ in the occipital cortex, but did in the parietal cortex, which is known to process spatial information. At N1, which occurs 150 to $200 \mathrm{~ms}$ post-stimulus there was a difference in the occipital cortex, though this most likely occurred due to feedback as there was no difference found in this lobe at the earlier (100ms post-stimulus), P1, component. Luyat and Gentaz (2002) note the Class 2 effect could be caused by the general definition of space in that cardinal orientations are typically used to form a reference frame and encode oblique orientations in relation to it. Others suggest something like this in terms of 2-D directional space where information is represented categorically based on a cardinal coordinate system, that is perhaps shared by modalities and not specific to vision, as an oblique effect also occurs in haptics (Baud-Bovy and Gentaz, 2012; Smyrnis et al, 2014).

\section{The Horizontal Effect}

As opposed to stimuli used in oblique effect tasks, stimuli that are broadband, both in terms of in spatial frequency and orientation content (see Figure 4), produce lowest thresholds for oblique and highest for horizontal orientations. Thresholds for vertical orientations fall intermediate between those for oblique and horizontal 
orientations. This is known as the horizontal effect. It has been found in contrast matching tasks, contrast threshold tasks, oriented content detection and contrast masking (Essock, DeFord, Hansen \& Sinai, 2003; Hansen \& Essock, 2004; (masking with small SOAs) Essock, Haun \& Kim 2009; (overlay and surround masking) Kim, Haun \& Essock, 2010). That is, with broadband stimuli, humans are best able to detect oblique orientations, then vertical, and lastly horizontal.
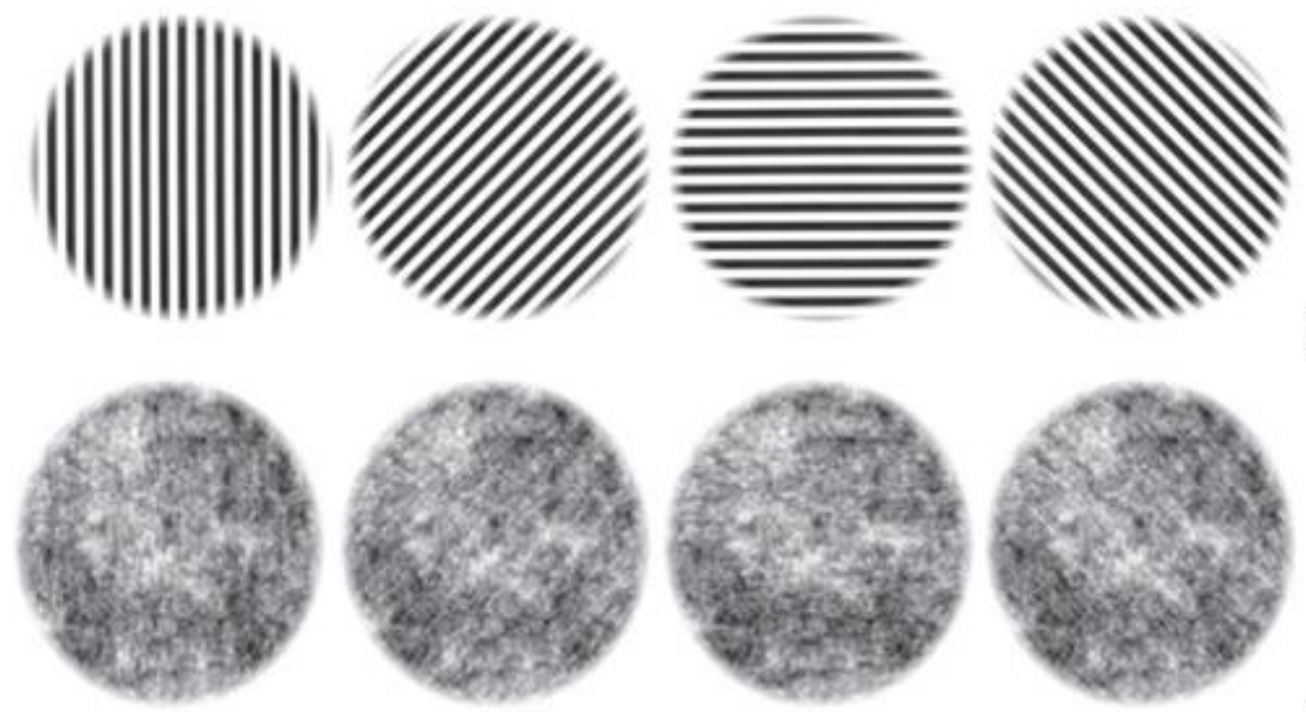

(a)

(b)

Figure 4. Example stimuli that will elicit (a) the Class 1 oblique effect at threshold: square wave gratings oriented at vertical, $45^{\circ}$, horizontal, and $135^{\circ}$. Example stimuli that will elicit (b) the horizontal effect at threshold: 1/f noise images with orientation increment. (Hansen \& Essock, 2004).

The cause of the horizontal effect is most likely due to contrast gain control, a type of contrast normalization. In this case, the result of gain control is to divide (normalize) a single neuron's response by the pooled response of neurons that are similarly tuned in orientation or spatial frequency (Graham \& Sutter, 2000). Therefore, when broadband stimuli are used, many more neuronal responses are present in the normalization pool. As applied to the horizontal effect, the gain control model shows that 
the divisive signal is greater for cardinal orientations (see Figure 5). This is because of the greater number of neurons tuned to cardinals as well as a Gaussian-weighted pool that itself is anisotropic with more weight given to horizontal contours and less to oblique contours. More specifically, there are two different weights in the proposed gain control model for this effect: one added to the semi-saturation constant that is greatest at horizontal and least at vertical, and one added to masking strength (when a mask is present) that is greatest for cardinals. Both of these lead to the most suppression occurring at horizontal orientations (Essock, Haun \& Kim, 2009). Willmore, Bulstrode and Tolhurst (2012) used the Bienenstock, Cooper, and Munro (BCM) rules to show that when using natural image stimuli, contrast normalization was needed in the model in order to generate parameters of real V1 simple cells. The BCM Learning Rule uses Hebbian rules applied at synapses, focusing on temporal competition, to generate simple cell RFs (Bienenstock, Cooper \& Munro, 1982). That is, the greater magnitude of response of a postsynaptic neuron due to the input of a presynaptic neuron, the more capable the presynaptic neuron becomes at exciting the postsynaptic neuron in the future. And along with this, the lesser magnitude of a post synaptic neuron's response to a presynaptic neuron's input, the less capable the presynaptic neuron becomes at exciting the postsynaptic neuron. This leads to spatial competition between presynaptic neurons. The natural image model also produced a population of neurons with a bias toward cardinal orientations, resulting from the model learning from natural images, which themselves have a cardinal orientation bias (Willmore et al, 2012). 


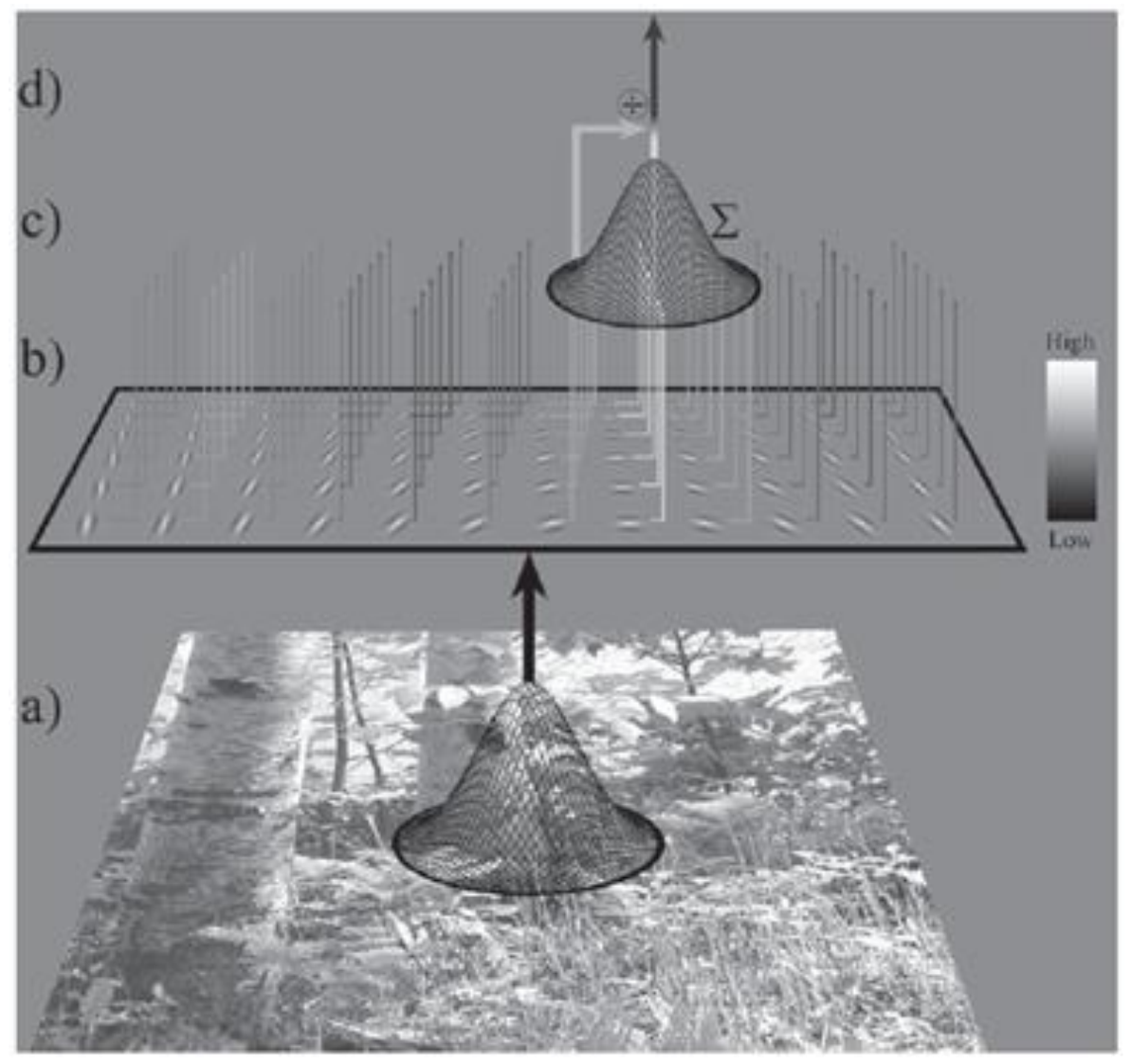

Figure 5. Gain control model (Hansen \& Essock, 2004). (a) Gaussian-weighted envelope of pooled neuronal activity when viewing a natural scene image. (b) Example neurons tuned in orientation and spatial frequency with most weight given to horizontal and least to oblique orientations. (c) Gaussian-weighted envelope of the summed neuronal response (numerator of gain control equation). (d) Final response of neuronal population after dividing by the overall activity.

The natural environment itself contains most orientation content (Fourier power) at horizontal, second most at vertical, and least at oblique orientations (see Figure 6) (Switkes, Mayer \& Sloan, 1978; Baddeley \& Hancock, 1991; Hancock, Baddeley \& Smith, 1992; Coppola, Purves, McCoy \& Purves, 1998; Keil \& Cristobal, 2000; Hansen \& Essock, 2004; Girschik, Landy \& Simoncelli, 2011). It is evident that a greater amount of content present at cardinal orientations would lead to a greater excitation of neurons tuned to cardinals. More suppression would also occur, leading to a comparatively 
weaker response, and therefore worse perception, at cardinal orientations. Gain control, operates here to suppress the most common information in the environment so that our perception is not overwhelmed by this redundant information and information content in the environment is perceived more equally, i.e. sometimes referred to as "whitening" the stimulus. The horizontal effect then, serves to whiten a natural scene by suppressing horizontal content the most and oblique input the least, which is a highly beneficial step in the coding process. This means that typical natural scene content is suppressed and less-prevalent content is made relatively more salient (Hansen \& Essock, 2004; Haun, Hansen, \& Essock, 2006; Essock et al, 2009; Haun \& Essock, 2010; Kim, Haun, \& Essock, 2010; see also Schwartz \& Simoncelli, 2001; Wainwright, 1999).

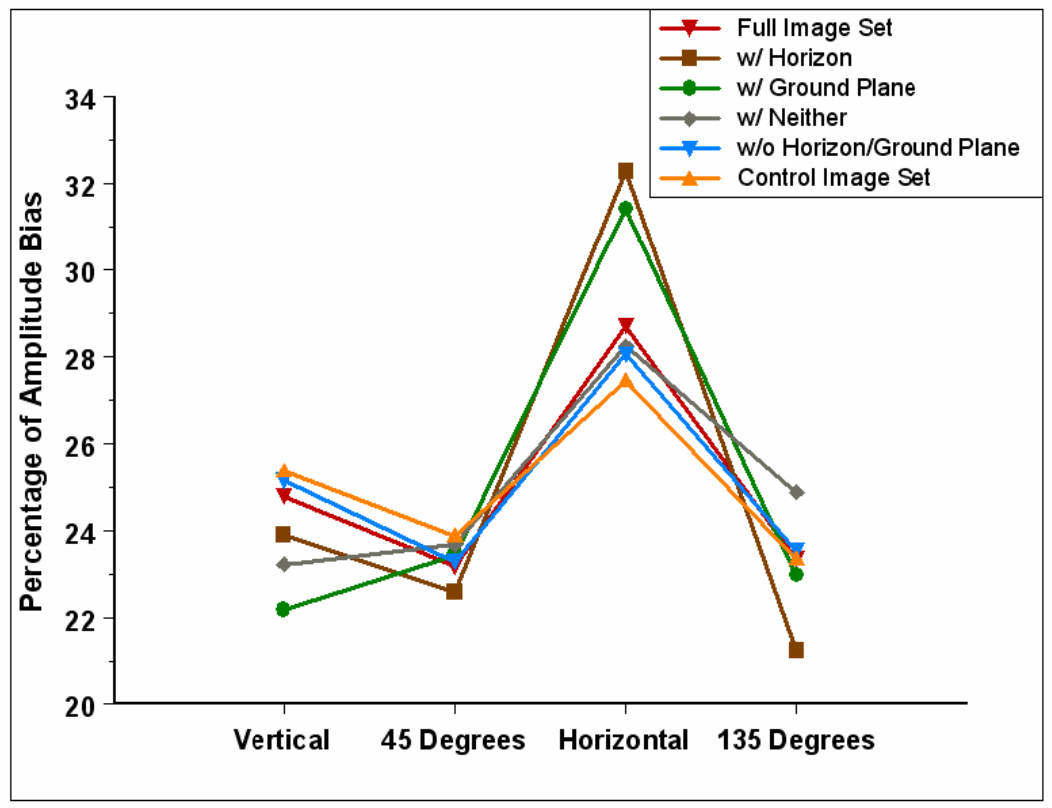

Figure 6. Using frequency analysis, each category from a 231 image data set shows a preponderance of horizontal content (Hansen \& Essock, 2004).

Studies have applied the above theories, using natural scene and broadband noise stimuli. Maloney and Clifford (2015) explored adaptive gain control in the visual cortex, theorizing that when signal is strong, resources could be diverted to what is unusual in the 
environment. Their experiments indeed showed that when image contrast increased, there is a switch from greatest BOLD signal in V1 at cardinals (most at vertical) to obliques. They found the same, though reduced, pattern in V2 and V3 as well as a constant large vertical signal in hV4. Girschik et al., (2011) showed that there is a greater orientation bias during high-noise conditions, as orientations are more likely to be judged to be closer to the nearest cardinal. They also used the Bayesian encoder-decoder model to calculate participants' priors and found that they matched that of the probability of orientation content in the natural environment, with peaks at cardinal orientations. Electrical multi-unit activity measured in ferret V1 revealed that the "internal model", or bias of the visual cortex shown from spontaneous activity, matched that of the evoked activity during natural scenes, and that this match became more similar with age, and was not found using grating stimuli (Berkes, Orbán, Lengyel \& Fiser, 2011). Again, our natural environment has a profound effect on the way our cortex encodes and interprets stimuli.

\section{Visual Reference Frames}

A visual reference frame is a coordinate system that can be used to define position, orientation, and motion (Lathan, Wang \& Clément, 2000). Therefore, by putting different reference frames in conflict, one can determine how much each of the aforementioned parameters' effects follow retinal, visual, and gravitational coordinates, which helps to determine where in the visual system the processing is taking place. Frames of reference that can be used to represent orientation in vision are: retinocentric, 
egocentric, geocentric, and patterncentric (Wade, 1992; Luyat \& Gentaz 2002; Corbett \& Carrasco, 2011).

The retinocentric (or retinal) reference frame is defined in terms of polar coordinates, with the fovea set as the origin and the retinal meridian as a cardinal axis. Unfortunately, this reference frame has no way of distinguishing between object and eye orientation; that is, if one relied only on this frame for orientation information there would be no orientation constancy and objects would appear to move and change shape during head tilt (Banks \& Stolarz, 1975). The retinocentric frame isn't only used by cells in the retina, however, as retinocentric coordinates are still retained at an early cortical level. Retinocentric signals from each eye are combined to produce the egocentric frame of reference. Here, orientation is signaled relative to head/body coordinates, and to parse apart these two, one can manipulate body tilt separately from head tilt.

Head tilt invokes an otolithic-ocular reflex causing the eyes to rotate in the opposite direction of the head-roll by about $10 \%$ of the total tilt, known as ocular counterroll (Luyat \& Gentaz 2002; Tarnutzer, Bockisch \& Straumann 2009; Tarnutzer, Fernando, Kheradmand, Lasker \& Zee, 2012). The otolith organs, the saccule and utricle, are part of the vestibular labyrinth, which is in the inner ear (Bear, Connors \& Paradiso, 2007). Both organs contain a macula, which is a small elongated structure oriented vertically in the saccule and horizontally in the utricle. The otoliths themselves are actually calcium carbonate crystals that form the outermost layer of the macula, and that are attached to hair cells by a gelatinous membrane. When head tilt occurs, both gravitational forces and acceleration move the otoliths, which in turn pull on the hair cells via the gelatinous membrane. The kinocilium is the tallest cilium within each hair cell, and it is responsible 
for signaling this mechanical stimulation. When the hair cells are pulled toward their kinocilium, they depolarize and send an excitatory signal, while being pulled in the other direction hyperpolarizes the hair cell. Because of this, movement is only sensed when it is parallel to the surface of the macula; other directions of tilt barely cause any response in the hair cells (Bear et al, 2007). In each macula, there also exists the striola, which divides the membrane evenly and in a way such that hair cells on one side of the striola have their kinocilium facing the opposite direction of the hair cells on the other side. This allows coding of the magnitude of head tilt. Signals from the otolith organs are transmitted via the vestibular nerve, which forms the vestibulocochlear nerve (cranial nerve VIII) along with the auditory nerve. While orientation constancy suggests that there must be compensatory units for head tilt, it was not thought that they appeared as early as V1. However, recently it has been shown that there are head-centered cells in rhesus monkey V1 that use eye position information to at least partially compensate for ocular counter-roll (Daddaoua, Dicke \& Their, 2014).

The geocentric, or gravitational, frame of reference incorporates signals from both the otolith organs of the vestibular system and from touch receptors (mechanoreceptors) that are sensitive to the pressure of body weight on a surface (Wade, 1992; Jenkin, Dyde, Jenkin, Howard \& Harris, 2003; though note that the vestibular signal for torsion may also incorporate signals from the semicircular canals, Snyder, 1999.). There are also smaller contributions to this reference frame from neck and trunk proprioceptors. When studying the gravitational reference frame, many authors measure the Subjective Visual Vertical (SVV), by asking subjects to adjust a line to orient it vertically, or indicate which direction is "up" (Luyat \& Gentaz, 2002; Tarnutzer, Bockisch \& Straumann, 2009). 
Therefore, SVV is different from the true physical vertical because it is influenced by the gravitational reference frame. SVV is only slightly different from physical vertical when the subject is upright (Tarnutzer et al, 2012; less than five degrees: Jenkin et al 2003), which suggests that the brain may be optimized for estimating cardinals even in the absence of a visual background. However, with head tilt, the SVV varies from physical vertical by a greater magnitude. This is important because sometimes during head tilt conditions the experimental results don't follow gravitational coordinates exactly, but do match up when SVV is used as the vertical coordinate (though McIntyre, Lipshits, Zaoui, Berthoz \& Gurfinkel, 2001, found no evidence for this). While ocular counter roll and SVV may both rely on a common otolith signal, SVV switches from over-compensation to under-compensation when the body is tilted to about 60 degrees, while ocular counter roll remains a small proportion of the tilt, slowly increasing all the way to a body tilt of 90 degrees (Tarnutzer et al, 2009).

The patterncentric (environmental) reference frame is used when a variety of orientations are simultaneously present in the environment (Wade, 1992). Extrinsic patterncentric cues are those that establish a reference to which other orientations can be assigned. That is, it describes relational orientations or objects within a display. Orientation illusions are usually instances of patterncentric interactions because the presence of one orientation influences the perception of another orientation. For example, the rod and frame effect occurs when tilting a frame that surrounds an oriented line causes a visual perceptual tilt of the line in the opposite direction of the tilt of the frame, (see Figure 7; Ebenholtz \& Benzschawel, 1977). Intrinsic patterncentric cues are those that are used to orient oneself based on the feeling that objects have an obvious "right 
way up.” These are learned through previous visual experience and are sometimes referred to as the perceptual upright (Jenkin, Dyde, Jenkin, Zacher \& Harris, 2011). For example, tilted letters are identified significantly less often when briefly flashed, than letters presented upright (Corballis, Anuza \& Blake, 1978). The shading of an object can be used as an indicator as to the direction of "up" in a scene. This is determined from the assumption that illumination comes from above when no other cues about the illumination source are readily available. This concept is known as the Light-FromAbove Prior (Jenkin et al., 2003). Patterncentric coordinates also influence the SVV (Dyde, Jenkin \& Harris, 2006, showed a tilted image behind the stimulus; Bringoux et al 2009, used a tilted virtual environment).

I

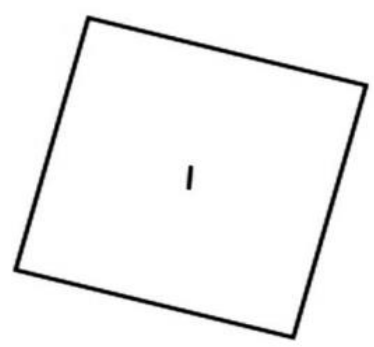

I

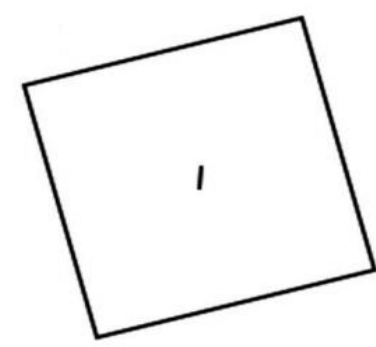

(a)

(b)

Figure 7. The rod and frame illusion. In (a) two lines of the same orientation are shown. However, when these same two lines have frames drawn around them (b) that are tilted to different directions, the rods are now perceived differently. Because the frame on the left is rotated clockwise, the rod appears tilted counterclockwise as compared to the unframed version. Vice versa is true on the right. (Corbett, Handy \& Enns, 2009).

If gravity or head tilt causes changes in results, it is very likely due to influence from the vestibular system, and so whatever causes these results must incorporate processes that occur in the visual system at or after the point at which the otolith and semicircular 
canal signal is incorporated. Note that this is different than the well-known vestibular ocular reflex (VOR), which is used to quickly stabilize the retinal image during head motion and is a quick, compensatory action. And while the pathway for the VOR is known, there are many places that incorporate both visual and vestibular information. In fact, there is no primary vestibular cortex, though there are several vestibular regions of the cortex, which makes it difficult to follow vestibular information pathways (deWaele, Baudonniere, Lepecq, Tran Ba Huy \& Vidal, 2001). Vestibular signals from cranial nerve VIII synapse on to the vestibular nuclei and the medulla (Sanders \& Gillig, 2010). The vestibular nuclei project to many cortical regions, including a dominant vestibular region adjacent to the posterior insula known as the parietal insular vestibular cortex (PIVC). This area has been found in the macaque, the squirrel monkey, and human (Guldin \& Grüsser, 1998). Though it is not the only vestibular region, it receives feedforward information from other, smaller vestibular regions as well as visual input, and also is thought to be responsible for the perception of the 'head in space'. It has also been found that the right cortical grey matter of the posterior insula is positively correlated with deviations from SVV (Dieterich \& Brandt, 2015).

The idea of a broader vestibular network is also supported by BOLD responses to vestibular nerve stimulation in the rat, (Rancz, Moya, Drawitsch, Brichta, Canals \& Margrie, 2015). Though in particular, these authors found a large response in the thalamus. In fact, large tilts of SVV have been found in patients who have lesions of the vestibular thalamus (Dieterich \& Brandt, 2015). In 2010, Barra and colleagues found that the posterolateral thalamus plays a necessary role in verticality, or orienting one's self relative to gravity. The ventral posterolateral nucleus of the thalamus projects to the 
somatosensory cortex in the parietal lobe, which sends feedback to the occipital lobe. Using electroencephalography (EEG) to measure evoked potentials, visual vertical judgements elicited brain activity in the right lateral temporo-occipital cortex; later brain activity occurred in both this area and the parieto-occipital cortex (Lopez, Mercier, Halje \& Blanke, 2011). This timing difference is thought to show the change from early stimulus form processing to later processing involving the position of a stimulus in relation to oneself as well as the position of oneself while taking gravity into consideration.

There are even still other regions involved in the vestibular cortical network and could send vestibular information to the visual cortex. The inferior parietal cortex receives vestibular afferents and is a multisensory area that includes processing of spatial orientation (Brandt, Dieterich \& Daneck, 1994). In an electrical cortical stimulation study (Blanke, Perrig, Thut, Landis \& Seeck, 2000), perception of head and body roll was induced when stimulating the intraparietal sulcus of an epileptic patient. Another study (Kheradmand, Lasker \& Zee, 2015) reported opposite-of-head-tilt judgements of SVV during TMS of the supramarginal gyrus. With this abundance of information, it is hard to say whether vestibular signals are sent to the occipital lobe from the PIVC, through the thalamus, or feedback signals from other cortices are sent back to earlier areas in the visual cortex. One must also consider that $\mathrm{V} 1$ receives more feedback information than it sends as feedforward (Haslinger et al 2012; Petro, Vizioli \& Muckli, 2014), meaning that it may be more likely to receive this vestibular information as feedback. However, as mentioned above, as the class 2 oblique effect could occur in a multisensory area, vestibular input to these areas could also be considered. 
One issue with studying head tilt only is that the majority of the time the effects of gravity are present, and so are confounded with the effects of head tilt. Because of this a common technique that can be used to discount gravity is to have subjects view stimuli while supine (Wade, 1970; Dyde, Jenkin \& Harris, 2006). Wade (1970) found that when subjects were seated upright with heads tilted, the visual head axis (visual alignment to the plane of symmetry of the head) was underestimated. However, when subjects were supine with heads tilted, most showed no effect of head tilt while some overestimated the visual head axis (though only slightly). Therefore, there actually may not be a difference in visual perception when both otolith and neck stimulation are present versus when neck stimulation alone is present. Other authors have been able to conduct experiments under zero gravity conditions, which seem to show clearer results (McIntyre et al, 2001; Jenkin et al, 2005, 2011). McIntyre and colleagues found that preference for cardinal orientations decreased during body tilt under normal gravity conditions, but was maintained during microgravity. They conclude that while head/body and gravitational reference frames are used to process orientation information under normal circumstances, the egocentric axis is used when gravity information is unavailable (also shown by Leone et al (1995), who found that the oblique effect persisted in microgravity). Jenkin et al (2005) studied the patterncentric frame in microgravity by presenting photographs of nature behind their stimuli and varying the orientation of these photos. While the patterncentric frame was incorporated in normal gravity, all coordinates aligned with the egocentric frame no matter the orientation of the photos in microgravity. Jenkin et al (2011) used static and dynamic displays to reinforce the patterncentric frame. Although the displays marginally influenced the direction of the stimulus, the findings were not 
significant and the egocentric frame remained dominant. Therefore, even when the gravitational reference frame is not present, the egocentric frame takes over and oblique effects are still present.

Tasks producing the Class 1 oblique effect follow retinal coordinates. That is, the effect will change with head tilt (Banks \& Solarz, 1975; Frost \& Kaminar, 1975; Corwin, Moskowitz-Cook \& Green; Chen \& Levi, 1996; Corbett \& Carrasco, 2011). For example, in terms of gravitationally-defined coordinates, participants will show greatest sensitivity to simple stimuli that are oriented $45^{\circ}$ and $135^{\circ}$ degrees when their heads are tilted $45^{\circ}$. This has been shown with psychophysical tasks involving contrast sensitivity, and parallelism and perpendicularity discrimination. Frost and Kaminar (1975) also showed that VEP amplitudes are greater for cardinal orientations as defined by retinal coordinates. However, some researchers have shown that the oblique effect doesn't shift one-hundred percent with head tilt, that is, it does not wholly follow retinal coordinates. The Müller, or "E-effect", occurs during head rotations of up to about 60 degrees from vertical. It results in the subject perceiving the stimulus as tilted to a lesser degree than it actually is tilted, due to overestimation of body/head tilt (Luyat \& Gentaz, 2002). Opposite of this is the Aubert, or "A-affect", where body/head tilt is underestimated when it is greater than 60 degrees, so that perception of stimulus orientation is biased in the direction of tilt. Still, other researchers have shown that even if the oblique effect doesn't completely match retinal coordinates during head tilt, any difference can be made up when correcting for ocular counter roll or variations in SVV (Banks \& Stolarz, 1975; Chen \& Levi, 1996). 
Class 2 oblique effect tasks follow gravitational coordinates (Attneave \& Olson, 1967; Buchanan-Smith \& Heeley, 1993; Ferrante, Gerbino \& Rock, 1995; Comerford, Javid \& Thorn, 2000). Here, thresholds show the same oblique effect pattern based on the physical, not retinal, coordinates of the stimuli. Tasks used that show this include orientation detection, orientation identification, orientation matching, clockwise and counterclockwise discrimination, the Goldmeier effect, and the Hermann grid illusion. Class 2 tasks can also be influenced by the patterncentric reference frame: Luyat et al (2005) showed the oblique effect decreases with tilted visual contextual cues, and Meng and Qian (2005) show that the oblique effect is influenced by both the simultaneous tilt illusion and tilt after effect. The Class 2 effect may also be influenced by what are known as phenomenological coordinates: a top-down manipulation of what is typically thought of as "up" (Attneave \& Reid, 1968; Prather, 1997). Prather himself refers to his cues (white pointer or optic flow) as visual cues, however it can be argued that they are also cognitive as participants were asked to use them to use the visual cues to define the direction of "up".

The location of the oblique effect within the visual pathway has been highly debated. One factor that has contributed to this debate is that most authors do not differentiate between Class 1 and 2, and so come to different conclusions. However, by separating these articles into two groups, dependent on whether their experimental task would elicit Class 1 or 2 effects, it is easy to see that (a) they are in agreement and (b) these two effects are truly separate. Nothing yet is known about how the horizontal effect changes with respect to head tilt. 


\section{Cortical implications}

Most studies find that the majority of early visual cortical neurons have orientation specificity that is linked to retinal coordinates. However, there are some cells that change their orientation preference based on the degree of head tilt (cat: Denney \& Adorjani, 1972; Horn, Stechler \& Hill, 1972; macaque: Sauvan \& Peterhans, 1999). This is thought to help stabilize their receptive field as defined by gravitational coordinates. And while not all of these cells completely follow gravitational coordinates, they do modify their receptive field area to at least partially compensate during tilt. The RF changes observed included alterations to both size and shape and represented a wide range of orientation preferences. Horn and colleagues (1972) found that the axes of the

RFs followed the degree of tilt most closely when a cat was rotated about its longitudinal axis. However, RF changes did not always occur immediately after head tilt, including some that took up to 35 minutes later. Some cells also overestimated the change in direction when measured directly after the cat was rotated back to its original position. These authors then lesioned the bilateral labyrinths in some cats and transected the spinal cord at the first cervical nerve in others; both of these areas signal head tilt. However, neither procedure eliminated tilt compensation effects.

Nearly all complex cells in cat area 18 are sensitive to head tilt, including cells that had no orientation tuning to cells with very narrow orientation tuning, (Reinis, Landolt, Money, Lahue \& Weiss, 1986). Changes with tilt were variable: a few RFs did not change, some compensated for head tilt, and some overcompensated. Both the length and width of RFs changed, with the position of minimum length usually coinciding with the position of minimum width. Those cells that did compensate for head tilt showed a 
wide range of original orientation preference, so that there was good representation across orientation preference. These authors also found that RFs changed with respect to tilt in labyrinthectomized animals, a procedure which removes the otoliths and semicircular canals. However, the cellular compensation in the labyrinthectomized cats were not as drastic as controls, showing an interaction between visual and vestibular systems at this level of the cortex.

Neuronal changes with respect to head tilt have also been studied in the rhesus macaque. Sauvan and Peterhans (1999) found that the majority (93\%), of cells in macaque V1 were non-compensatory for tilt; that is, they followed the retinal reference frame. However, in V2 and V3/V3A, 40\% of cells were compensatory and their preferred orientation was invariant with respect to the direction of gravity. They conclude that extra-retinal signals about the direction of gravity relative to the body/head axis are integrated with vision in the pre-striate cortex. Extending from these animal studies, there are most likely neurons in the human that are compensatory for head tilt, if not as early as $\mathrm{V} 1$, then perhaps as early as V2. 


\section{CHAPTER II}

\section{GENERAL METHODS AND ANALYSES}

There is a clear separation between the Class 1 and 2 oblique effects, though many papers do not make this distinction, and instead argue about whether the effect follows retinal or gravitational coordinates. However, when assessing methodology, it is evident that experimenters who use low level tasks (acuity and contrast threshold) find that the effect follows retinal coordinates, and those who employ higher level tasks find the effect follows gravitational coordinates. To replicate these results and make the difference between Class 1 and 2 oblique effects clear, I used separate psychophysical tests to represent each of the effects, and measured how these effects differed when reference frames were put in opposition. Also, no testing of the influence of reference frames has been done with the horizontal effect, so this is an area of research that deserves more attention. Especially as the results of these studies will provide more evidence as to where this effect occurs along the visual pathway. In the proposed experiments, to parse apart the effects of the retinocentric, patterncentric, and gravitational frames, I varied head tilt and visual world tilt. In addition, the degree to which each of these anisotropies can be influenced by top down effects was measured. Here, subjects were instructed to think of either the true physical "up" or the top of their head as the physical "up" during each of the tilt conditions. 


\section{General Methods}

\section{Participants}

All participants were students recruited by flyers placed around the University of Louisville campus and in psychology classes, or shown on Facebook, as approved by the IRB of the university. Visual acuity of at least 20/20, using corrective lenses if needed, was confirmed by the Snellen eye chart test. Astigmatism was ruled out using a clock dial type astigmatism chart and a contrast threshold test with oriented grating stimuli. The threshold test was used to confirm equal cardinal thresholds and equal oblique thresholds, and is the same test as the one described below under Experiment 1: Class 1 Oblique Effect.

\section{Materials}

In order to determine whether all of the above effects are visually defined by the retinocentric or gravitational frame of reference, participants performed tasks with their heads upright or tilted $45^{\circ}$ to the right. In order to achieve this, participants used a chin rest and forehead strap to keep their heads steady and at the same height as the stimuli (see Figure 11). During the $45^{\circ}$ tilt, a cushion was provided for comfort and to further hold the head in place.

To vary the patterncentric frame of reference, prisms were used. The environment was either unchanged (though still viewed through prims) or tilted $45^{\circ}$. In both of these conditions, room lights were left on so that the subject could observe that the environment was either upright or tilted. Phenomenological coordinates were also varied by instructing the participant to make sure he or she is thinking of a certain direction as "up". 


\section{General Analyses}

The horizontal effect was measured using broadband stimuli, in a matched contrast task. To measure oblique Class 1 and 2 effects, a contrast threshold task and a reaction time task were used, respectively. The key factor was to assess how these measures differed across four orientations $\left(0^{\circ}, 45^{\circ}, 90^{\circ}\right.$, and $\left.135^{\circ}\right)$ for the horizontal effect, and between the cardinal and oblique orientations for the Class 1 and 2 oblique effects. Analyses measured if the expected orientation effects were elicited, and if the different conditions caused any change in the strength and/or pattern of these effects. To measure each effect, participants completed the above tasks in four conditions. To assess retinal versus gravitational coordinates, head upright was compared to $45^{\circ}$ head tilt. To assess retinal versus patterncentric effects, head upright with no dove prisms was compared to head upright with dove prisms tilting the visual image $45^{\circ}$. To assess gravitational versus patterncentric effects, head upright with prisms tilting the visual image $45^{\circ}$ was compared to $45^{\circ}$ head tilt with prisms tilting the image $45^{\circ}$. To assess any phenomenological effects, $45^{\circ}$ head tilt with no instruction was compared to $45^{\circ}$ head tilt with the instruction to think of the top of the head as "up" or vertical. A table summarizing all of the conditions and comparisons, including sample size requirements is available for reference in Appendix 1. 


\section{CHAPTER III}

\section{EXPERIMENT 1: CLASS 1 OBLIQUE EFFECT}

\section{Experiment 1: Visual Reference frame defining the Class 1 Oblique Effect}

Hypothesis. The Class 1 oblique effect will follow retinal coordinates.

Aim. To better characterize the Class 1 oblique effect by putting retinal and gravitational reference frames into conflict as well as test the influence of both patterncentric and phenomenological coordinates.

Rationale. In the reference-frame literature, the majority of authors combine both classes into one oblique effect. This leads to conflicting opinions as to whether this cumulative oblique effect is defined by the retinotopic or gravitational reference frame. These experiments will help add to the idea that there are in fact two separate oblique effects that have the same anisotropic pattern, but stem from different processes in different places along the visual stream. The Class 1 effect occurs in contrast threshold and acuity tasks, as reviewed earlier, is proposed to be due to population differences of complex cells in V1 and nearby.

Procedure. The Class 1 oblique effect was measured using a two interval forcedchoice contrast threshold task. Threshold is defined as $82 \%$ correct using a 40 -trial QUEST procedure. Participants were asked to fixate on a small ring in the center of the screen, with a width of two pixels and an outer diameter of approximately 0.13 degrees, at a viewing distance of $1.92 \mathrm{~m}$. It appeared before each trial and during the $500 \mathrm{~ms}$ inter- 
stimulus-interval (ISI). The test stimulus was an $8 \mathrm{cpd}$ Gabor patch oriented at $0^{\circ}, 45^{\circ}$, $90^{\circ}$, or $135^{\circ}$ with a visual angle of one degree at full-width half-height. The interval containing the test is randomly selected each trial. Participants were instructed to press 1 or 2 on the keyboard after each trial to indicate whether they perceived the stimulus in the first or second interval (see Figure 8). Each run only contained one orientation, and so the participant completes the paradigm four times. In each block the four orientations were presented in a random order. MatLab and the Psychophysics Toolbox extension were used to implement all experiments and to create and present the stimuli. Head tilt orientations were upright and tilted $45^{\circ}$ to the right using a head-tilt apparatus (see Figure 9).

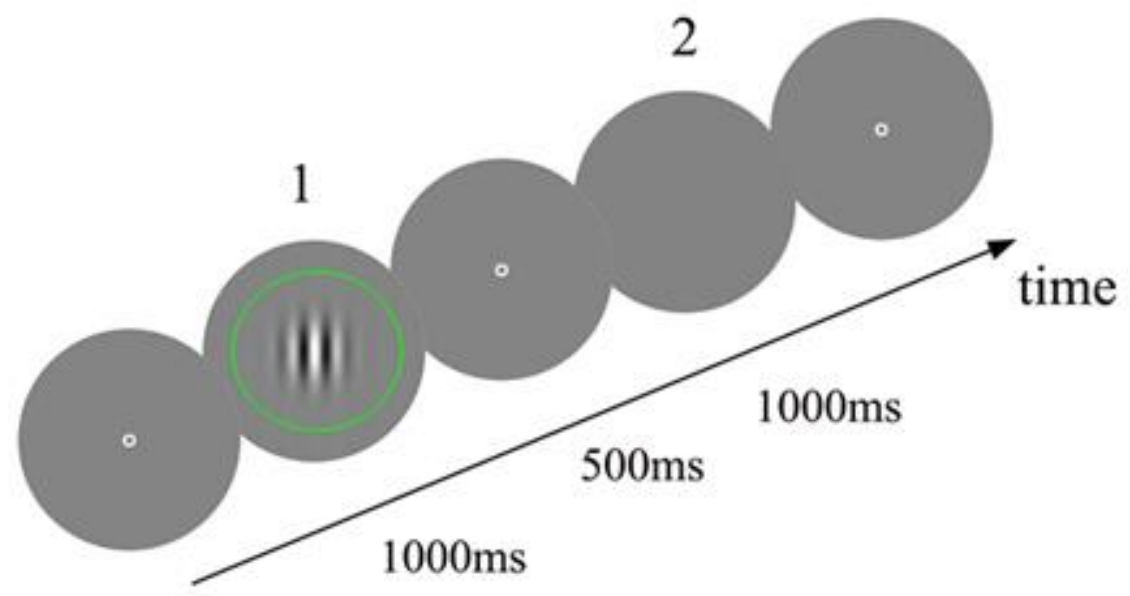

Figure 8. Example trial for the Experiment 1 paradigm that was used to study the Class 1 oblique effect. Each interval is shown for $1000 \mathrm{~ms}$ with an ISI of 500ms. Here, the stimulus appears in the first interval, so the participant would press " 1 " on the keyboard at the end of the trial. 


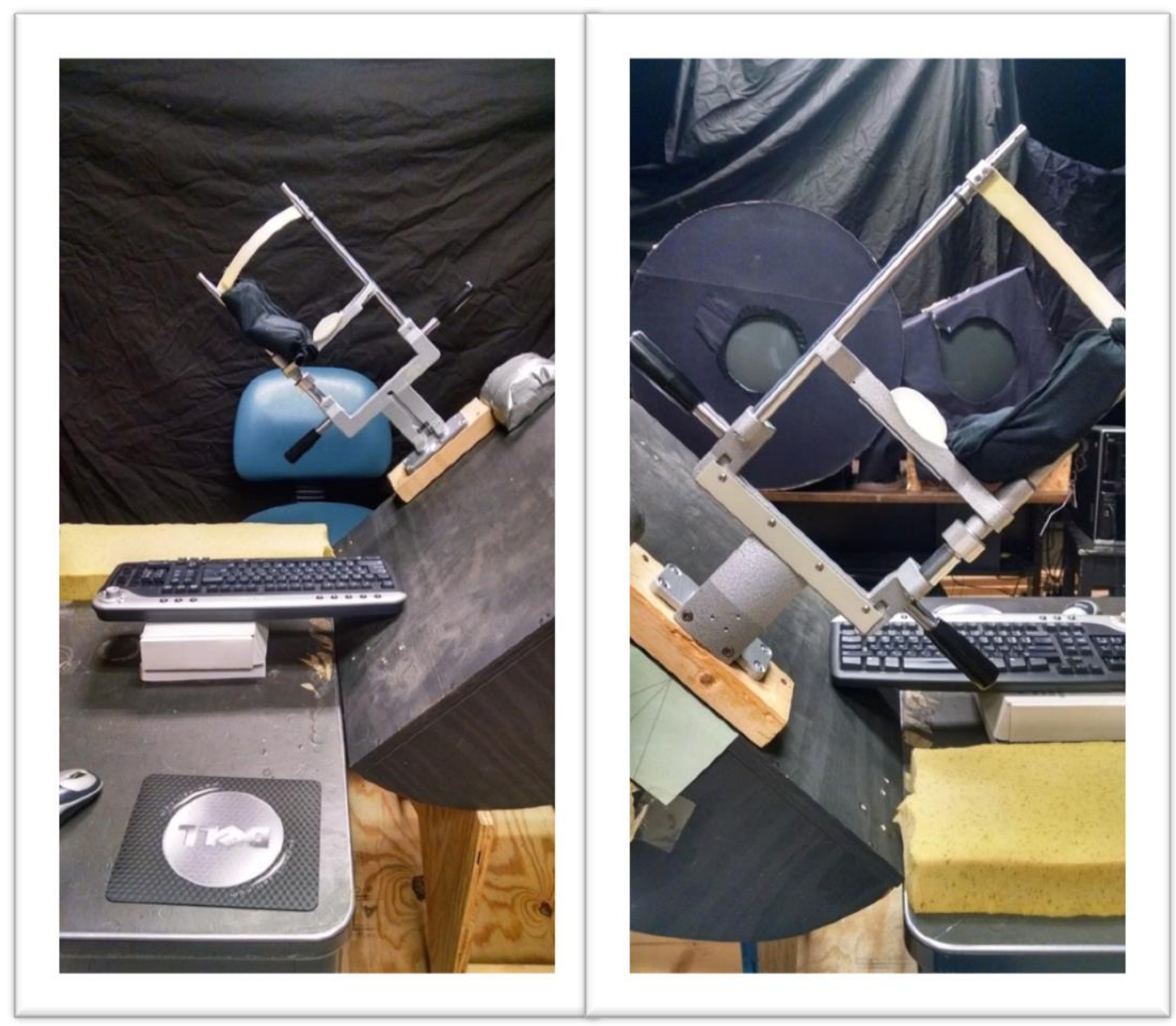

Figure 9. Head tilt apparatus with chin rest, forehead rest, and head and arm cushions. Right: view from participant's perspective.

The stimuli were displayed on a monochrome M21LMAX Image Systems, Inc. CRT monitor (white P104 phosphor). A Vision Research Graphics, Inc. grayscale expander was used in order to present a linear luminance range with a resolution of more than $2^{12}$ luminance levels. Mean luminance of the monitor was $30 \mathrm{~cd} / \mathrm{m}^{2}$ with a refresh rate of $200 \mathrm{~Hz}$, and a resolution of 800 by 600 pixels. Calibration and photometric measurements were made with an IL-1700 radiometer (International Light, Peabody, MA). All monitors had a circular annulus attached of $27^{\circ}$ in diameter with a $5^{\circ}$ aperture through which stimuli are viewed to ensure no influence of monitor edges. 
Analyses. Previous experiments using this paradigm have shown that participants generally need to complete the task five to seven times in order to minimize variability; these repetitions were averaged. Differences across orientations were analyzed using four repeated-measures, within-factors $2 \times 2$ ANOVAs. The key was to assess how threshold differs between the cardinal and oblique orientations with: head upright compared to $45^{\circ}$ head tilt, head upright with no prism goggles compared to head upright with prism goggles tilting the visual image $45^{\circ}$, head upright with prism goggles tilting the visual image $45^{\circ}$ compared to $45^{\circ}$ head tilt with prism goggles tilting the image $45^{\circ}$, and $45^{\circ}$ head tilt with no instruction compared to $45^{\circ}$ head tilt with the instruction to think of the top of the head as "up" or vertical. Based on previous screening data, a power analysis showed that 8 participants were needed for the Class 1 oblique effect task based on $\alpha=$ $0.05, \beta=0.95$, and an effect size of $f=1.4$.

Results. As proposed, each comparison was completed using a 2x2 within-factors ANOVA. One participant's data was excluded as it was found to be more than three standard deviations above the mean. Therefore, scene upright versus scene tilt and the top-down cognition analyses include seven participants, while the remainder of conditions includes the proposed eight.

First, the head upright condition was compared to $45^{\circ}$ head tilt. This allowed us to look at the influence of head tilt and stimulus orientation on contrast threshold of the grating stimuli: thresholds of cardinally-oriented and obliquely-oriented stimuli with no head tilt, were compared to threshold of cardinally-oriented and obliquely-oriented stimuli during $45^{\circ}$ head tilt (see Table 1). The interaction between head tilt and orientation was found to be significant, $F(1,7)=11.58, p<0.05, \eta_{p}^{2}=0.62$ (see Figure 
10). There was also a significant main effect of head tilt, $F(1,7)=6.60, p<0.05, \eta_{p}^{2}=$ 0.49 . There was no significant main effect of orientation, $F(1,7)=2.843, p>0.05, \eta_{p}^{2}=$ 0.29. These results show that with head upright, the cardinally-oriented stimuli had a lower contrast threshold than the obliquely-oriented stimuli, confirming the standard oblique effect pattern. Continuing to gravitationally define the stimulus orientations, head tilt caused the obliquely-oriented stimuli to have a lower contrast threshold than the cardinally-oriented stimuli. This shift of the Class 1 oblique effect with head tilt gives evidence that it is defined by retinal coordinates. The results also showed a change in contrast threshold for cardinal stimuli, while the contrast threshold for oblique stimuli remained the same, i.e. the main effect of head tilt. It could be that head tilt causes an increase in contrast threshold for cardinals, while the contrast threshold for oblique stimuli does not change. However, it is more likely that head tilt causes contrast thresholds to increase in general due to the uncommon head position.

\begin{tabular}{r|lcc} 
& & Mean & Standard deviation \\
\hline Head upright & Cardinal stimuli & 0.014 & 0.0038 \\
& Oblique stimuli & 0.020 & 0.0060 \\
& Cardinal stimuli & 0.024 & 0.0094 \\
& Oblique stimuli & 0.020 & 0.0080
\end{tabular}

Table 1. Mean and standard deviation of contrast threshold for cardinally- and obliquely-oriented stimuli during $0^{\circ}$ and $45^{\circ}$ head tilt. 


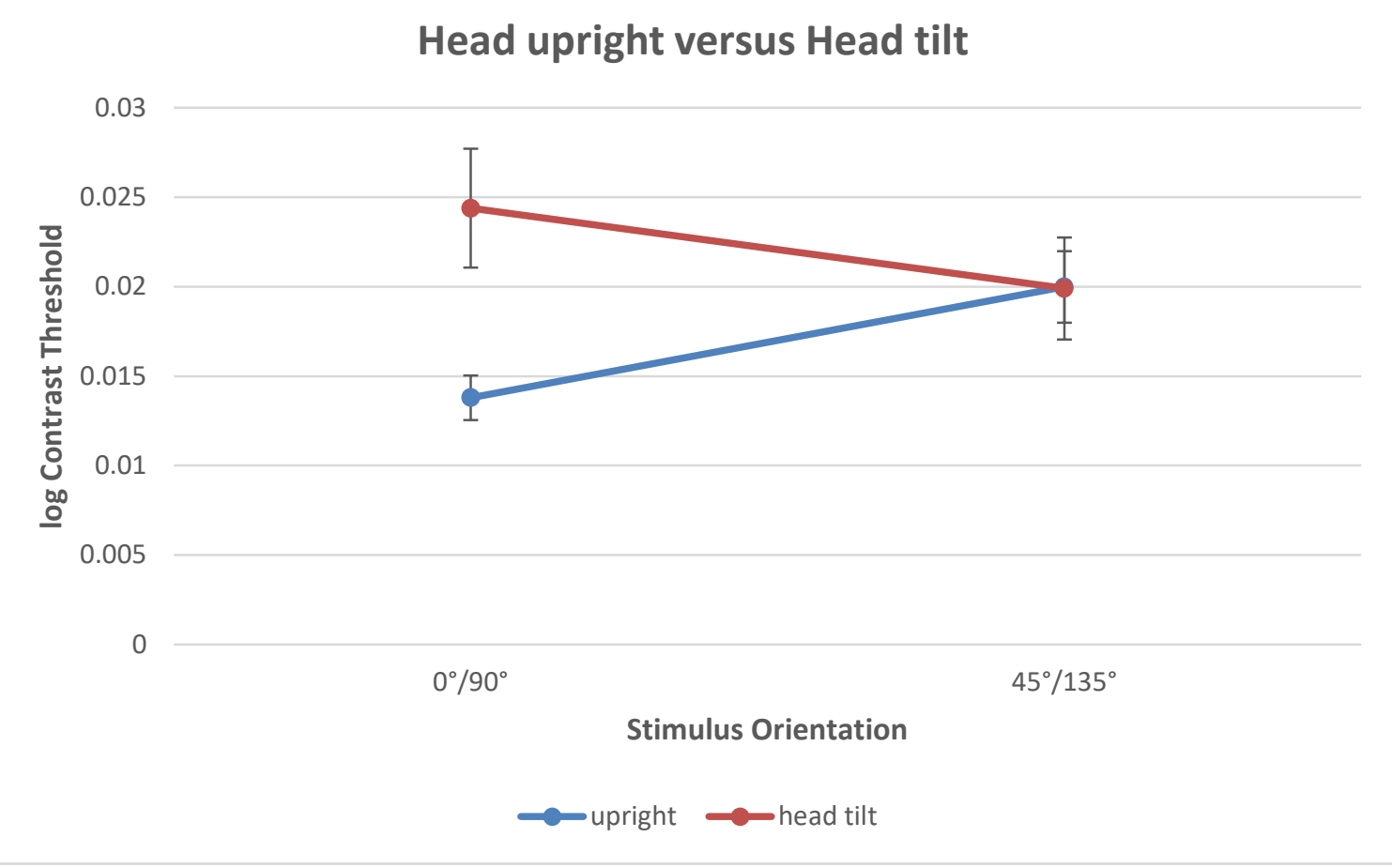

Figure 10. Contrast threshold for cardinal and oblique stimulus orientations during both $0^{\circ}$ and $45^{\circ}$ head tilt. Error bars represent standard error.

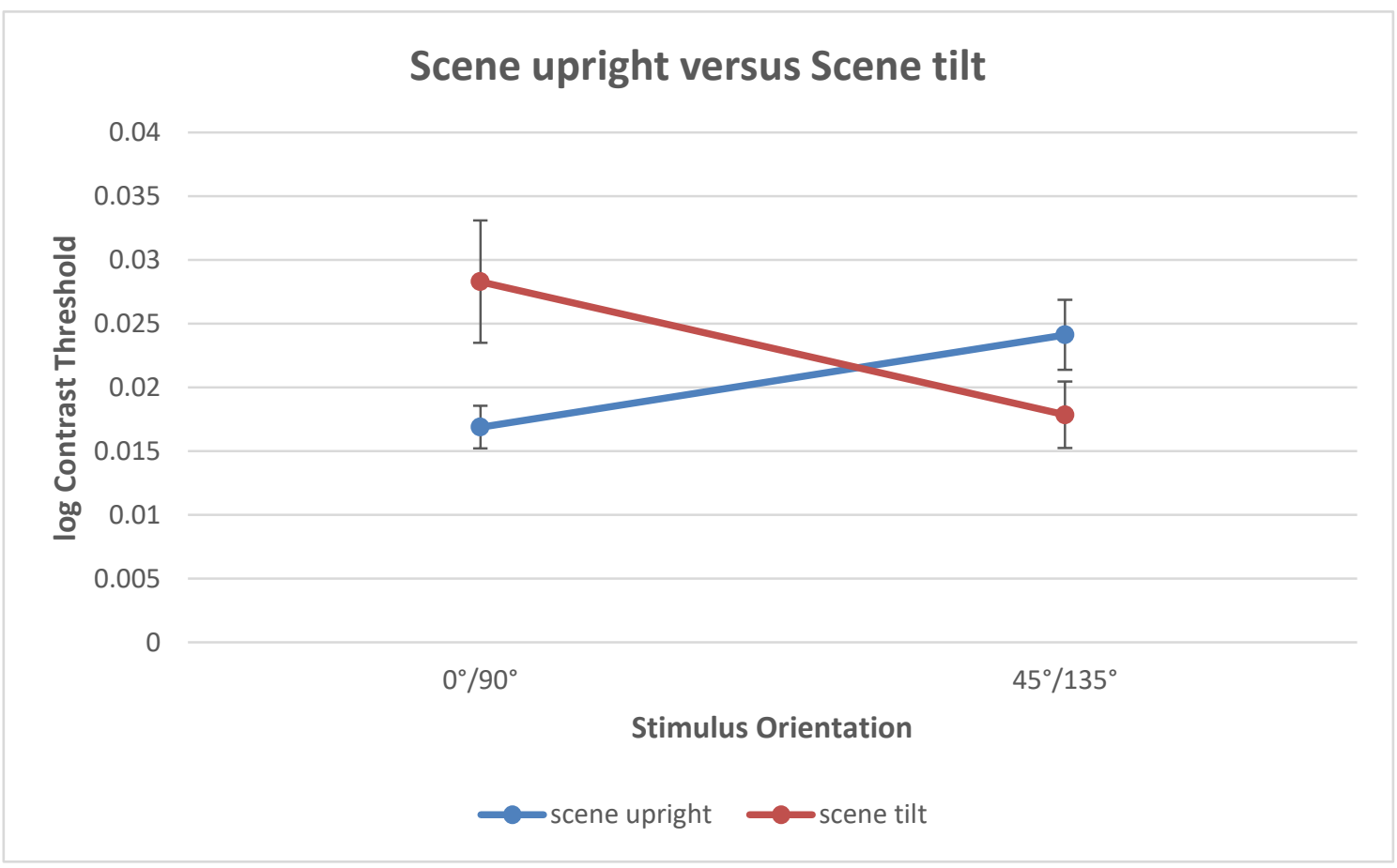

Figure 11. Contrast threshold for cardinal and oblique stimulus orientations during both $0^{\circ}$ and $45^{\circ}$ scene tilt. Error bars represent standard error. 


\begin{tabular}{r|lcc} 
Scene upright & Cardinal stimuli & 0.017 & 0.0044 \\
& Oblique stimuli & 0.024 & 0.0073 \\
& Oblique stimuli & 0.018 & 0.013 \\
& Cardinal stimuli & 0.028 & 0.0069
\end{tabular}

Table 2. Mean and standard deviation of contrast threshold for cardinally- and obliquely-oriented stimuli during $0^{\circ}$ and $45^{\circ}$ scene tilt.

The next comparison analyzed the influence of scene tilt and stimulus orientation on contrast threshold of grating stimuli (see Figure 11 and Table 2). The contrast thresholds of cardinally-oriented and obliquely-oriented stimuli with no scene tilt, were compared to the contrast thresholds of cardinally-oriented and obliquely oriented stimuli with $45^{\circ}$ scene tilt. There was a significant interaction found between scene tilt and orientation, $F(1,7)=23.29, p<0.01, \eta_{p}^{2}=0.80$. There were no significant main effects found: not for scene tilt, $F(1,7)=0.83, p>0.05, \eta_{p}^{2}=0.40$ nor for orientation, $F(1,7)=$ 2.18, $p>0.05, \eta_{p}^{2}=0.27$. These results indicate that with the scene upright, gravitationally-defined cardinal orientations have a lower contrast threshold than gravitationally-defined oblique orientations, i.e. standard oblique effect. Again, continuing to gravitationally define the stimulus orientations, scene tilt causes the obliquely oriented stimuli to have a lower contrast threshold than the cardinally oriented stimuli. The shift in contrast threshold with scene tilt could mean that the Class 1 oblique effect is changed by either the change in patterncentric coordinates or retinal coordinates. 
However, during head tilt, the patterncentric coordinates do not change, but the retinal coordinates do. Therefore, it is most likely that the change in these conditions is due to the shift in retinal coordinates.

The next experiment also compared scene upright and scene tilt, but this time during head tilt to compare head tilt and scene tilt: contrast thresholds for cardinallyoriented and obliquely-oriented stimuli with head tilt but no scene tilt, versus contrast thresholds for cardinally-oriented and obliquely-oriented stimuli with head tilt and scene tilt (see Table 3). I also expected to see the oblique effect align with retinal coordinates and so shift with scene tilt regardless of the head being tilted between these conditions. The results confirm my hypothesis (see Figure 12). There was a significant interaction between scene tilt and orientation, $F(1,7)=11.94, p<0.05, \eta_{p}^{2}=0.63$. There was no main effect for scene tilt, $F(1,7)=0.32, p>0.05, \eta_{p}^{2}=0.04$ nor for orientation, $F(1,7)=0.48, p$ $>0.05, \eta_{p}^{2}=0.06$. The results show that the contrast threshold for cardinal stimuli was higher than for oblique stimuli with scene upright with head tilt, because the head tilt caused the retinal coordinates to shift. Then, when the scene was tilted $45^{\circ}$ to the left, this caused the retinal coordinates to tilt as well and the contrast threshold for cardinal stimuli became lower than that for oblique stimuli. This shift of the Class 1 oblique effect with both head and scene tilt combine even further adds evidence that this effect is defined by retinal coordinates. 


\begin{tabular}{r|lcr} 
Head tilt and scene & Cardinal stimuli & 0.032 & 0.011 \\
Head tilt and scene & Oblique stimuli & 0.027 & 0.011 \\
tilt & Oardinal stimuli & 0.027 & 0.012 \\
& Oblique stimuli & 0.036 & 0.015
\end{tabular}

Table 3. Mean and standard deviation of contrast threshold for cardinally- and obliquely-oriented stimuli during $45^{\circ}$ head tilt for $0^{\circ}$ and $45^{\circ}$ scene tilt.

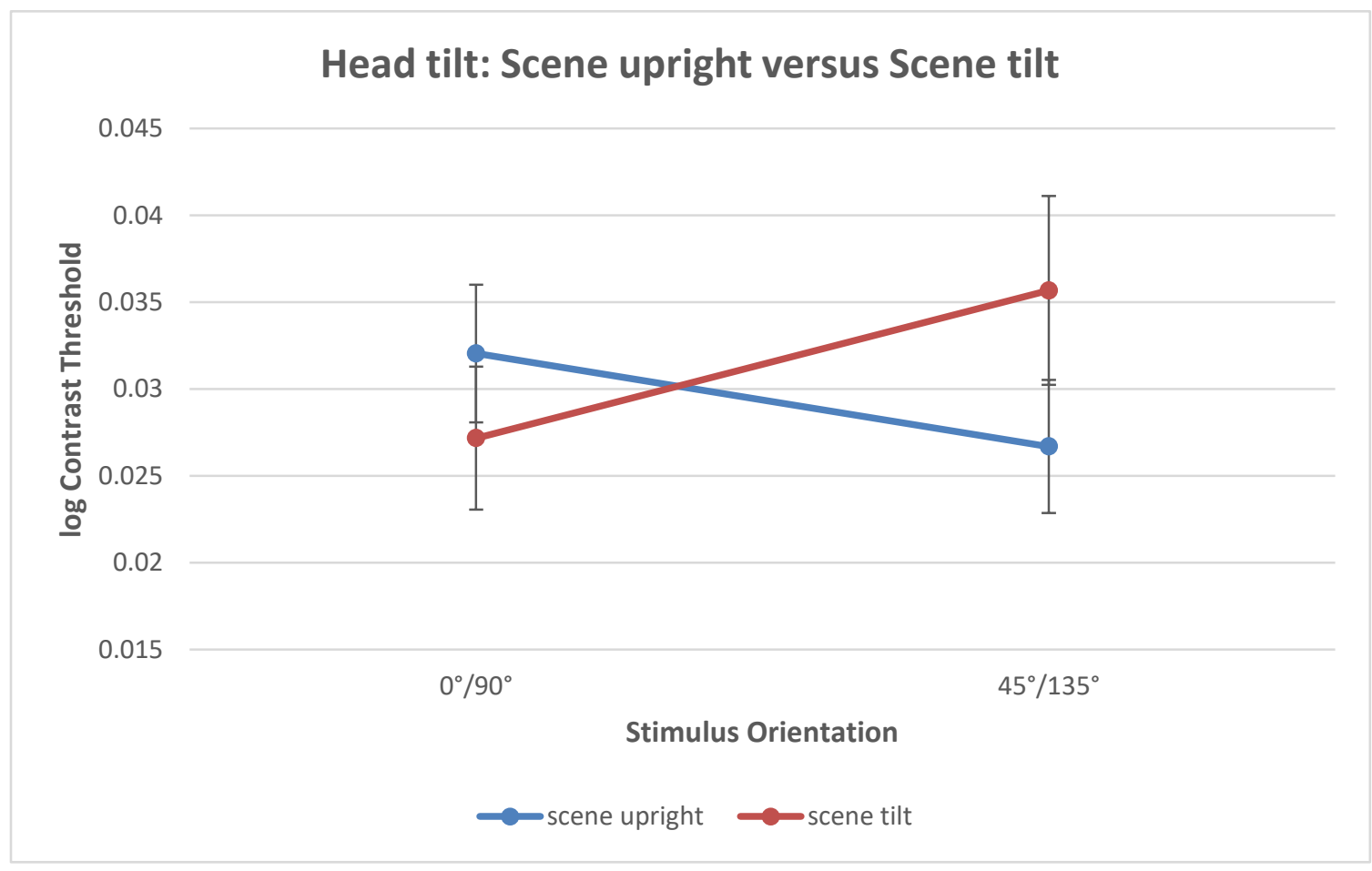

Figure 12. Contrast threshold for cardinal and oblique stimulus orientations during $45^{\circ}$ head tilt for both $0^{\circ}$ and $45^{\circ}$ scene tilt. Error bars represent standard error.

The final condition explored any top-down cognitive effect on the Class 1 oblique effect. Head tilt with no additional thoughts was compared to head tilt while participants were instructed to constantly think of the top of their head as up, or as a vertical coordinate. Here, because I am classifying the Class 1 oblique effect as a low-level visual 
effect, I hypothesized that it would not be influenced by top-down cognition and that these two conditions would look the same. The results show this to be true (see Figure 13 and Table 4) when comparing contrast thresholds of cardinal and oblique stimuli with no additional thoughts, to contrast thresholds of cardinal and oblique stimuli with a topdown cognition component. Again, all of the above conditions were measured during $45^{\circ}$ head tilt. There was no significant interaction between cognition and orientation, $F(1,7)=$ $0.25, p>0.05, \eta_{p}^{2}=0.04$ and there was no significant main effect of cognition, $F(1,7)=$ $0.09, p>0.05, \eta_{p}^{2}=0.015$. However, there was a significant main effect of orientation, $F(1,7)=41.85, p<0.01, \eta_{p}^{2}=0.88$ that shows cardinally-oriented stimuli to have a higher contrast threshold than obliquely-oriented stimuli, because of head tilt.

Mean

\begin{tabular}{r|lcr} 
No thoughts & Cardinal stimuli & 0.019 & 0.0053 \\
& Oblique stimuli & 0.016 & 0.0055 \\
Top-down cognition & Cardinal stimuli & 0.020 & 0.0063 \\
& Oblique stimuli & 0.015 & 0.0049
\end{tabular}

Standard deviation

Table 4. Mean and standard deviation of contrast threshold for cardinally- and obliquely-oriented stimuli during $45^{\circ}$ head tilt with no thought task and during instructions to think of the top of the head as up/defining vertical. 


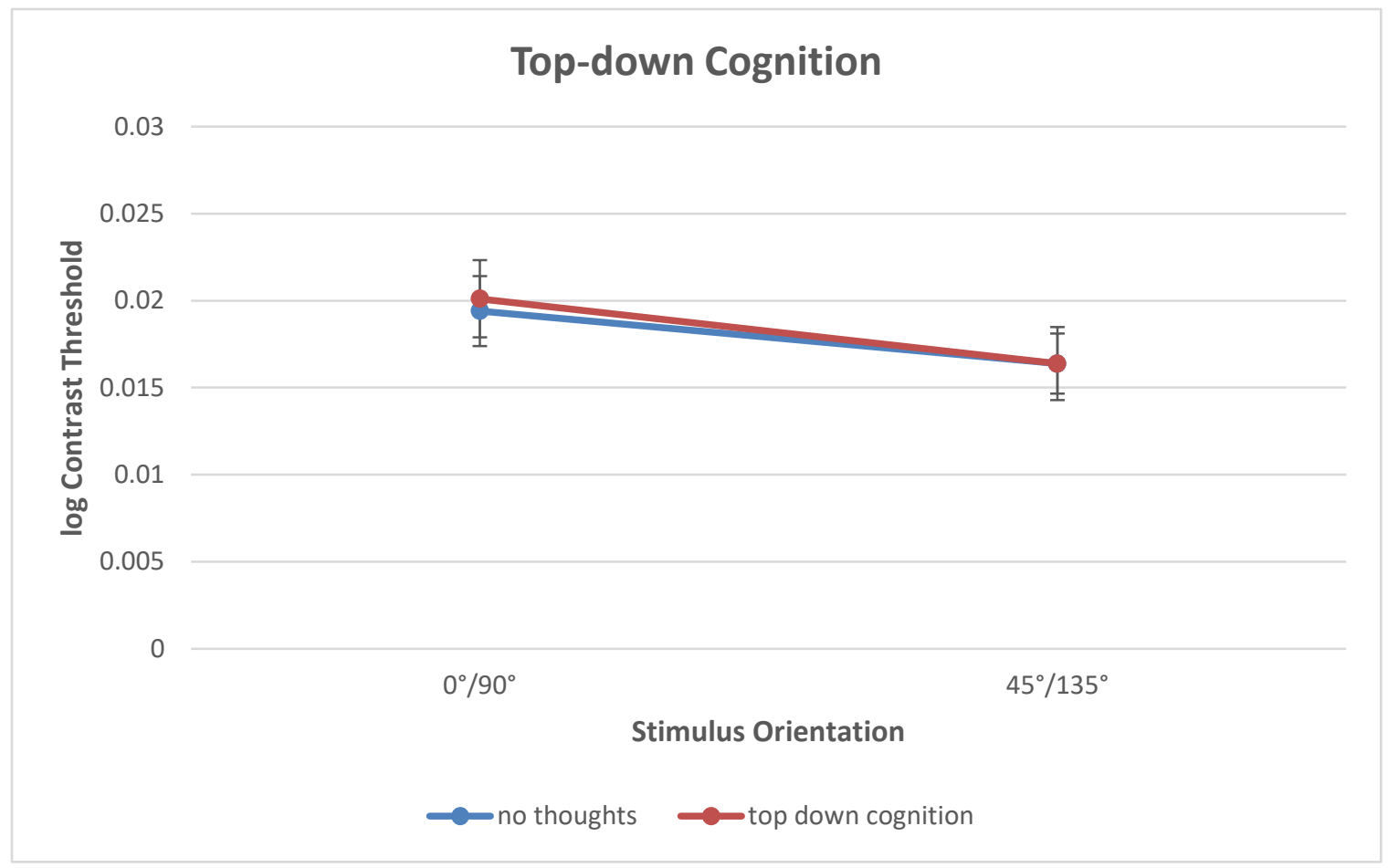

Figure 13. Contrast threshold for cardinal and oblique stimulus orientations during head tilt with no additional thoughts, and head tilt while participants were instructed to constantly think of the top of their head as up. Error bars represent standard error.

Summary. This experiment was conducted to study how the visual system defines coordinates for the Class 1 oblique effect. As mentioned in the background of the oblique effect, most studies do not differentiate between Class 1 and 2 oblique effects. However, as I argue there are two classes, and that the task and stimuli used in this experiment fits the definition of Class 1 as it measures the low-level processing of the visual system, likely in V1 or V2. Combined, all of the above data shows that the human visual system uses retinal coordinates to define orientation in the Class 1 oblique effect. With heads upright, results show the standard oblique effect where the contrast thresholds for the cardinally-oriented Gabor patches were lower than the contrast thresholds for the obliquely-oriented stimuli. When participants tilted their heads to the right $45^{\circ}$, the effect shifted along with the head tilt so that the oblique stimuli had lower contrast thresholds 
than the cardinal stimuli. As above, I am defining the stimuli by their gravitational coordinates; using retinal coordinates, the cardinal stimuli remain at lower contrast thresholds as compared to the oblique stimuli. This change with respect to head tilt shows that the visual system was using retinal coordinates to define the orientations of the stimuli.

Next, while participants viewed the stimuli through dove prisms with no scene tilt (the control), the results showed the standard oblique effect of lower contrast sensitivity for cardinally- than for obliquely-oriented stimuli. With the visual scene tilted $45^{\circ}$ to the left, the effect also tilted so that participants had lower contrast sensitivity for the oblique than the cardinal stimuli, gravitationally defined. The tilt of the oblique effect pattern could be due to a change in patterncentric coordinates, but is most likely due to the change in retinal coordinates as seen in the head tilt condition. To explore further, the head tilt + prism conditions were analyzed. When the scene remained upright (control), but head is tilted, the results show the oblique effect changes with respect to retinal coordinates so that gravitationally/patterncentrically defined, the contrast thresholds are higher for cardinal than oblique stimuli. This condition mirrors the head tilt (no prism) condition and provides further evidence that the effect is changing with retinal coordinates and not because patterncentric coordinates are changing during scene tilt. Then, during $45^{\circ}$ head tilt to the right and $45^{\circ}$ scene tilt to the left, the retinal coordinates are aligned with gravitational coordinates, while patterncentric coordinates are rotated to the left. The oblique effect again aligns itself with retinal coordinates and shows the standard pattern of lower contrast thresholds for cardinal than for oblique stimuli. 
The final condition tested to see if the Class 1 oblique effect pattern could be changed through top down cognition by changing phenomenological coordinates. While participants' heads were tilted, they were instructed to think of the top of the head as up, or defining vertical. However, because the retinal coordinates were aligned with the phenomenological coordinates it is impossible to tell if the change in phenomenological conditions had any effect. A condition with the head tilted where participants were instructed to think of gravitational "up" as up would help here. It is worth nothing that while not constantly reminding ourselves, we do still tend to consider gravitational "up" as up during head tilt, and are even likely to shift what we consider vertical the opposite direction of head tilt (E effect; Wade, 1970; 1992; Luyat \& Gentaz, 2002; Tarnutzer, Bockisch \& Straumann 2009).

The results here supporting that the human visual system defines the oblique effect pattern with respect to retinal coordinates is in agreement with the majority of previous articles studying the coordinates of the oblique effect using stimuli and tasks that can be classified as activating low-level processes. (Banks \& Solarz, 1975; Frost \& Kaminar, 1975; Corwin, Moskowitz-Cook \& Green; Chen \& Levi, 1996; Corbett \& Carrasco, 2011). The results here do not agree with papers with tasks and stimuli that can be classified as activating mid- to high-level visual processes, which find the oblique effect to be tied to gravitational coordinates (Attneave \& Olson, 1967; Buchanan-Smith \& Heeley, 1993; Ferrante, Gerbino \& Rock, 1995; Comerford, Javid \& Thorn, 2000). This is why I am arguing that there are two classes of the oblique effect. The Class1 oblique effect, which I show can be defined by retinal coordinates, and the Class 2 oblique effect, which is explored in the next chapter. 


\section{CHAPTER IV}

\section{EXPERIMENT 2: CLASS 2 OBLIQUE EFFECT}

\section{Experiment 2: Visual Reference frame defining the Class 2 Oblique Effect}

Hypothesis. The Class 2 oblique effect will follow gravitational coordinates.

Aim. To better characterize the Class 2 oblique effect by putting retinal and gravitational reference frames into conflict as well as by testing the influence of both patterncentric and phenomenological coordinates.

Rationale. As per experiment 1, these experiments further investigated the proposal that that there are in fact two separate oblique effects that have the same anisotropic pattern, but stem from different processes in different places along the visual stream. The Class 2 effect occurs in tasks that involve how orientation is phenomenologically represented. Therefore, it is influenced not only by cellular populations and gain control processes (lower level processes), but also by how we internalize orientation (higher level processes) - making up a kind of reference frame in which to help define our world and better situate ourselves in it.

Procedure. During the experiment, oriented Gabor patches were presented randomly in one of eight equally-separated locations around the fixation point (see Figure 14). The fixation point was the same as in the Class 1 oblique effect paradigm and will appear before each trial. The stimuli themselves were 0.5 degrees in diameter, with their 
centers always located 0.75 degrees from the fixation point. The patches were oriented at $0^{\circ}, 45^{\circ}, 90^{\circ}$, and $135^{\circ} \mathrm{s}$ organized pseudo-randomly so that two of each were be randomly presented within every eight trials. This was to ensure that there are not multiple trials of a single orientation, as well as to keep the participant from easily guessing the next

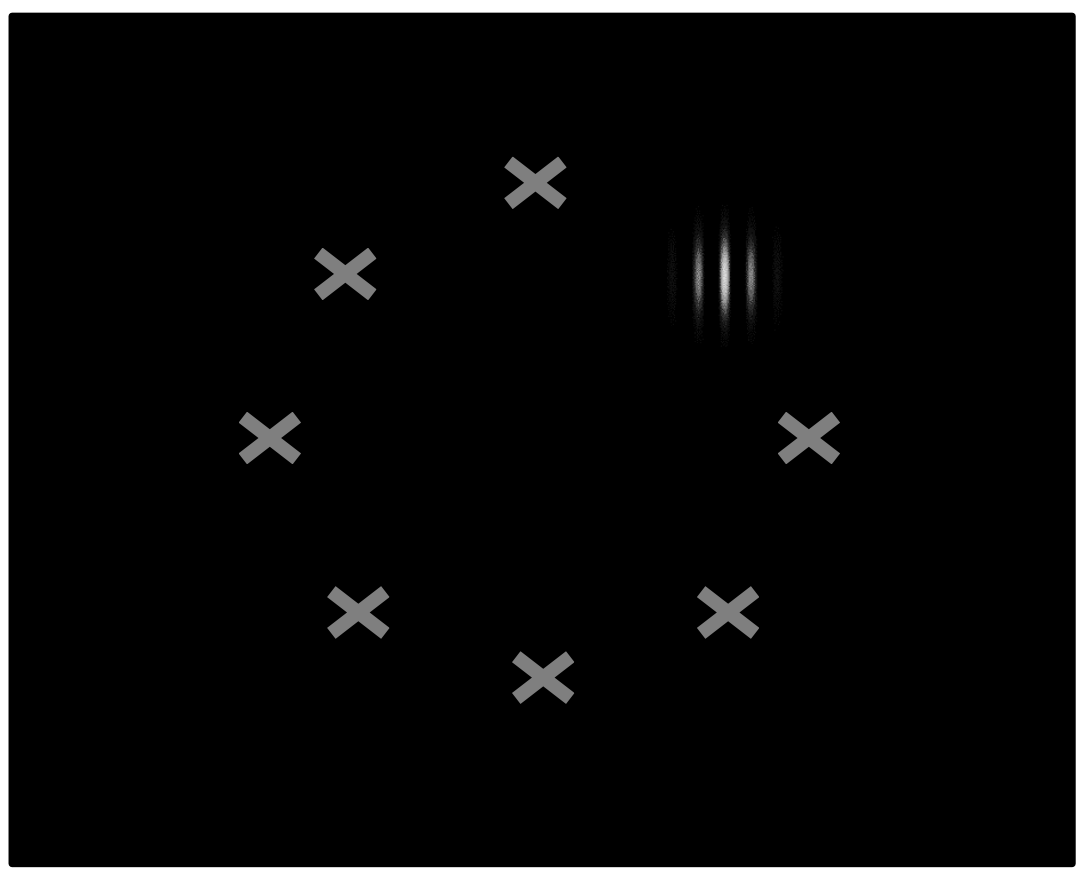

Figure 14. Example vertically-oriented stimuli at the eight possible locations in the reaction time task designed to measure the Class 2 oblique effect.

stimulus. The participant's task was to press one of four keys corresponding to the orientation of the stimulus that appears on screen, and to do so as fast as possible, and reaction time was be recorded. The keys were always 1,2,3, and 4 on the keyboard but the orientations they represented were different across participants so that finger order or procedural memory can be ruled out. The delay it takes for the computer to detect a key press was measured for the keyboard used, and an average delay of 33 milliseconds was found. These stimuli were shown using the same equipment as that of Experiment 1. 
Analyses. Repetitions of reaction time measurement were averaged for analysis of the oblique effect pattern. Outliers were removed if they were above three standard deviations from the mean. Differences across orientations were analyzed using four repeated-measures, within-factors $2 \times 2$ ANOVAs. The key was to assess how reaction time differs between the cardinal and oblique orientations with: head upright compared to $45^{\circ}$ head tilt, head upright with no prism goggles compared to head upright with prism goggles tilting the visual image $45^{\circ}$, head upright with prism goggles tilting the visual image $45^{\circ}$ compared to $45^{\circ}$ head tilt with prism goggles tilting the image $45^{\circ}$, and $45^{\circ}$ head tilt with no instruction compared to $45^{\circ}$ head tilt with the instruction to think of the top of the head as "up" or vertical. Power analysis for the Class 2 oblique effect task was calculated using the effect size of previous work by Essock (1980; experiment 2), as the proposed methodology is a close replication of that task. With $\alpha=0.05, \beta=0.95$, and an effect size of $f=0.91$, the power analysis shows that 8 participants were needed for the Class 2 oblique effect task.

Results. As proposed, each comparison was completed using a $2 \times 2$ within-factors ANOVA. No participants were excluded from analysis. To test whether the Class 2 oblique effect changes with head tilt or stays tied to gravitational coordinates, I looked at the influence of both head tilt $\left(0^{\circ}\right.$ or $\left.45^{\circ}\right)$ and stimulus orientation (cardinal or oblique) on participants' reaction time (ms) to the appearance of oriented Gabor patches. That is, cardinally- and obliquely-oriented stimuli with no head tilt, were compared to cardinallyand obliquely-oriented stimuli during head tilt (see Table 5). The results show no significant interaction between head tilt and orientation, $F(1,8)=2.69, p>0.05, \eta_{p}^{2}=$ 
0.25 and no significant main effect of head tilt, $F(1,8)=3.87, p>0.05, \eta_{p}^{2}=0.33$ (see

Figure 15). However, there was a main effect of orientation, $F(1,8)=22.52, p<0.01, \eta_{p}^{2}$ $=0.74$. These results tell us that head tilt made no difference, and so in this case the visual system is defining the Class 2 oblique effect with gravitational coordinates. The significant main effect of orientation shows the classic oblique effect of a faster reaction time to cardinally- versus obliquely-oriented stimuli.

\begin{tabular}{c|lcc}
\multicolumn{2}{c}{} & Mean & Standard deviation \\
Head upright & Cardinal stimuli & 0.70 & 0.34 \\
& Oblique stimuli & 0.83 & 0.32 \\
& Cardinal stimuli & 0.63 & 0.23 \\
& Oblique stimuli & 0.69 & 0.22
\end{tabular}

Table 5. Mean and standard deviation of reaction times for cardinally- and obliquelyoriented stimuli during $0^{\circ}$ and $45^{\circ}$ head tilt. 


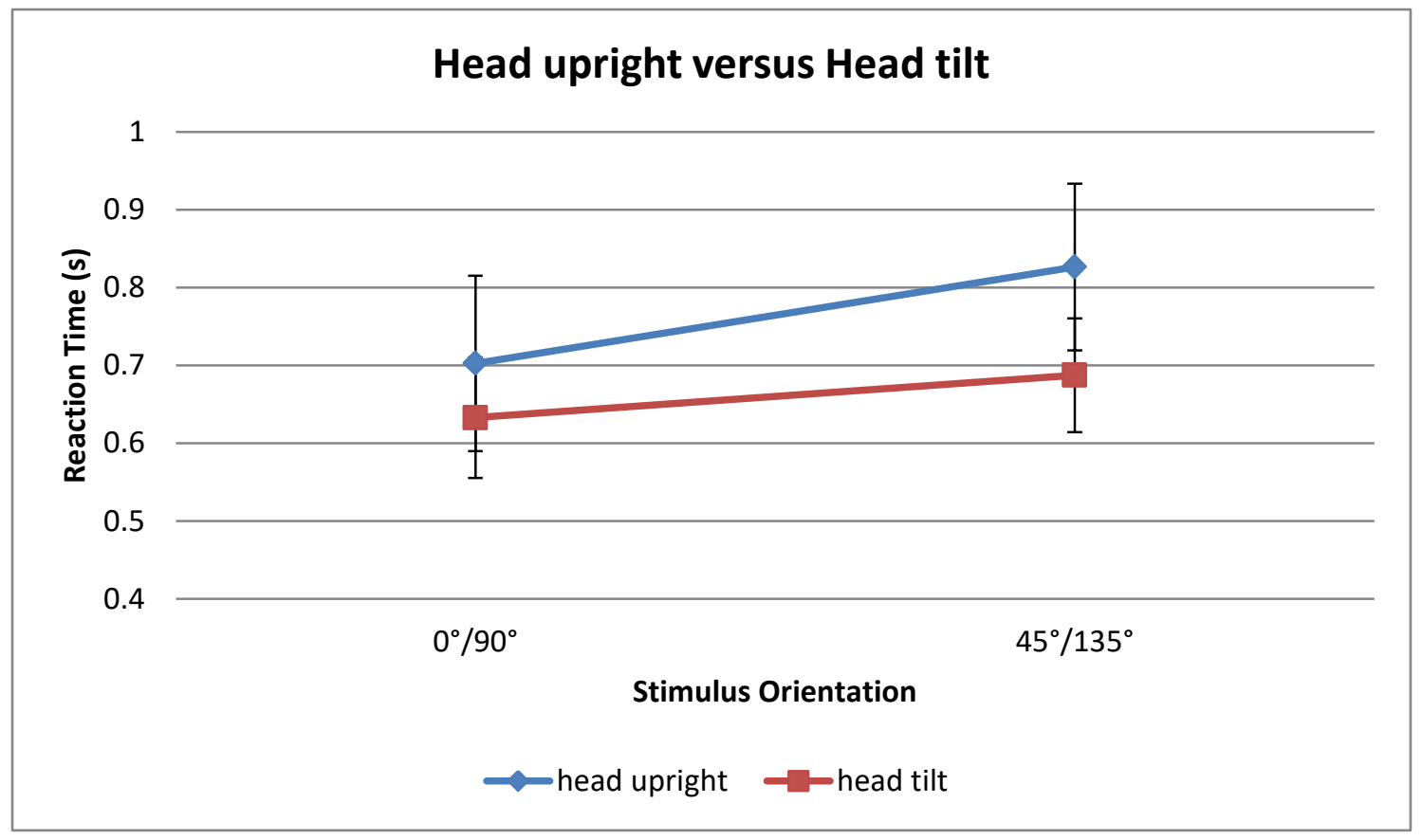

Figure 15. Reaction time data for cardinal and oblique stimulus orientations during $0^{\circ}$ and $45^{\circ}$ head tilt. Error bars represent standard error.

Next, scene upright was compared to $45^{\circ}$ scene tilt to see if the Class 2 oblique effect changes with patterncentric coordinate changes or continues to stay tied to gravitational coordinates. The results show a significant interaction between scene tilt and orientation, $F(1,8)=40.43, p<0.01, \eta_{p}^{2}=0.85$ (see Figure 16 and Table 6$)$. There is no significant main effect for either scene tilt, $F(1,8)=0.07, p>0.05, \eta_{p}^{2}=0.01$ or for orientation, $F(1,8)=3.22, p>0.05, \eta_{p}^{2}=0.32$. So here, results show the standard Class 2 oblique effect during no scene tilt, where the reaction time for the cardinally-oriented Gabor patches was faster than the reaction time for the obliquely-oriented Gabor patches. This changes with scene tilt so that the opposite is true. The change in coordinates could be due to retinal coordinate changes, however this seems unlikely when considering the 
results of the head tilt comparison. It is most likely that the change patterncentric coordinates causes the rotation of the oblique effect.

\begin{tabular}{r|lcc} 
Scene upright & Cardinal stimuli & 0.50 & 0.13 \\
& Oblique stimuli & 0.61 & 0.17 \\
& Cardinal stimuli & 0.56 & 0.20 \\
& Oblique stimuli & 0.51 & 0.17
\end{tabular}

Table 6. Mean and standard deviation of reaction times for cardinally- and obliquelyoriented stimuli during $0^{\circ}$ and $45^{\circ}$ scene tilt.

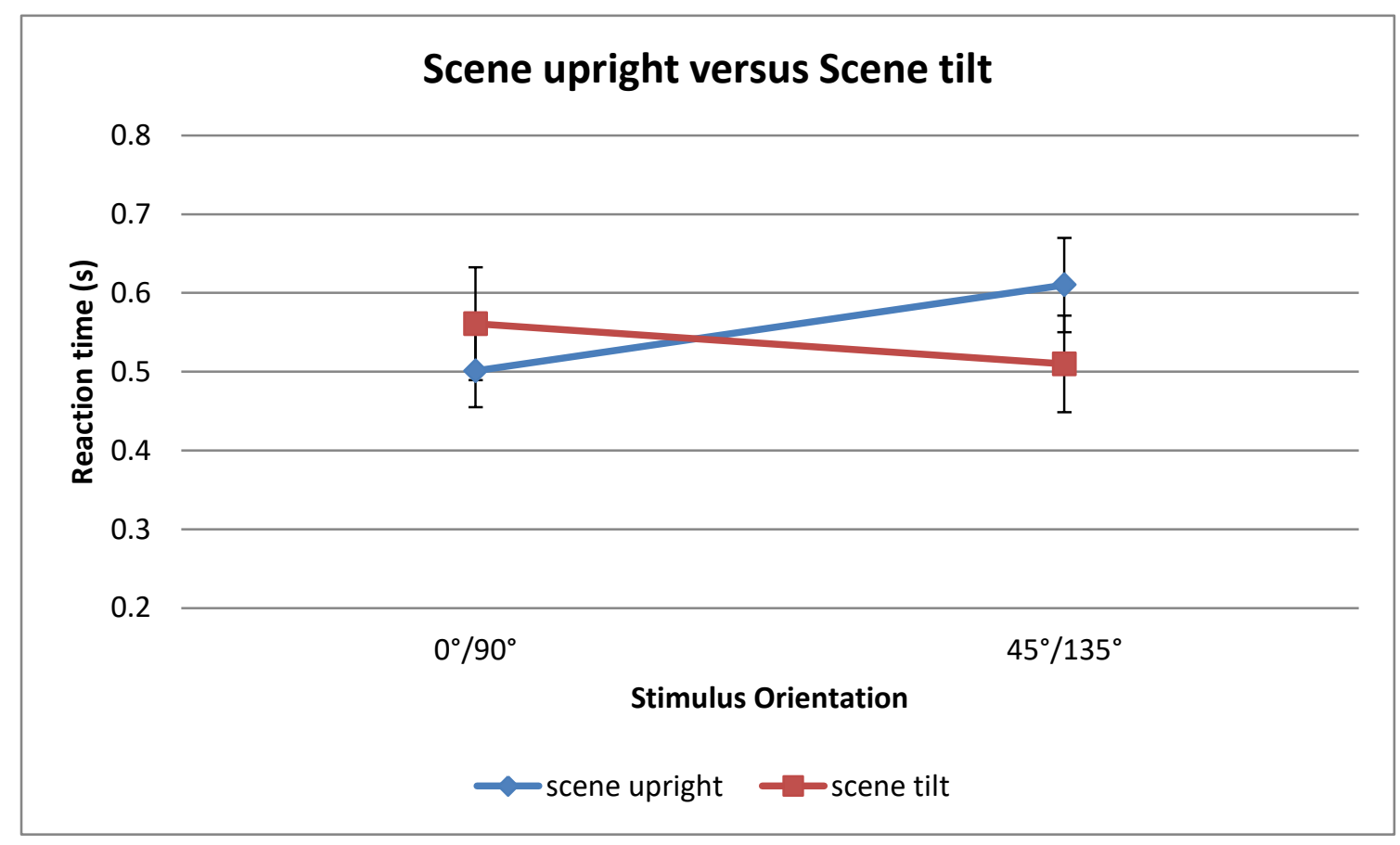

Figure 16. Reaction time data for cardinal and oblique stimulus orientations during $0^{\circ}$ and $45^{\circ}$ scene tilt. Error bars represent standard error.

To further differentiate between head tilt and scene tilt, participants were tested again during $45^{\circ}$ head tilt, but this time included scene tilt of $0^{\circ}$ or $45^{\circ}$. That is, head tilt 
and scene upright with cardinal and oblique stimuli, were compared to head tilt and scene tilt with cardinal and oblique stimuli (see Table 7). There was no significant interaction between scene tilt and stimulus orientation, $F(1,7)=2.37, p>0.05, \eta_{p}^{2}=0.25$, nor was there a significant main effect of stimulus orientation, $F(1,7)=1.72, p>0.05, \eta_{p}^{2}=0.20$. There was a significant main effect of scene tilt, $F(1,7)=20.61, p<0.01, \eta_{p}^{2}=0.75$ (see Figure 17). The head tilt and scene upright condition did not show a strong difference across stimulus orientation, which was not what I expected. The head tilt and scene tilt does show trend of decreased reaction time for obliquely-oriented stimuli, which is what would be expected as I am postulating that the effect changes with patterncentric coordinates. Along with this, there is no main effect of orientation, which would be seen if the two conditions produced the same trend, unless there were no differences across orientation. The main effect of head tilt is unsurprising, as participants were still required to categorize orientations gravitationally, which was difficult with both head and scene tilt.

Mean

\begin{tabular}{r|lcc} 
Head tilt and scene upright & Cardinal stimuli & 0.68 & 0.27 \\
& Oblique stimuli & 0.70 & 0.22 \\
Head tilt and scene tilt & Cardinal stimuli & 1.03 & 0.37 \\
& Oblique stimuli & 0.94 & 0.29
\end{tabular}

Table 7. Mean and standard deviation of reaction times for cardinally- and obliquelyoriented stimuli during $45^{\circ}$ head tilt with $0^{\circ}$ and $45^{\circ}$ scene tilt. 


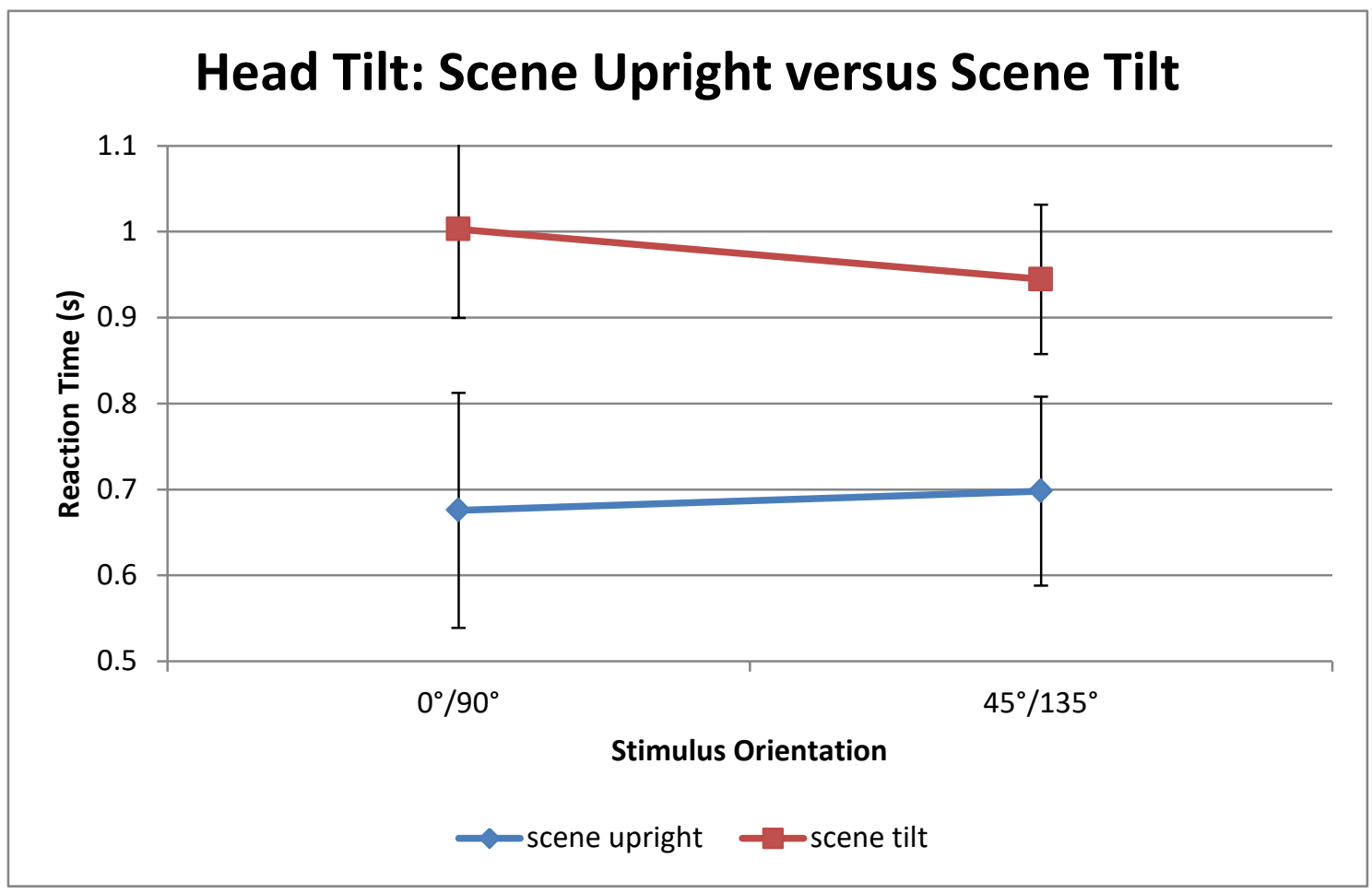

Figure 17. Reaction time data for cardinal and oblique stimulus orientations during head tilt with no scene tilt, and head tilt with $45^{\circ}$ scene tilt. Error bars represent standard error.

The final condition explored any top-down cognitive effect on the Class 2 oblique effect, as it compared head tilt with no additional thoughts to head tilt while participants were instructed to constantly think of the top of their head as up, or as defining vertical. Reaction times to cardinally- and obliquely-oriented stimuli with no additional thoughts were measured against reaction times to cardinally- and obliquely-oriented stimuli with a top-down cognition component (see Table 8). Here, because I predicted the Class 2 oblique effect to be tied to gravitational coordinates, it would be a higher-level visual effect and could in fact be influenced by top-down cognition. The results, however, show no significant interaction between cognition and orientation, $F(1,7)=0.05, p>0.05, \eta_{p}^{2}=$ 
0.01 and no significant main effect of cognition, $F(1,8)=2.175, p>0.05, \eta_{p}^{2}=0.25$ (see

Figure 18). There was a significant main effect of orientation, $F(1,7)=6.03, p<0.05, \eta_{p}^{2}$

$=0.46$ in that the reaction time for cardinals was less than that for oblique orientations.

Mean

Standard deviation

\begin{tabular}{r|lll} 
No thoughts & Cardinal stimuli & 0.60 & 0.23 \\
& Oblique stimuli & 0.67 & 0.22 \\
Top-down cognition & Cardinal stimuli & 0.65 & 0.27 \\
& Oblique stimuli & 0.72 & 0.29
\end{tabular}

Table 8. Mean and standard deviation of reaction times for cardinally- and obliquelyoriented stimuli during $45^{\circ}$ head tilt with no thought task and during instructions to think of the top of the head as up/defining vertical.

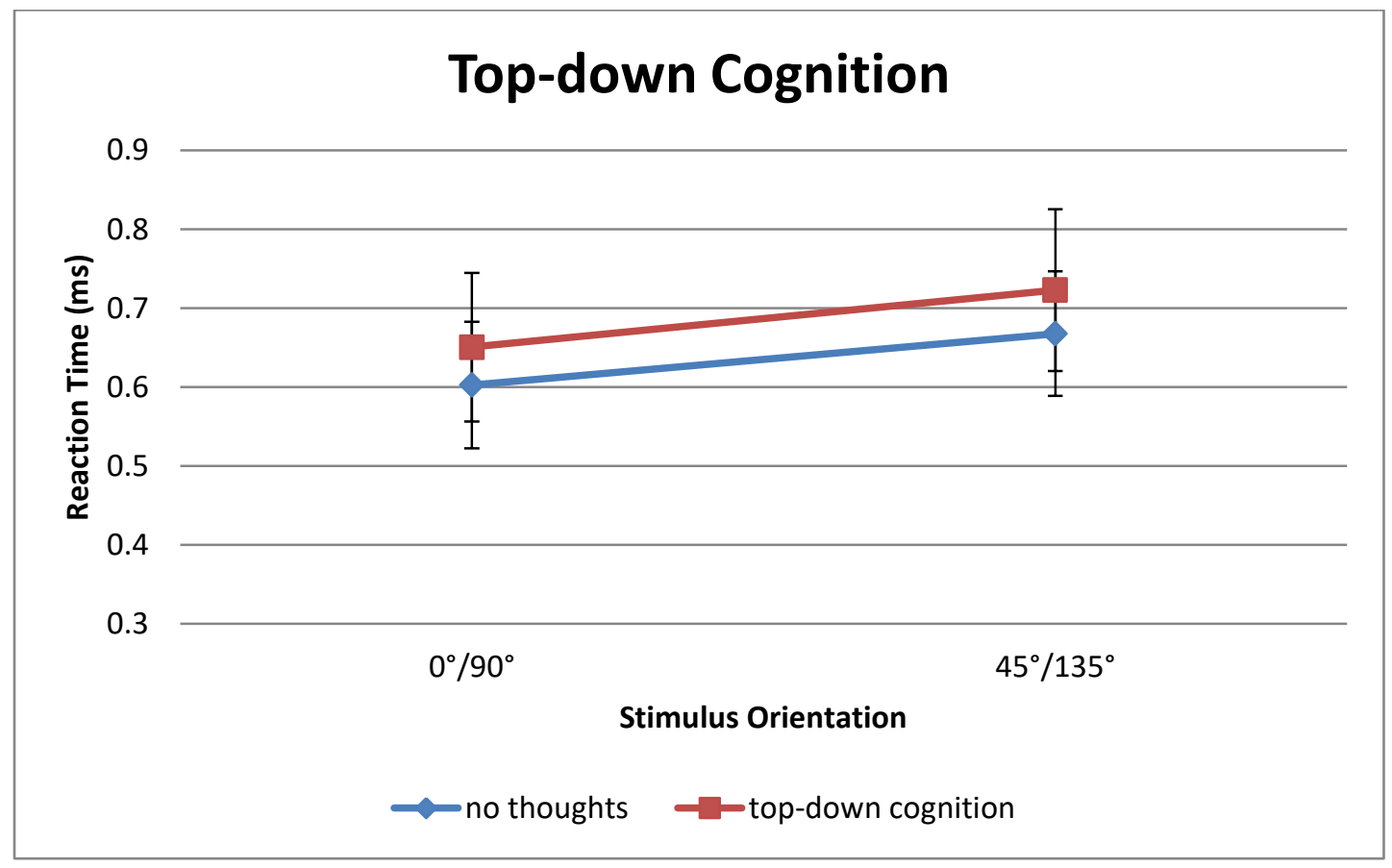

Figure 18. Reaction time data for cardinal and oblique stimulus orientations during head tilt with no additional thoughts, and head tilt while participants were instructed to constantly think of the top of their head as up. Error bars represent standard error. 
Summary. This experiment was conducted both to add evidence that the oblique effect can be divided into two classes, and to study how the visual system defines orientation coordinates for the Class 2 oblique effect. The task and stimuli used in this experiment fits the definition of Class 2, as it measures the internal representation of orientation as a frame of reference, and how we store orientations in memory (Essock \& Siqueland, 1981; Corbett \& Carrasco, 2011). The results show that the Class 2 oblique effect is defined by gravitational coordinates when retinal and gravitational coordinates are in conflict as with both heads upright and tilted, reaction times for the cardinally-oriented Gabor patches were faster than the reaction times for the obliquely-oriented Gabor patches. However, the effect is changed by patterncentric coordinates, as evidenced by the fact that scene tilt had an effect on reaction times. While participants viewed the stimuli through dove prisms with no scene tilt (the control condition), results showed the standard oblique effect of faster reaction times for cardinally- than for obliquely-oriented stimuli. With the visual scene tilted $45^{\circ}$ to the left, the effect also tilted so that participants now had faster reaction times for the oblique than the cardinal stimuli, gravitationally defined. The tilt of the oblique effect pattern could be due to a change in retinocentric coordinates, but is most likely due to the change in patterncentric coordinates because there was no change during the head tilt condition.

To explore further, the head tilt + prism conditions were analyzed. When the scene remained upright (control), but head was tilted, I expected the results show the normal oblique effect pattern gravitationally/patterncentrically defined, the reaction times would be faster are for cardinal than oblique stimuli. This condition should mirror the head tilt (no prism) condition. The results from eight participants reveal that the reaction times for 
cardinal versus oblique stimuli are approximately equal. The trend was more consistent with my hypotheses in the head tilt with scene tilt condition, where the reaction time to cardinal stimuli is slower than to oblique stimuli. In this condition, the retinal coordinates are aligned with gravitational coordinates, while patterncentric coordinates are rotated to the left. This supports the notion that the changes in the Class 2 oblique effect are due to patterncentric, or scene, changes.

The final condition tested to see if the Class 2 oblique effect pattern could be changed through top down cognition by changing phenomenological coordinates. While participants' heads were tilted, they were instructed to think of the top of the head as up, or defining vertical. The results were the same as the head tilt condition, where coordinates remained gravitationally defined and reaction times were faster for cardinally- than obliquely-oriented stimuli. Therefore, the top-down cognition did not cause any change in coordinates, which was not in line with my hypothesis (Attneave \& Olson, 1968; Reid, 1997). However, because scene tilt did affect the coordinates of the Class 2 effect, but simple head tilt did not, perhaps the Class 2 effect is more important for overall scene perception than for it to be easily malleable by top-down cognition.

The results here supporting that the human visual system defines the oblique effect pattern with respect to patterncentric coordinates is in agreement with previous articles studying the coordinates of the oblique effect using stimuli and tasks that can be classified as activating mid- to high-level processes (Luyat et al, 2005; Mend \& Qian, 2005). Many other papers using these types of stimuli conclude that the oblique effect is tied to gravitational coordinates (Attneave \& Olson, 1967; Buchanan-Smith \& Heeley, 1993; Ferrante, Gerbino \& Rock, 1995; Comerford, Javid \& Thorn, 2000). However, the 
conditions in these papers only included head upright and head tilt (sometimes prone) and so the gravitational and patterncentric coordinates were always aligned. Though it is important to note that during the head upright versus head tilt comparison, the room was dark and so the participants were not getting much, if any scene information. In this case, the effect did not change with head tilt, and so it is likely that without scene information, the effect relies on gravitational coordinates, which does agree with the above authors. The tasks used that show the oblique effect uses gravitational coordinates include orientation detection, orientation identification, orientation matching, clockwise and counterclockwise discrimination, the Goldmeier effect, and the Hermann grid illusion.

These results differ from the first experiment and with papers that used tasks and stimuli that can be classified as activating low-level visual processes, which find the oblique effect to be tied to retinal coordinates (Banks \& Solarz, 1975; Frost \& Kaminar, 1975; Corwin, Moskowitz-Cook \& Green; Chen \& Levi, 1996; Corbett \& Carrasco, 2011). This is why I argue that there are two classes of the oblique effect. If there were only one oblique effect, the same results would have occurred for both of the experiments. However, in experiment 1 the anisotropic pattern changed along with retinal coordinates, (the Class1 oblique effect), and in experiment 2 the pattern was defined by patterncentric coordinates unless scene information was unavailable, and then relied on gravitational coordinates. 
CHAPTER V

EXPERIMENT 3: THE HORIZONTAL EFFECT

\section{Experiment 3: Visual Reference frame defining the Horizontal Effect}

Hypothesis. The horizontal effect will not strictly follow retinal coordinates

during head tilt, but will also be influenced by the gravitational reference frame.

Aim. To better characterize the horizontal effect by putting retinal and

gravitational reference frames into conflict as well as test the influence of both patterncentric and phenomenological coordinates.

Rationale. Any significant diminution of the 'standard' suppression anisotropy's magnitude in relation to retinal coordinates will be taken as evidence that the anisotropy of gain control is not 'hard-wired' in early visual orientation encoding, but instead changes when reference coordinates change. While I hypothesize that this is the case, it is possible that the anisotropy may not be conserved completely; a change in retinal coordinates may shift perceived orientations away from a purely gravitational axis. If the visual system does update its coordinates that define orientation for suppression by remaining unchanged as defined by gravitational coordinates, it will indicate strong feedback connections with higher cortical areas. 


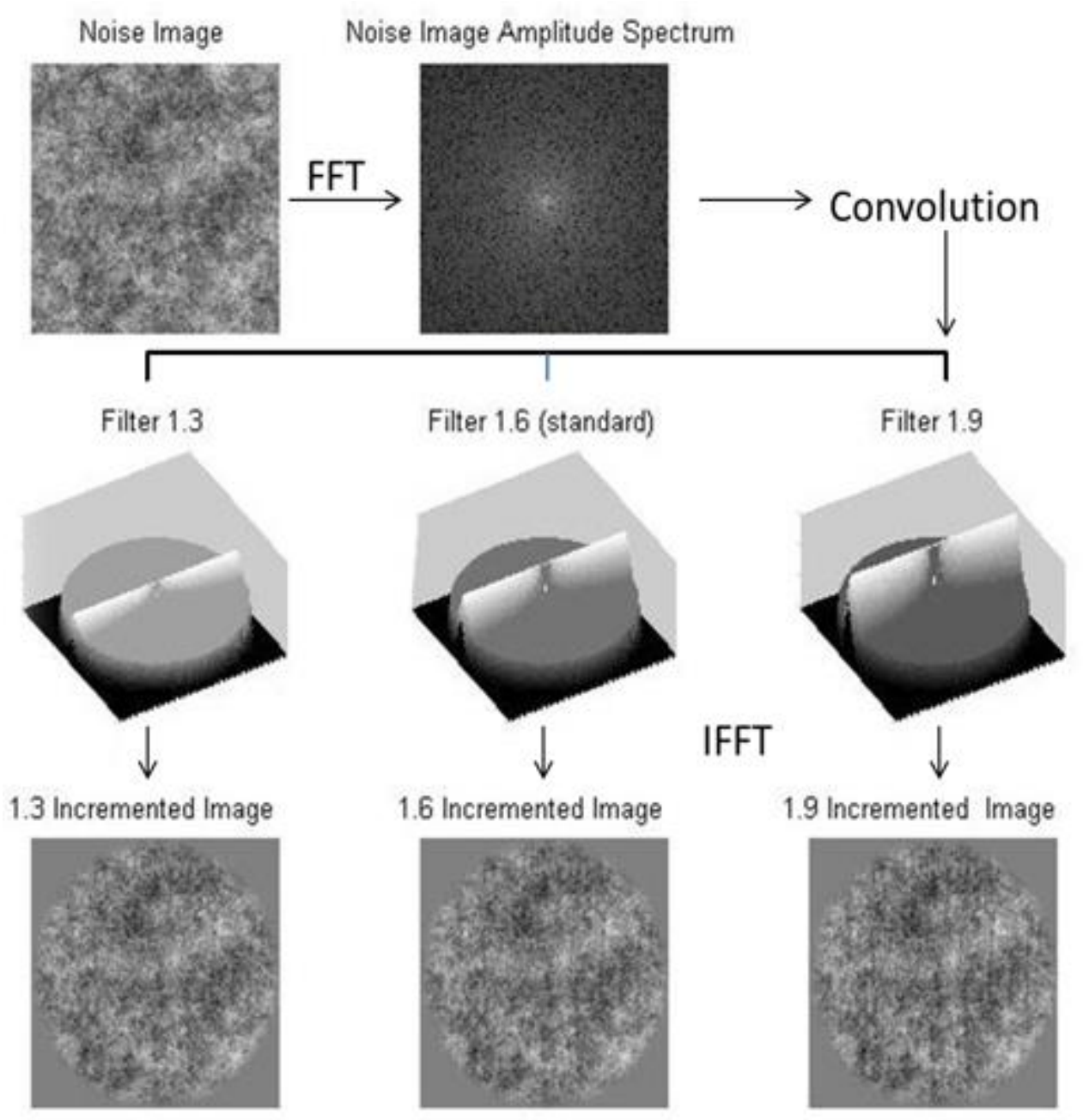

Figure 19. How the contrast matching stimuli are created. The 1.6 incremented image was the reference, at a tilt of $112.5^{\circ}$. The test stimuli are presented randomly with increments between 1.3 and 1.9 in steps of 0.5 amplitude of the triangle filter. (Hansen \& Essock, 2006; Schweinhart, Shafto \& Essock, 2015).

Procedure. The horizontal effect task was a matched contrast experiment. The stimuli were oriented broadband noise images constructed in the Fourier domain by combining random (each trial) phase spectra with a broad amplitude spectrum from frequencies 0.2 to $17 \mathrm{cpd}$ with a slope of -1 to mirror the bias found in natural scenes. The stimuli were then filtered using a triangle filter with a bandwidth of $45^{\circ}$ centered on $0^{\circ}$, $45^{\circ}, 90^{\circ}$, or $135^{\circ}$ for the test stimulus and centered on $112.5^{\circ}$ for the reference stimulus. The amplitude of the filter for the reference stimulus was set to be 1.6 times the 
magnitude of the background (see Figure 19), and the test stimulus was presented with the triangle filter amplitude randomly 1.3 to 1.9 times background magnitude (in steps of 0.5). Before display, all stimuli were then converted back to the spatial domain by inverse Fourier transform. All stimuli had 0.35 root-mean-square contrast and $80 \mathrm{~cd} / \mathrm{m}^{2}$ mean luminance. The reference and test each occupied a visual angle of $10^{\circ}$, and were separated by $1.5^{\circ}$

The reference and test stimulus were shown simultaneously on two separate monitors, with the reference stimulus always on the right. One was a Samsung SyncMaster monitor and the other a NANAO FlexScan F2-21, both with a resolution of $800 \times 600$ pixels and a refresh rate of $85 \mathrm{~Hz}$. They had matched calibrations to ensure the same luminance output, the average of which was $80 \mathrm{~cd} / \mathrm{m}^{2}$. Participants changed the amplitude of the oriented bandwidth (filter) of the stimulus on the right screen to match their perception of the strength of the orientation on the left screen. They did this by pressing 7 or 4 on the number pad in order to make a small increase or decrease in magnitude of 0.05 and press 8 or 5 to make a large increase or decrease of 0.1 . The minimum possible value was 1.3 and the maximum is 1.9 . The participants had to make a minimum of five key presses before moving on to the next trial to ensure that they were carefully evaluating the stimuli. The only differences between the comparison and test stimuli were the orientation bandwidth and the contrast scalar. This was to ensure that trial outcome is based on the contrast of the orientations rather than some other discrimination.

Analyses. Repetitions of the contrast matching measurement were averaged for analysis of the horizontal effect pattern. Differences across orientations were analyzed 
using four repeated-measures, within-factors $2 \times 2$ ANOVAs. The key was to assess how contrast matching differs between four orientations $\left(0^{\circ}, 45^{\circ}, 90^{\circ}\right.$, and $\left.135^{\circ}\right)$ with: head upright compared to $45^{\circ}$ head tilt, head upright with no prism goggles compared to head upright with prism goggles tilting the visual image $45^{\circ}$, head upright with prism goggles tilting the visual image $45^{\circ}$ compared to $45^{\circ}$ head tilt with prism goggles tilting the image $45^{\circ}$, and $45^{\circ}$ head tilt with no instruction compared to $45^{\circ}$ head tilt with the instruction to think of the top of the head as "up" or vertical. Based on pilot data with the matched contrast design, a power analysis showed that 16 participants were needed for the horizontal effect task, based on $\alpha=0.05, \beta=0.95$, and an effect size of $f=0.91$. This is double the amount of the other experiments because here I will be looking across all four orientations, and not collapsing into cardinal versus oblique orientation.

Results. As proposed, each comparison was completed using a $2 \times 4$ within-factors ANOVA. No participants were excluded from analysis. First, the head upright condition was compared to $45^{\circ}$ head tilt. Here, the influence of head tilt $\left(0^{\circ}\right.$ and $\left.45^{\circ}\right)$ and stimulus orientation $\left(0^{\circ}, 45^{\circ}, 90^{\circ}\right.$ and $\left.135^{\circ}\right)$ on the participants' perception of orientation strength was studied (see Figure 20; all of the graphs represent the data in terms of their gravitational coordinates, regardless of head tilt or scene tilt, or both. See Table 9). In this experiment, participants changed the strength of the orientation by altering the amplitude of the triangle filter. The ratio of the amplitude of the filter in the test stimulus to the amplitude of the filter of the reference stimulus (always 1.6 times the background, achieved by dividing the test amplitude by 1.6) was used to analyze the data. There was a significant interaction effect between head tilt and orientation, $F(2.26,33.82)=7.35, p<$ $0.05, \eta_{p}^{2}=0.33$, Greenhouse-Geisser corrected. Looking at Bonferroni corrected pairwise 
comparisons, $0^{\circ}$ and $135^{\circ}$ were both significantly changed as a result of the significant interaction effect. There were no significant main effects of head tilt, $F(1,15)=2.04, p>$ $0.05, \eta_{p}^{2}=0.12$, or of orientation, $F(1.92,28.74)=2.85, p>0.05, \eta_{p}^{2}=0.16$, GreenhouseGeisser corrected. With head upright, results show the standard horizontal effect pattern with the ratio for horizontal stimuli the highest and the ratio for the two obliques the lowest, with vertical stimuli falling in between. Perceptually this means that the participants need more horizontal content for the test stimulus strength of orientation to match the strength of orientation they perceive in the reference stimulus. The results show that this pattern does change with head tilt, but it is not entirely shifted to stay with retinal coordinates. If it were, gravitational $135^{\circ}$ would have the highest amplitude ratio, while $0^{\circ}$ and $90^{\circ}$ would have the lowest ratio, and $45^{\circ}$ in-between.

\begin{tabular}{c|lll}
\multicolumn{2}{c}{} & Mean & Standard deviation \\
\hline Head upright & $0^{\circ}$ & 1.05 & 0.12 \\
& $45^{\circ}$ & 0.99 & 0.081 \\
& $90^{\circ}$ & 1.01 & 0.079 \\
& $135^{\circ}$ & 0.97 & 0.097 \\
$0^{\circ}$ & 0.99 & 0.11 \\
& $45^{\circ}$ & 0.96 & 0.12 \\
$90^{\circ}$ & 0.97 & 0.10 \\
& $135^{\circ}$ & 1.02 & 0.083
\end{tabular}

Table 9. Mean and standard deviation of amplitude ratios for cardinally- and obliquely-oriented stimuli during $0^{\circ}$ and $45^{\circ}$ head tilt. 


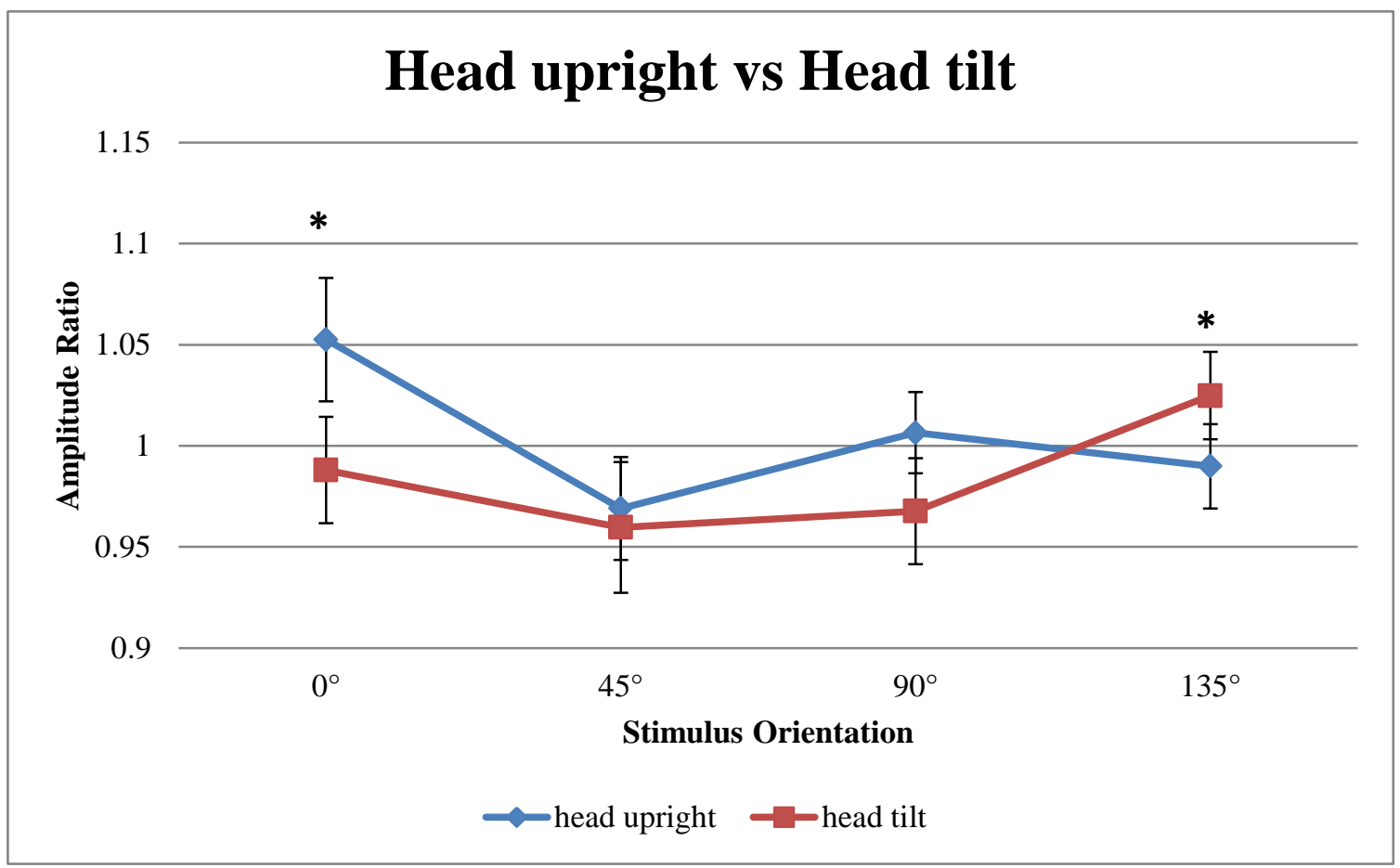

Figure 20. Ratio of the amplitude of the filter in the test stimulus to the amplitude of the filter in the reference stimulus needed for the participant to consider the strength of orientations equal. For test stimulus orientations of $0^{\circ}, 45^{\circ}, 90^{\circ}$ and $135^{\circ}$. For both head upright and head tilt to the right of $45^{\circ}$. Error bars represent standard error. Both $0^{\circ}$ and $135^{\circ}$ had significantly different amplitude ratios from their values during head upright to their values during head tilt, $p<0.05$.

Next, the scene upright condition was compared to the $45^{\circ}$ scene tilt condition (see Figure 21), specifically at the amplitude ratios during no scene tilt for $0^{\circ}, 45^{\circ}, 90^{\circ}$ and $135^{\circ}$ stimulus orientations versus the mean amplitudes during scene tilt for the same stimulus orientations (see Table 10). There was a significant interaction effect between scene tilt and orientation, $F(3,45)=6.813, p<0.01, \eta_{p}^{2}=0.31$. Looking at Bonferroni corrected pairwise comparisons, $0^{\circ}$ was significantly changed as a result of the significant interaction effect. Neither of the main effects were significant: not for orientation $F(1.92,28.86)=1.96, p>0.05, \eta_{p}^{2}=0.12$, Greenhouse-Geisser corrected, nor for scene 
tilt $F(3,45)=1.56, p>0.05, \eta_{p}^{2}=0.09$. This is unsurprising as changing head tilt did change the horizontal effect pattern, so it can be concluded that the effect is influenced by retinal coordinates. Therefore, I can already hypothesize that scene tilt will also change the horizontal effect; if not because of the patterncentric coordinate change then at least because of the retinal coordinate change. The data shows much more of a change here with scene tilt in that the pattern changes completely so that with scene upright the stimulus orientations that elicited a higher amplitude ratio, elicit a low amplitude.

\begin{tabular}{c|lcc} 
& & Mean & Standard deviation \\
\hline Scene upright & $0^{\circ}$ & 1.08 & 0.14 \\
& $45^{\circ}$ & 1.03 & 0.14 \\
& $90^{\circ}$ & 1.06 & 0.12 \\
& $135^{\circ}$ & 1.04 & 0.15 \\
& $0^{\circ}$ & 0.99 & 0.12 \\
& $45^{\circ}$ & 1.02 & 0.12 \\
$90^{\circ}$ & 1.01 & 0.12 \\
& $135^{\circ}$ & 1.07 & 0.12
\end{tabular}

Table 10. Mean and standard deviation of amplitude ratios for cardinally- and obliquely-oriented stimuli during $0^{\circ}$ and $45^{\circ}$ scene tilt. 


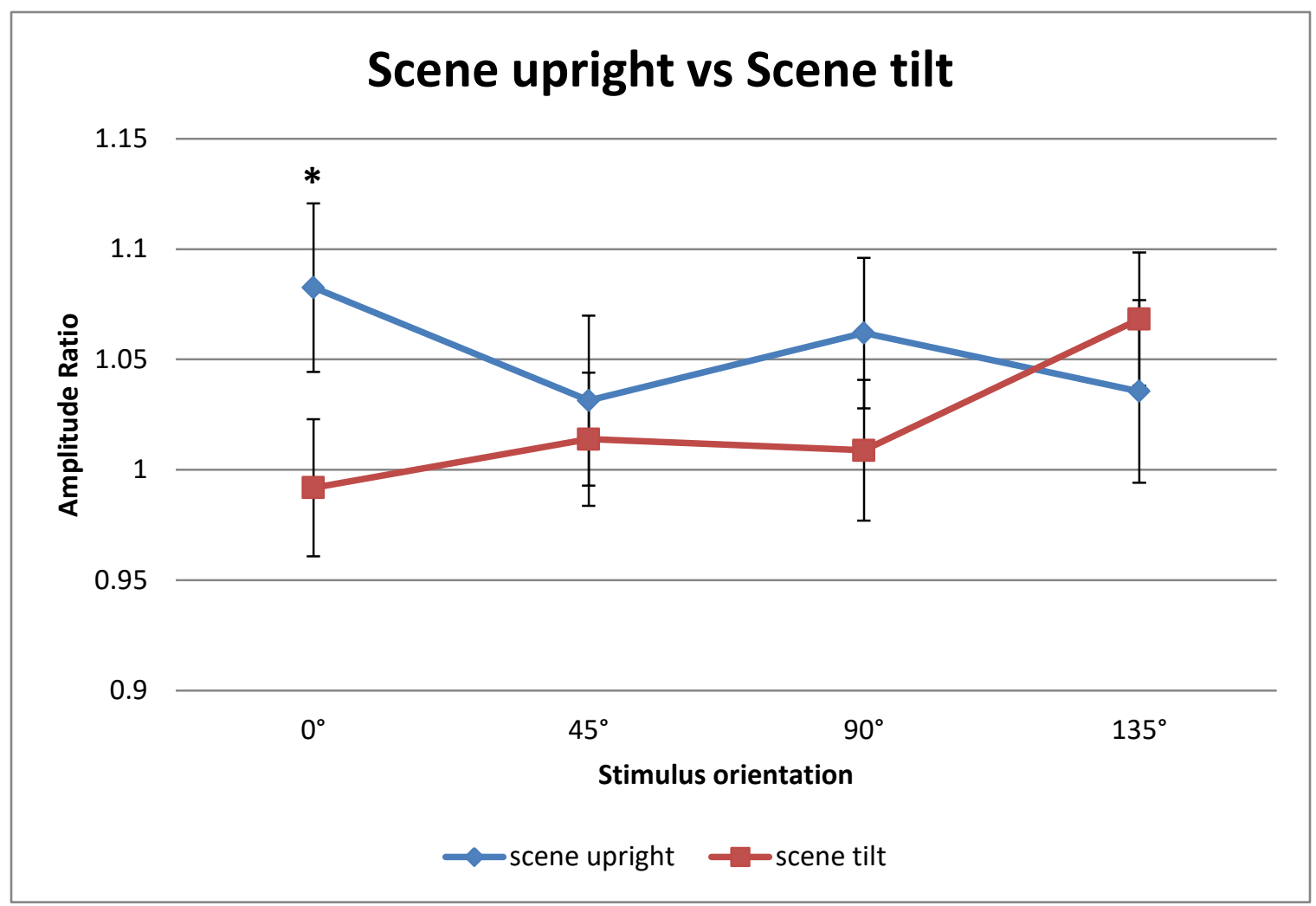

Figure 21. Ratio of the amplitude of the filter in the test stimulus to the amplitude of the filter in the reference stimulus needed for the participant to consider the strength of orientations equal. For test stimulus orientations of $0^{\circ}, 45^{\circ}, 90^{\circ}$ and $135^{\circ}$. For both scene upright and $45^{\circ}$ scene tilt. Error bars represent standard error. $0^{\circ}$ had a significantly different amplitude ratio during scene upright as compared to scene tilt, $p<0.05$.

Next, scene tilt during head tilt was compared to scene tilt during head tilt. This meant that gravitationally the coordinates remained the same, patterncentrically the coordinates were tilted $45^{\circ}$ to the left, and retinally the coordinates were the same as gravitational because the $45^{\circ}$ to the right head tilt essentially cancels out the scene tilt. Therefore, the conditions were: head tilt with no scene tilt for stimulus orientations of $0^{\circ}$, $45^{\circ}, 90^{\circ}$ and $135^{\circ}$ as compared to head tilt with scene tilt for stimulus orientations of $0^{\circ}$, $45^{\circ}, 90^{\circ}$ and $135^{\circ}$ (see Table 11 ). There was no significant interaction between scene tilt and orientation, $F(3,45)=1.31, p>0.05, \eta_{p}^{2}=0.08$, nor was there a significant main 
effect of orientation, $F(3,45)=1.30, p>0.05, \eta_{p}^{2}=0.079$ (see Figure 22). There was a significant main effect of scene tilt, $F(1,15)=5.06, p<0.05, \eta_{p}^{2}=0.25$, with amplitude ratio during head tilt and scene tilt higher than that during head tilt with no scene tilt. It is easy to see from the figure that both curves are quite flat, but curve a bit in opposing directions, which must be due to the $45^{\circ}$ difference in scene tilt.

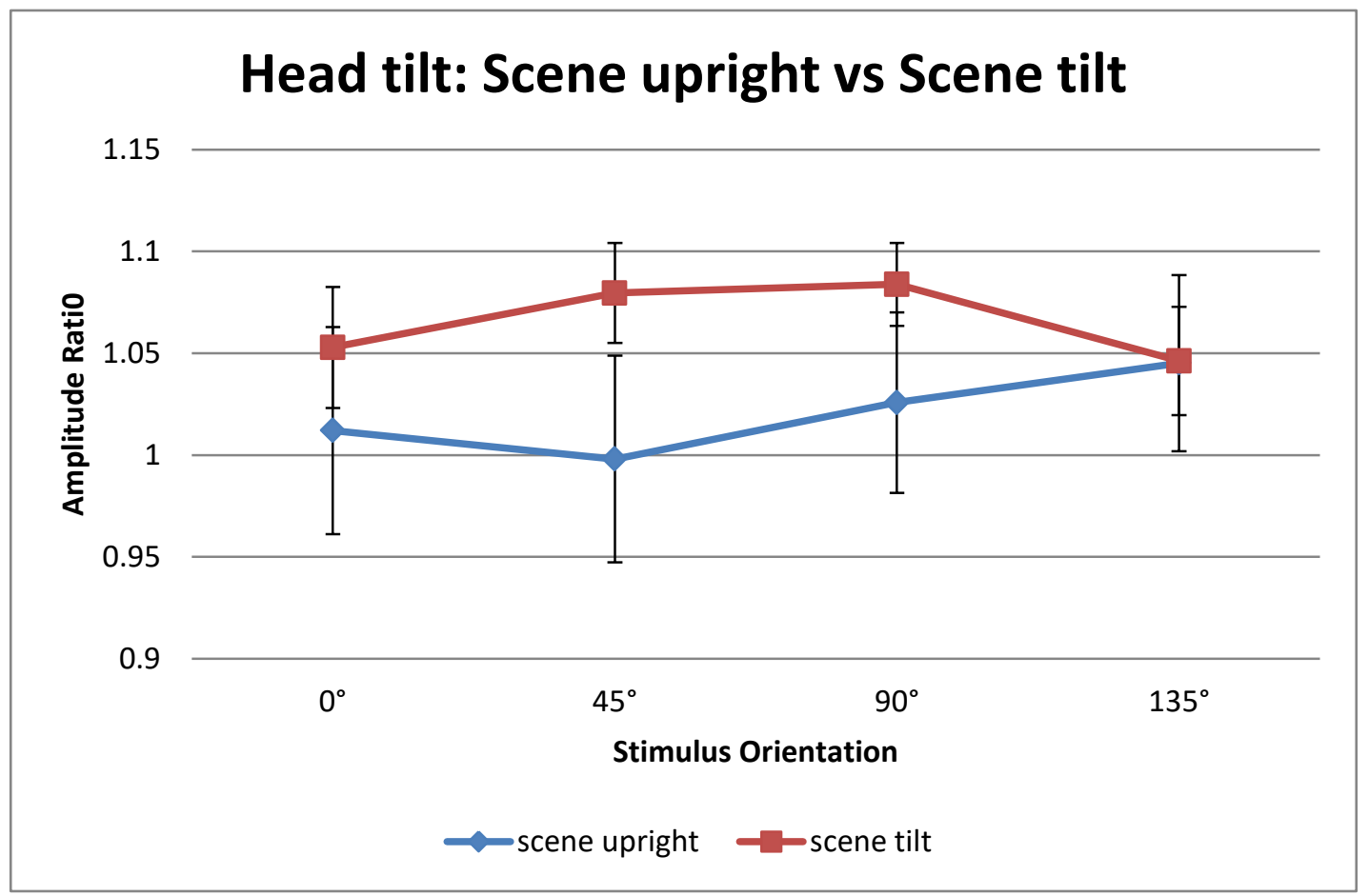

Figure 22. Ratio of the amplitude of the filter in the test stimulus to the amplitude of the filter in the reference stimulus needed for the participant to consider the strength of orientations equal. For test stimulus orientations of $0^{\circ}, 45^{\circ}, 90^{\circ}$ and $135^{\circ}$. For both head tilt with scene upright and head tilt with $45^{\circ}$ scene tilt. Error bars represent standard error. 


\begin{tabular}{l|lcc} 
Scene upright & $0^{\circ}$ & 1.08 & 0.14 \\
& $45^{\circ}$ & 1.03 & 0.14 \\
$90^{\circ}$ & 1.06 & 0.12 \\
$135^{\circ}$ & 1.04 & 0.15 \\
& $\begin{array}{l}0^{\circ} \\
45^{\circ}\end{array}$ & 0.99 & 0.12 \\
$90^{\circ}$ & 1.02 & 0.12 \\
& $135^{\circ}$ & 1.01 & 0.12 \\
& 1.07 & 0.12
\end{tabular}

Table 11. Mean and standard deviation of amplitude ratios for cardinally- and obliquely-oriented stimuli during $45^{\circ}$ head tilt for both $0^{\circ}$ and $45^{\circ}$ scene tilt.

Lastly, I wanted to know if top-down cognition had an effect and so participants were instructed to constantly think of the tops of their heads as "up"/defining vertical during $45^{\circ}$ head tilt. Therefore, the conditions were: head tilt with no additional thoughts for stimulus orientations of $0^{\circ}, 45^{\circ}, 90^{\circ}$ and $135^{\circ}$ as compared to head tilt with the topdown cognition component for stimulus orientations of $0^{\circ}, 45^{\circ}, 90^{\circ}$ and $135^{\circ}$ (see Table 12). There was no interaction between head tilt and orientation: $F(3,45)=0.698, p>$ $0.05, \eta_{p}^{2}=0.19$ (see Figure 23 ) nor a main effect of cognition $F(1,15)=0.74, p>0.05$, $\eta_{p}^{2}=0.137$. There was, however, a main effect of orientation $F(3,45)=0.04, p<0.01, \eta_{p}^{2}$ $=0.98$. Therefore, pairwise comparisons, Bonferroni corrected (see Table 13) were analyzed. The data show us that the top-down cognition did not have an effect on the results as they are still "in-between" gravitational and retinal coordinates and do not shift 
closer to retinal coordinates. What I did take a closer look at, are the differences between orientations here as there was a main effect of orientation. A significantly higher amplitude ratio was found for the $135^{\circ}$ stimulus, gravitationally defined, when compared to the $45^{\circ}$ and $90^{\circ}$ stimuli, but not the $0^{\circ}$ stimuli. Notice that when the head is tilted $45^{\circ}$ to the right, $135^{\circ}$ gravitationally is $0^{\circ}$ retinally. This tells us that with the head tilted, horizontal (according to retinal coordinates) needed a higher amplitude to be matched to the reference stimulus than vertical and $135^{\circ}$. However, it was not significantly higher than $45^{\circ}$ retinally, which is horizontal gravitationally. This definitively shows that the effect does not fully tilt from gravitational coordinates to retinal coordinates during head tilt and that horizontal defined both gravitationally and retinally are more difficult to perceive.

Mean Standard deviation

\begin{tabular}{c|lll} 
No thoughts & $0^{\circ}$ & 0.98 & 0.10 \\
& $45^{\circ}$ & 0.97 & 0.12 \\
& $90^{\circ}$ & 0.96 & 0.10 \\
& $135^{\circ}$ & 1.03 & 0.08 \\
& $0^{\circ}$ & 0.98 & 0.10 \\
& $45^{\circ}$ & 0.94 & 0.12 \\
& $90^{\circ}$ & 0.95 & 0.10 \\
& $135^{\circ}$ & 1.03 & 0.10
\end{tabular}

Table 12. Mean and standard deviation of reaction times for cardinally- and obliquelyoriented stimuli during $45^{\circ}$ head tilt with no thought task and during instructions to think of the top of the head as up/defining vertical. 


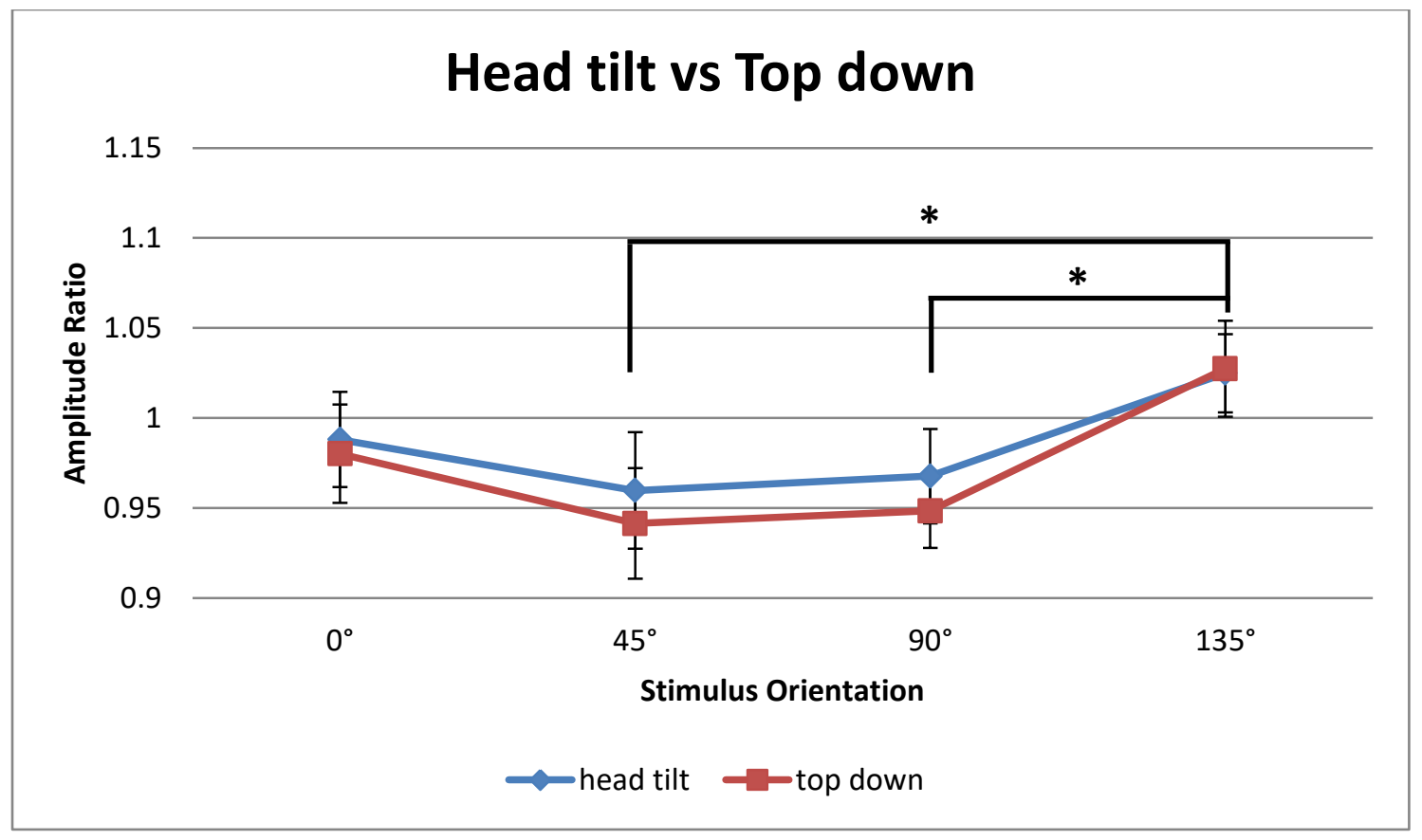

Figure 23. Ratio of the amplitude of the filter in the test stimulus to the amplitude of the filter in the reference stimulus needed for the participant to consider the strength of orientations equal. For test stimulus orientations of $0^{\circ}, 45^{\circ}, 90^{\circ}$ and $135^{\circ}$. For both no cognitive component and the cognitive top-down component. Asterisks indicate significant differences, Bonferroni corrected at $p<0.05$. Error bars represent standard error.

\begin{tabular}{|c|c|c|c|c|c|c|}
\hline \multirow[t]{3}{*}{ Orientation } & \multirow[t]{3}{*}{ Orientation } & \multirow[t]{3}{*}{$\begin{array}{l}\text { Mean } \\
\text { Difference }\end{array}$} & \multirow[t]{3}{*}{ Std. Error } & \multirow[t]{2}{*}{ Sig } & \multicolumn{2}{|c|}{$\begin{array}{l}95 \% \text { Confidence } \\
\text { Interval }\end{array}$} \\
\hline & & & & & Lower & Upper \\
\hline & & & & & Bound & Bound \\
\hline \multirow[t]{3}{*}{$0^{\circ}$} & $45^{\circ}$ & 0.026 & 0.020 & 1.000 & -0.036 & 0.088 \\
\hline & $90^{\circ}$ & 0.025 & 0.013 & 0.411 & -0.014 & 0.064 \\
\hline & 135 & -0.048 & -0.048 & 0.105 & -0.103 & 0.007 \\
\hline \multirow[t]{2}{*}{$45^{\circ}$} & $90^{\circ}$ & -0.001 & 0.017 & 1.000 & -0.051 & 0.049 \\
\hline & $135^{\circ}$ & -0.074 & 0.019 & $0.009 *$ & -0.133 & -0.016 \\
\hline $90^{\circ}$ & $135^{\circ}$ & -0.073 & 0.018 & $0.006 *$ & -0.128 & -0.019 \\
\hline
\end{tabular}

Table 13. Pairwise comparisons, Bonferroni corrected at $p<0.05$, between stimulus orientations. Asterisks denote significance. For no additional thoughts/top-down cognition comparison. 
Summary. This experiment was conducted to explore how the visual system defines orientation coordinates for the horizontal effect, as this effect pattern has never been tested with visual reference frames in conflict. The horizontal effect shows lowest thresholds for oblique and highest for horizontal orientations, with vertical orientations falling in-between (Essock, DeFord, Hansen \& Sinai, 2003; Hansen \& Essock, 2004; Essock, Haun \& Kim 2009; Kim, Haun \& Essock, 2010). It occurs with stimuli that are broadband, both in terms of in spatial frequency and orientation content, which I used in the matching task. Again, participants were instructed to change the test stimulus until the strength of perceived orientation matched that of the reference stimulus. Participants accomplished this by changing the amplitude of the triangle filter centered on that orientation, as compared to background noise. The results were analyzed using the amplitude ratio, or the filter amplitude of the reference divided by the filter amplitude of the test stimulus.

The results show the expected horizontal effect pattern during head upright, with stimuli with a triangle filter centered on $0^{\circ}$ having a higher amplitude ratio than the when the filter was centered on the obliques, with $90^{\circ}$ in-between. This means that participants needed the more horizontal content to match their perception of the strength of orientation in the reference stimulus as compared to the other orientations. However, during $45^{\circ}$ head tilt, this pattern flattens out with a slight bowing, so that it does not stay wholly with gravitational coordinates nor move completely with retinal coordinates. This implies that the effect is influenced by input from the vestibular system.

While participants viewed the stimuli through dove prisms with no scene tilt (the control), subjects exhibited the standard horizontal effect pattern with a higher amplitude 
ratio for horizontal stimuli. With the visual scene tilted $45^{\circ}$ to the left, the effect also tilted so that participants now had a higher amplitude ratio for $135^{\circ}$, gravitationally defined, or $0^{\circ}$ patterncentrically/retinally defined. The tilt of the oblique effect pattern could be wholly due to a change in retinocentric coordinates. However, with head tilt, the effect flattened and did not shift completely with retinal coordinates. It is likely that there is some shift in this condition due to the change in retinal coordinates but also due to the change in patterncentric coordinates. A combination, or intermediate influence, of the two coordinate systems may be influencing the effect to shift completely with scene tilt.

To explore further, the head tilt + prism conditions were analyzed. When the scene remained upright (control), but head was tilted, there is again a flattening of the horizontal effect pattern with slight bowing, as in the condition of head tilt with no prisms. When the head remained tilted and the scene was also tilted through the prisms, the pattern remained flattened. Here, the bowing of the pattern during head tilt with scene tilt curves the opposite way of head tilt with scene upright. During this condition, retinal and gravitational coordinates are aligned, and in disagreement with patterncentric coordinates. Putting these results together with the other comparison conditions, it looks like the horizontal effect pattern to flatten again due to a competition of retinal and patterncentric coordinates.

The final comparison tested whether the horizontal effect pattern could be changed through top down cognition by changing phenomenological coordinates. While participants' heads were tilted, they were instructed to think of the top of the head as up, or defining vertical. The results were the same as the head tilt condition, where coordinates remained in-between retinal and gravitational coordinates; therefore, the top- 
down cognition did not cause any change in coordinates. If there were cognitive effects, the pattern would have shifted closer to retinal coordinates because the phenomenological coordinates were aligned with retinal coordinates. Because there was a main effect of orientation, a closer look was taken at how the pattern is different during head tilt from the normal horizontal effect pattern. Post hoc testing, Bonferonni corrected, showed that the amplitude ratio for the stimulus at $135^{\circ}$ was significantly higher than $45^{\circ}$ and $90^{\circ}$. This shows the slight transfer of the horizontal effect pattern toward retinal coordinates as $135^{\circ}$ gravitationally is $0^{\circ}$ retinally during the $45^{\circ}$ head tilt to the right. However, it is not a complete transfer as the amplitude ratio of the $135^{\circ}$ gravitational stimulus ( $0^{\circ}$ retinal) is not significantly higher than that of the $0^{\circ}$ gravitational stimulus ( $45^{\circ}$ retinal).

The results here support that the human visual system defines the horizontal effect pattern neither by retinal nor by gravitational coordinates. This could result from weak or lack of influence from the vestibular system or, alternatively, strong suppression of horizontal orientation in both coordinates systems, which seems less likely. The horizontal effect pattern is also changed by patterncentric coordinates since tilting these coordinates tilts the effect as well. There is a complete tilt of the effect toward patterncentric coordinates when they are in alignment with retinal coordinates, but incomplete tilt when they are in disagreement with retinal coordinates. The importance of patterncentric coordinates is in agreement with the theory that the horizontal effect serves to "whiten" a natural scene. Because the natural scenes contain most orientation content (Fourier power) at horizontal, second most at vertical, and least at oblique orientations, the horizontal effect normalizes a natural scene by suppressing horizontal content the most and oblique input the least, so that typical natural scene content is suppressed and 
less-prevalent content is made relatively more salient (Hansen \& Essock, 2004; Haun, Hansen, \& Essock, 2006; Essock et al, 2009; Haun \& Essock, 2010; Kim, Haun, \& Essock, 2010). 


\section{CHAPTER VI \\ GENERAL DISCUSSION}

\section{Experiment 1: Visual Reference frame defining the Class 1 Oblique Effect}

In this experiment I set out to replicate the findings of earlier studies to investigate which reference frame the visual system uses to define the Class 1 oblique effect. The oblique effect describes the phenomenon that humans can more easily perceive cardinally-oriented than obliquely-oriented stimuli, (Appelle, 1972). In the literature, "the oblique effect" is used as an umbrella term, and there is currently a discrepancy as to whether the effect follows the retinal or gravitational reference frame. I argue that these results are dependent upon the context in which stimuli are presented. The Class 1 oblique effect specifically represents low-level visual system properties which occur post-retinally (Campbell, Kulikowski \& Levinson, 1966; Frost \& Kaminar, 1975). Therefore I looked only at previously conducted visual reference frame studies that employed tasks that measure low-level vision, including contrast sensitivity, parallelism and perpendicularity discrimination, and VEP amplitudes (Banks \& Solarz, 1975; Frost \& Kaminar, 1975; Corwin, Moskowitz-Cook \& Green; Chen \& Levi, 1996; Corbett \& Carrasco, 2011). All were in agreement that the oblique effect is defined by the retinal reference frame. To ensure the measurement of low-level visual properties in this experiment, I employed a contrast threshold task. Participants completed this task in several different conditions where visual reference frames were put into conflict. The reference frames considered were retinal, gravitational, and patterncentric, as well as 
phenomenological coordinates to test for the effects (if any) of top-down cognition. For all of these conditions, I hypothesized that the Class 1 oblique effect would follow retinal coordinates.

In the first comparison, where participants completed the contrast threshold task both with their heads upright and tilted, the Class 1 oblique effect pattern did change with retinal coordinates. Note that in this particular comparison, no patterncentric (scene) information was available to participants. There was also a main effect of head tilt, which was unexpected. Looking at the data (Figure 11), it seems as though cardinal stimulus thresholds were the only ones to change, while the oblique thresholds remained the same for both head upright and head tilt conditions. This could suggest that cardinal thresholds are more easily malleable in early vision, while oblique thresholds tend to remain set. However, the results are more easily explained considering the difficulty between the two conditions. Most likely, head tilt itself caused the thresholds of all stimulus orientations to increase because people rarely sit with their heads at a $45^{\circ}$ angle, otherwise, thresholds for oblique stimuli during head tilt would be lower than oblique thresholds during head upright.

The next analysis compared the scene upright and scene tilt conditions. Again, the effect pattern changed to align with retinal coordinates. In this comparison, retinal coordinates are aligned with the available patterncentric coordinates, but given the results from head upright versus head tilt, it is most likely that the change in thresholds during scene tilt is due to the change in retinal coordinates and not that of patterncentric 
coordinates. This is also confirmed by the next comparison between head tilt with scene upright and head tilt with scene tilt. During the head tilt/scene upright condition, the oblique effect pattern flipped to stay with retinal coordinates, and then when scene tilt was introduced (head tilt/scene tilt), it flipped again to the change in retinal coordinates. If the Class 1 oblique effect was defined by patterncentric coordinates, it would have remained upright during head tilt with scene upright.

Lastly, head tilt with no additional instructions was compared to head tilt with instructions for participants to think of the top of the head as up/defining vertical. This task was used to test if the Class 1 oblique effect pattern could be changed through top down cognition. The results show the same pattern for each condition - that the pattern is aligned with retinal coordinates. However, because retinal coordinates were aligned with phenomenological coordinates, it cannot be determined whether the change in phenomenological conditions had any effect. A condition with the head tilted where participants were instructed to think of gravitational "up" as up would be useful here. It is worth nothing that while not constantly reminding ourselves of what "up" is, we do still tend to consider gravitational "up" as up during head tilt (Wade, 1970; 1992; Luyat \& Gentaz, 2002; Tarnutzer, Bockisch \& Straumann 2009), such as could be the case with head tilt and no instructions. In either case, because I am postulating that the Class 1 oblique effect is caused by low-level visual system properties, it is most likely unaffected by changes in phenomenological coordinates.

These results of this experiment confirm the hypothesis that the visual system uses retinal coordinates to define the Class 1 oblique effect. They also successfully replicate the findings of authors who use tasks that measure low-level vision to study the 
oblique effect (Banks \& Solarz, 1975; Frost \& Kaminar, 1975; Corwin, Moskowitz-Cook \& Green; Chen \& Levi, 1996; Corbett \& Carrasco, 2011).

\section{Experiment 2: Visual Reference frame defining the Class 2 Oblique Effect}

In this experiment, the intent was to confirm and extend the findings of earlier studies conducted on what reference frame the visual system uses to define the Class 2 oblique effect. The Class 2 oblique effect specifically represents higher-level visual properties such as categorization and memory. It is thought to be due to trouble distinguishing between different oblique orientations, while horizontal and vertical orientations remain easily discernible, which leads to better classification of cardinals (Essock, 1980; Luyat \& Gentaz, 2002). Therefore in this section I looked only at visual reference frame studies that employed higher-level tasks including orientation detection, identification and matching, clockwise and counterclockwise discrimination, the Goldmeier effect, and the Hermann grid illusion, (Attneave \& Olson, 1967; BuchananSmith \& Heeley, 1993; Ferrante, Gerbino \& Rock, 1995; Comerford, Javid \& Thorn, 2000). These authors concluded that the oblique effect is defined by gravitational coordinates. The reaction time task used to measure the Class 2 oblique effect was based on the tasks used in Essock (1980) and Haun, Hansen, Kim, and Essock (2005). In the task here, oriented Gabor patches appeared on screen and participants were instructed to classify them correctly and as fast as possible by their orientation. The same reference frames were considered as in experiment 1: retinal, gravitational, patterncentric and phenomenological coordinates. 
I hypothesized that the results would show that the Class 2 oblique effect is tied to gravitational coordinates, in agreement with the above literature. However, I was uncertain whether this gravitational preference would hold true during the scene tilt conditions. This is because Class 2 oblique effect tasks have also been shown to be influenced by the patterncentric reference frame (Prather, 1997; Luyat et al, 2005; Meng \& Qian, 2005). Therefore, I predicted that during the scene tilt condition, the patterncentric coordinates could override the gravitational coordinates and the effect pattern would shift with scene tilt. If this were true, the effect pattern would shift with scene tilt during the combined scene tilt and head tilt condition. Attneave and Reid (1968) and Prather (1997) also found the Class 2 effect can be influenced by phenomenological coordinates, or at least a combination of visual and phenomenological cues. Therefore, I expected that during the condition where subjects were instructed to think of the top of their head as "up", the effect would shift toward head tilt.

The findings did agree with the majority of my predictions. When participants' heads were both upright and tilted (with no scene information available), the Class 2 oblique effect was defined by the gravitational reference frame. Inducing scene tilt through the use of dove prisms did cause the Class 2 effect pattern to shift to the new patterncentric coordinates. However, there was no interaction effect in the comparison of head tilt with scene upright to head tilt with scene tilt. This could be due to the difficulty of the head tilt/scene tilt condition where participants had to ignore both the scene tilt and head tilt while continuing to categorize the stimuli. It is possible that one could elicit stronger effects with a slightly different experimental design. In order to replicate the Haun et al (2005) study, the stimuli were $0.5^{\circ}$, though not oriented line stimuli. The 
stimuli in this experiment were oriented Gabor patches so that the stimulus type would match that of experiment 1 , in order to help show that task difference matters between the Class 1 and 2 oblique effects. These changes meant that stimulus orientation was more difficult to perceive, which would affect the results the most during the more difficult tasks, such as head tilt with scene tilt.

There was no change in coordinates due to top-down cognition. This is only partially conflicts with Prather's (1997) work. His cues included a white arrow as well as optic flow moving in the direction of "up", so that there was a visual cue present for participants. No visual orienting cues were used in the phenomenological condition. This could mean that instructing participants to think of the tops of their heads as up is not a strong enough cue, or that in the Prather (1997) study, the change was due simply to patterncentric coordinates and not the combination of patterncentric and phenomenological coordinates. In addition, Attneave and Reid (1968) found a trend towards a change in coordinate systems with a change in phenomenological coordinates, but it was not a significant change.

These results do confirm that the visual system uses gravitational coordinates to define the Class 2 oblique effect, but only in part. During head tilt with no scene information, the effect pattern remains tied to gravitational coordinates. However, in cases where scene information was available, the results show that the visual system uses patterncentric coordinates to define the effect. It is important to know that the authors who used tasks that measure higher-level vision to study the oblique effect and conclude that it is defined by gravitational coordinates only employ head tilt, so that gravitational and patterncentric coordinates could not be parsed apart (Attneave \& Olson, 1967; 
Buchanan-Smith \& Heeley, 1993; Ferrante, Gerbino \& Rock, 1995; Comerford, Javid \& Thorn, 2000). The results here replicate authors who have specifically looked at patterncentric coordinates and found that the oblique effect pattern changes (Prather, 1997; Luyat et al, 2005; Meng \& Qian, 2005).

\section{Experiment 3: Visual Reference frame defining the Horizontal Effect}

While the horizontal effect has been thoroughly studied, it has not yet with respect to different visual coordinate systems. The horizontal effect itself occurs during low-level visual tasks, but when broadband instead of narrowband stimuli are used. Instead of the oblique effect pattern, where cardinal stimuli have lower contrast thresholds than oblique stimuli, oblique stimuli now have the lowest thresholds and horizontal stimuli the highest, with vertical stimuli threshold values falling in-between (Essock, DeFord, Hansen \& Sinai, 2003). The theory behind this change is that with more information in the stimulus, the process of gain control is activated. Gain control, like the moniker suggests, operates to suppress the most common information in the environment so that our perception is not overwhelmed by this redundant information so that information content in the environment is perceived more equally, i.e. sometimes referred to as "whitening" the stimulus. When measuring the amount of orientation in an everyday natural environment, the horizontal effect seems particularly useful. In both man-made and more "natural" (no man-made structures) scenes, there is a greater amount of orientation content at horizontal, least at obliques, and vertical is in-between. Therefore, the horizontal effect would serve to whiten our everyday environments so that we perceive orientations more evenly and ignore this bias. 
Because the horizontal effect seems so important for scene perception, I hypothesized that it is likely defined by patterncentric coordinates. And, if it is important for the whitening of a scene, it shouldn't be pliable with respect to phenomenological coordinates, and therefore, I did not expect to see any change during that condition. The task used to measure the horizontal effect was designed after both Hansen and Essock (2006) and Schweinhart et al. (2015). It required participants to match their perceived strength of orientation in the test stimulus to their perceived strength of orientation in the reference stimulus. The orientation of the reference was always $112.5^{\circ}$ and the orientation of the test stimulus varied between $0^{\circ}, 45^{\circ}, 90^{\circ}$, and $135^{\circ}$. In this way, it could be determined if participants needed more or less orientation strength for the four different stimulus orientations to match their perception of the strength of orientation of the reference stimulus.

The results remain mixed as to what frame of reference the visual system uses to define the horizontal effect pattern. During the head upright condition, results showed the normal horizontal effect pattern where participants needed the most orientation content, relatively, in the horizontal stimulus to match the reference stimulus, the least content in the two oblique stimuli, while the vertical stimulus fell in-between. However, the head tilt condition showed a "flattening" of this effect pattern in that it did not shift completely with retinal coordinates, nor did it remain tied to gravitational coordinates (in this condition, patterncentric coordinates were unavailable). The horizontal effect pattern did shift completely when the visual scene was tilted so that patterncentric and retinal coordinates were in alignment. When the scene remained upright and the head was tilted, the effect failed to remain with the patterncentric/gravitational coordinates, nor was it 
pulled completely toward retinal coordinates. That is, it looked flattened as during the head tilt condition with no scene information. During the head tilt and scene tilt condition, when retinal coordinates were upright but the visual scene was tilted, the effect neither remained with retinal coordinates nor shifted completely to patterncentric coordinates. Therefore, there is this kind of tug-of-war for the effect pattern between retinal and patterncentric frames of reference. Lastly, head tilt with no instructions was compared to head tilt with instructions to think of the top of the head as up (shifting phenomenological coordinates). As predicted, the change in phenomenological coordinates had no effect on the effect pattern; both results showed the same flattened pattern seen during head tilt. If the change in phenomenological coordinates were to have an effect, the results would have moved toward those coordinates. Analyzing the main effect of orientation during this comparison allowed a closer look at the flattened effect pattern. The amplitude ratio for the $135^{\circ}$ stimulus was significantly higher than that of the $45^{\circ}$ and $90^{\circ}$ stimulus, but not the $0^{\circ}$ stimulus, in gravitational coordinates. Consider that in retinal coordinates, $135^{\circ}$ is $0^{\circ}$. One can see the incomplete pull of the effect pattern toward retinal coordinates during head tilt, where horizontal in both retinal and gravitational reference frames is more difficult to perceive.

As a whole, these results provide some clue as to where along the visual pathway the horizontal effect occurs. Even with no scene information available, the shift toward retinal coordinates is not complete during head tilt, meaning that there are occasions where gravitational coordinates play a role. Most likely, orientation signals that are both changed and unchanged by head tilt, i.e. those that are privy to vestibular information and those that aren't, are both involved in the production of the horizontal effect. And, 
because patterncentric coordinates play such an important role, whole scene information must be available where this effect occurs. Again, phenomenological coordinates do not show an effect.

\section{General Conclusions}

The purpose of this dissertation was to determine the reference frame(s) that the human visual system uses to define the coordinate systems of three different orientation anisotropies. I hoped that through doing this, the results would show a clear separation between the Class 1 and 2 oblique effects, as the majority of the time in current literature they are referred to simply as the oblique effect. The third orientation effect pattern studied was the horizontal effect, as tests involving reference frame conflict have never been applied to this effect before. To investigate the different reference frames, I varied

retinocentric, patterncentric, and gravitational coordinates through combinations of head tilt and scene tilt. In addition, the degree to which each of these anisotropies can be influenced by top down effects, or phenomenological coordinates, was measured.

The first two experiments show that the Class 1 oblique effect is defined by retinal coordinates and the Class 2 oblique effect is defined by patterncentric coordinates, though without patterncentric information available, the Class 2 effect pattern remained tied to gravitational coordinates. Most importantly, the results provide added evidence that there are two classes of the visual oblique effect and, henceforth, it should not be considered a singular construct. The results show that the Class 1 effect is low-level and likely occurs in V1 or V2, where a greater number of cells are tuned to cardinal orientations than oblique orientations, and that the cells tuned to cardinals have sharper 
tuning curves relative to those tuned to obliques (Mansfield, 1974; Rose \& Blakemore, 1974; Mansfield \& Foster, 1978; Furmanski \& Engle, 2000; Li, Peterson \& Freeman, 2003). The results further affirm that the Class 2 effect is processed at a higher-level in the visual stream and influenced by multisensory integration, making use of scene information as well as vestibular information during head tilt. It has been suggested that the Class 2 oblique effect occur at a processing stage where information is represented categorically based on the cardinal coordinate system, but is not modality specific, (Baud-Bovy \& Gentaz, 2012; Smyrnis et al, 2014). Others have looked at the parietal cortex as a possible location as it is known to process spatial information (Izyurov \& Slavutskaya, 2015). The inferior parietal cortex receives vestibular afferents and is a multisensory area that includes processing of spatial orientation (Brandt, Dieterich \& Daneck, 1994). There also exists the PIVC, which receives both vestibular and visual information and is thought to be responsible for the perception of the head in space. Further work is needed to ascertain whether the class 2 effect might occur in the parietal lobe, a more multimodal area, or in the occipital lobe where it integrates feedback from vestibular areas.

The third experiment showed that the horizontal effect is influenced by several reference frames. When no scene information was available and retinal and gravitational reference frames were in conflict, there was an incomplete shift of the horizontal effect pattern toward retinal coordinates. When patterncentric and gravitational frames were in alignment and the retinal frame in competition, the effect was pulled toward retinal coordinates but did not transfer completely. When patterncentric and retinal frames were in alignment and the gravitational frame in competition, the effect made complete a 
transfer to the retinal/patterncentric coordinates. When retinal and gravitational frames were in alignment and the patterncentric frame in competition, there was incomplete transfer toward the scene tilt. Therefore, patterncentric and retinal reference frames both exert influence on the horizontal effect, while the gravitational reference frame did so in lieu of scene information.

The horizontal effect is thought to be important for natural scene perception as it occurs when viewing broadband stimuli, i.e. the kind of stimuli we are surrounded by daily. The theory suggests that when viewing narrowband stimuli or stimuli with low signal to noise (SNR), it is useful to concentrate on dominant environmental statistics. However, when viewing broadband stimuli or stimuli with high SNR, resources are diverted to infrequent environmental statistics. Maloney and Clifford (2015) found that changing from a low to high SNR changes the greatest BOLD signal from cardinal to oblique orientations in V1, V2 and V3. In addition, the results show that the horizontal effect must be updated by vestibular input as it does not completely follow retinal coordinates during head tilt but remains partially tied to gravitational coordinates. In V2 and $\mathrm{V} 3 / \mathrm{V} 3 \mathrm{~A}$, there exist about $40 \%$ of cells whose preferred orientations were invariant with respect to gravity, i.e. compensatory, (Denney \& Adorjani, 1972; Horn, Stechler \& Hill, 1972; Sauvan \& Peterhans, 1999). It seems likely then that the horizontal effect occurs in one of these areas where the incomplete shift of the effect pattern to retinal coordinates is due to conflicting input from both compensatory and non-compensatory units.

These studies show us that orientation anisotropies are more complex than previously thought. When the oblique effect is studied, researchers must specify which 
class applies to their work, as the two classes have different properties and most likely occur in different cortical areas. Future studies should focus on narrowing down the location of the Class 2 oblique effect and horizontal effect, where neuroimaging methodology is probably most appropriate. When studying the Class 2 oblique effect, focus should be on the parietal cortex, specifically the inferior part of the cortex and the PIVC, though other multisensory areas could also be considered. From here, researchers should also look at later effects in the occipital lobe, as Mikhailova et al (2015) found a delayed response in the occipital cortex after original signal differences in the parietal cortex (possible feedback). Tasks that elicit the Class 2 oblique effect should be used, such as orientation categorization and memory tasks. Further testing with a stronger topdown cue may still show an effect of phenomenological coordinates on the Class 2 oblique effect, especially if it is combined with visual cues that serve to label the coordinates (Prather, 1997). When studies employ broadband stimuli, researchers should be aware of the horizontal effect. The studies here show that the location of this effect seems to occur quite early along the visual pathway. When studying the horizontal effect using imaging, focus should be on the occipital cortex, specifically V1, V2 and V3. Maloney and Clifford (2015) saw a switch of greatest BOLD signal in V1 from cardinals to obliques when image contrast increased, with the same, but reduced effect in V2 and V3. In addition, neurons that are compensatory and non-compensatory for gravity have been found to co-exist in V2 and V3/V3A. 


\section{Appendix 1}

\begin{tabular}{|l|r|r|r|}
\hline & Experiments & & \\
\hline Conditions & Oblique Class 1 & Oblique Class 2 & Horizontal Effect \\
\hline no head tilt vs. head tilt & 8 & 8 & 16 \\
\hline $\begin{array}{l}\text { no scene tilt (with } \\
\text { prisms) vs. } \\
\text { scene tilt with prisms }\end{array}$ & 8 & 8 & 16 \\
\hline $\begin{array}{l}\text { head tilt + no scene tilt } \\
\text { vs. } \\
\text { head tilt + scene tilt }\end{array}$ & 8 & & 16 \\
\hline $\begin{array}{l}\text { head tilt with } \\
\text { phenomenological } \\
\text { instructions }\end{array}$ & & 8 & \\
vs. head tilt & & & \\
\hline
\end{tabular}

Sample size needed for each of the conditions in each of the experiments proposed. For the Class 1 oblique effect a power analysis showed that 8 participants were needed based on $\alpha=0.05, \beta=0.95$, and an effect size of $f=1.4$. For the Class 2 oblique effect, a power analysis showed that 8 participants were needed with $\alpha=0.05, \beta=0.95$, and an effect size of $f=0.91$. For the horizontal effect task, a power analysis showed that 16 participants were needed based on $\alpha=0.05, \beta=0.95$, and an effect size of $f=0.91$. 


\section{REFERENCES}

Appelle, S. (1972). Perception and discrimination as a function of stimulus orientation: The "oblique effect" in man and animals. Psychological Bulletin, 78(4), 266-278.

Attneave, F., \& Olson, R. K. (1967). Discriminability of stimuli varying in physical and retinal orientation. Journal of Experimental Psychology, 74, 149-157.

Baddeley, R. J., \& Hancock, P. J. B. (1991). A statistical analysis of natural images matches psychophysically derived orientation tuning curves. Proceedings of the Royal Society of London B, 246, 219-223.

Banks, M. S., \& Stolarz, S. J. (1975). The effect of head tilt on meridional differences in acuity: Implications for orientation constancy. Perception \& Psychophysics, 17(1), $17-22$

Barra, J., Marquer, A., Joassin, R., Reymond, C., Metge, L., Chauvineau, V., \& Perrenou, D. (2010). Humans use internal models to construct and update a sense of verticality. Brain, 133, 3552-3563.

Baud-Bovy, G., \& Gentaz, E. (2012). The perception and representation of orientations: A study in the haptic modality. Acta Psychologica, 141, 24-30.

Bear, M. F., Connors, B. W., \& Paradiso, M. A. (2007). Neuroscience: Exploring the brain (3rd ed.). Philadelphia, PA: Lippincott Williams \& Wilkins.

Berkes, P., Orbán, G., Lengyel, M., \& Fiser, J. (2011). Spontaneous cortical activity reveals hallmarks of an optimal internal model of the environment. Science, 331, 83-87. 
Berman, P. W. (1976). Young children's use of the frame of reference in construction of the horizontal, vertical, and oblique. Child Development, 47, 259-263.

Bienenstock, E. L., Cooper, L. N., \& Munro, P. W. (1982). Theory for the development of neuron selectivity: Orientation specificity and binocular interaction in visual cortex. The Journal of Neuroscience, 2(1), 32-48.

Blakemore, C., \& Cooper, G. F. (1970). Development of the brain depends upon the visual environment. Nature, 228, 447-448.

Blanke, O., Perrig, S., Thut, G., Landis, T., \& Seeck, M. (2000). Simple and complex vestibular responses induced by electrical cortical stimulation of the parietal cortex in humans. Journal of Neurology, Neurosurgery \& Psychiatry, 69, 553-556.

Borra, T., Hooge, I., \& Verstraten, F. (2010). A dichoptic study of the oblique effect. Perception, 39, 909-917.

Brandt, T., Dieterich, M., \& Danek, A. (1994). Vestibular cortex lesions affect the perception of verticality. Annals of Neurology, 35(4), 404-412.

Buchanan-Smith, H. M., \& Heeley, D. W. (1993). Anisotropic axes in orientation perception are not retinotopically mapped. Perception, 22, 1389-1402.

Campbell, F. W., Kulikowski, J. J., \& Levinson, J. (1966). The effect of orientation on the visual resolution of gratings. Journal of Physiology, 187, 427-436.

Carandini, Matteo. "Receptive Fields and Suppressive Fields in the Early Visual System." In The Cognitive Neurosciences. By Michael S. Gazzaniga and Emilio Bizzi. Cambridge, MA: MIT, 2004. Print.

Carandini, M. (2006). What simple and complex cells compute. The Journal of Physiology, 577, 463-466. 
Carandini, M., \& Heeger, D. J. (2012). Normalization as a canonical neural computation. Nature Reviews: Neuroscience, 13, 51-62.

Chapman, B., \& Bonhoeffer, T. (1998). Overrepresentation of horizontal and vertical orientation preferences in developing ferret area 17. Proceedings of the National Academy of Sciences, 95, 2609-2614.

Chen, S., \& Levi, D. M. (1996). Meridional anisotropy in the discrimination of parallel and perpendicular lines - effect of body tilt. Perception, 25, 633-649.

Comerford, J. P., Javid, A. J., \& Thorn, F. (2000). Stimulus and head tilt reduce the Hermann Grid illusion. Investigative Ophthalmology \& Visual Science, S715.

Coppola, D. M., Purves, H. R., McCoy, A M., \& Purves, D. (1998). The distribution of oriented contours in the real world. Proceedings of the National Academy of Science USA, 95, 4002-4006.

Corballis, M. C., Anuza, T., \& Blake, L. (1978). Tachistoscopic perception under head tilt. Perception \& Psychophysics, 24(3), 274-284.

Corbett, J. E., \& Carrasco, M. (2011), Visual performance fields: Frames of reference. PLOS ONE, 6(9), 1-10.

Corbett, J. E., Handy, T. C., \& Enns, J. T (2009). When do we know which way is up? The time course of orientation perception. Vision Research, 49, 28-37.

Corwin, T. R., Moskowitz-Cook, A., \& Green, M. A. (1977). The oblique effect in a vernier acuity situation. Perception \& Psychophysics, 21(5), 445-449.

Daddaoua, N., Dicke, P. W., \& Their, P. (2014). Eye position information is used to compensate the consequences of ocular torsion on V1 receptive fields. Nature Communications, 5, 1-9. 
David, S. V., Vinje, W. E., \& Gallant, J. L. (2004). Natural image statistics alter the receptive field structure of V1 neurons. The Journal of Neuroscience, 24(31), 69917006.

deGardelle, V., Kouider, S., \& Sackur, J. (2010). An oblique illusion modulated by visibility: Non-monotonic sensory integration in orientation processing. Journal of Vision, 10(10), 1-9.

deWaele, C., Baudonniere, P., Lepecq, J., Tran Ba Huy, P., \& Vidal, P. (2001). Vestibular projections in the human cortex. Experimental Brain Research, 141, 541-551.

Denney, D., \& Adorjani, C. (1972). Orientation specificity of neurons after head tilt. Experimental Brain Research, 14, 312-317.

DeValois, Russell L., and Karen K. DeValois. "Multiple Spatial Frequency Channels." Spatial Vision. New York, NY [u.a.: Oxford Univ. Pr., 1988. 176-211. Print. Oxford Psychology Ser. No. 14.

Dieterich, M., \& Brandt, T. (2015). The bilateral central vestibular system: Its pathways, functions, and disorders. Annals of the New York Academy of Sciences, 1343, 1026.

Dyde, R. T., Jenkin, M. R., \& Harris, L. R. (2006). The subjective visual vertical and the perceptual upright. Experimental Brain Research, 173, 612-622.

Ebenholtz, S. M., \& Benzschawel, T. L. (1977). The rod and frame effect and induced head tilt as a function of observation distance. Perception \& Psychophysics, 22(5), 491-496.

Essock, EA (1980). The oblique effect of stimulus identification considered with respect to two classes of oblique effects. Perception, 9, 37-46. 
Essock, E. A., DeFord, J. K., Hansen, B. C., \& Sinai, M. J. (2003). Oblique stimuli are seen best (not worst!) in naturalistic broad-band stimuli: a horizontal effect. Vision Research, 43, 1329-1335.

Essock, E. A., Haun, A. M., \& Kim, Y. (2009). An anisotropy of orientation-tuned suppression that matches the anisotropy of typical natural scenes. Journal of Vision, 9(1). 1-15.

Essock, E. A., \& Siqueland, E. R. (1981). Discrimination of orientation by human infants. Perception, 10, 245-253.

Ferrante, D., Gerbino, W., \& Rock, I. (1995). Retinal vs. environmental orientation in the perception of the right angle. Acta Psychologica, 88, 25-32.

Freeman, J., Brouwer, G. J., Heeger, D. J., \& Merriam, E. P. (2011). Orientation coding depends on maps, not columns. The Journal of Neuroscience, 31(13), 4792-4804.

Frost, B. J., \& Kaminer, J. J. (1975). The orientation anisotropy and orientation constancy: A visual evoked potential study. Perception, 4, 51-58.

Furmanski, C. S., \& Engel, S. A. (2002). An oblique effect in primary visual cortex. Nature Neuroscience, 3(6), 535-536.

Girschik, A.R., Landy, M. S., \& Simoncelli, E. P. (2011). Cardinal Rules: Visual orientation perception reflects knowledge of environmental statistics. Nature Neuroscience 14, 926-932.

Graham, N., \& Sutter, A. (2000). Normalization: Contrast gain-control in simple (Fourier) and complex (non-Fourier) pathways of pattern vision. Vision Research, $40,2737-2761$. 
Guldin, W., \& Grüsser, O-J. (1998). Is there a vestibular cortex?. Trends in Neurosciences, 21, 254-259.

Hancock P. J. B., Baddeley R. J., \& Smith L. S. (1992). The principle components of natural images. Network: Computation in Neural Systems, 3, 61-70.

Hansen, B. C., \& Essock, E. A. (2004). A horizontal bias in human visual processing of orientation and its correspondence to the structural components of natural scenes. Journal of Vision, 4(12), 1044-1060.

Hansen, B.C., \& Essock, E. A. (2006). Anisotropic local contrast normalization: The role of stimulus orientation and spatial frequency bandwidths in the oblique and horizontal effect perceptual anisotropies. Vision Research, 46, 4398-4415.

Haslinger, R., Pipa, G., Lima, B., Singer, W., Brown, E. N., \& Neuenschwander, S. (2012). Context matters: The illusive simplicity of macaque V1 receptive fields. PLoS ONE, 7(7), 1-17.

Haun, A. M., Hansen, B. C., Kim, Y., \& Essock, E. A. (2005). Sequential effects and stimulus-response dependencies in an orientation identification task: Characterization of the class 2 oblique effect. Journal of Vision, 5(8): 158. Poster presented as Vision Sciences Society Annual Meeting, 2005.

Haun, A. M., \& Essock, E. A. (2010). Contrast sensitivity for orientated patterns in 1/f noise: Contrast response and the horizontal effect. Journal of Vision, 10(10), 1-20.

Heeley, D. W., \& Buchanan-Smith, H. M. (1992). Directional acuity for drifting plaids. Vision Research, 32, 97-104.

Hirsch, H. V., \& Spinelli, D. N. (1970). Visual experience modifies distribution of horizontally and vertically oriented receptive fields in cats. Science, 168, 869-871. 
Horn, G., Stechler, G., \& Hill, R. M. (1972). Receptive fields of units in the visual cortex of the cat in the presence and absence of bodily tilt. Experimental Brain Research, $15,113-132$.

Hubel, D. H., \& Wiesel, T. N. (1968). Receptive fields and functional architecture of monkey striate cortex. Journal of Physiology, 195, 215-243.

Jenkin, H. L., Dyde, R. T., Jenkin, M. R., Howard, I. P., \& Harris, L. R. (2003). Relative role of visual and non-visual cues in determining the direction of "up": Experiments in the York tilted room facility. Journal of Vestibular Research, 13, 287-293.

Jenkin, H. L., Dyde, R. T., Zacher, J. E., Zikovitz, D. C., Jenkin, M. R., Allison, R. S., Howard, I. P., \& Harris, L. R. (2005). The relative role of visual and non-visual cues in determining the perceived direction of "up": Experiments in parabolic flight. Acta Astronautica, 56, 1025-1032.

Jenkin, M. R., Dyde, R. T., Jenkin, H. L, Zacher, J. E., \& Harris, L. R. (2011). Perceptual upright: The relative effectiveness of dynamic and static images under different gravity states. Seeing and Perceiving, 24, 53-64.

Jin, J., Wang, Y., Swadlow, H. A., \& Alonso, J. M. (2011). Population receptive fields of $\mathrm{ON}$ and OFF thalamic inputs to an orientation column in visual cortex. Nature Neuroscience, 14, 232-238.

Kamitani, Y., \& Tong, F. (2005). Decoding the visual and subjective contents of the human brain. Nature Neuroscience, 8(5), 679-685.

Kayser, C., Kording, K., \& Konig, P. (2004). Processing of complex stimuli and natural scenes in the visual cortex. Current Opinions in Neurobiology, 14, 469-473. 
Keil, M. S., \& Cristóbal, G. (2000). Separating the chaff from the wheat: possible origins of the oblique effect. Journal of the Optical Society of America, 17, 697-710.

Kheradmand, A., Lasker, A., \& Zee, D. (2015). Transcranial magnetic stimulation (TMS) of the supramarginal gyrus: A window to perception of upright. Cerebral Cortex, $25,765-771$.

Kim, Y. J. Haun, A.M., \& Essock, E. A. (2010). The horizontal effect in suppression: Anisotropy overlay and surround suppression at high and low speeds. Vision Research 50(9), 838-849.

Lathan, C., Wang, Z., \& Clément, G. (2000). Changes in the vertical size of a threedimensional object drawn in weightlessness by astronauts. Neuroscience Letters, 295, 37-40.

Lee, B. B., Martin, P. R., \& Grünert, U. (2010). Retinal connectivity and primate vision. Progress in retinal and eye research, 29, 622-639.

Lee, B. B. (2011). Visual pathways and psychophysical channels in the primate. Journal of Physiology, 589, 41-47.

Leone, G., Lipshits, M., Gurfinkel, V., \& Berthoz, A. (1995). Influence of graviceptives cues at different level of visual processing: The effect of prolonged weightlessness. Acta Astronautica, 36, 743-751.

Leventhal, A. D., \& Hirsch, H. V. B. (1975). Cortical effect of early selective exposure to diagonal lines. Science, 190, 902-904.

Li, B., Peterson, M. R, \& Freeman, R. D. (2003). Oblique effect: A neural basis in the visual cortex. Journal of Neurophysiology, 90, 204-217. 
Lopez, C., Mercier, M. R., Halje, P., \& Blanke, O. (2011). Spatiotemporal dynamics of visual vertical judgments: Early and late brain mechanisms as revealed by high density electrical neuroimaging. Neuroscience, 181, 134-149.

Luyat, M., \& Gentaz, E. (2002). Body tilt effect on the reproduction of orientations: Studies on the visual oblique effect and subjective orientations. Journal of Experimental Psychology: Human Perception and Performance, 28(4), 1002 1011.

Luyat, M., Mobarek, S., Leconte, C., \& Gentaz, E. (2005). The plasticity of gravitational reference frame and the subjective vertical. Neuroscience Letters, 385, 215-219.

MacEvoy, S. P., Hanks, T. D., \& Paradiso, M. A. (2008). Macaque V1 activity during natural vision: Effects of natural scenes and saccades. Journal of Neurophysiology, $99,460-472$.

Maffei, L. Campbell, F. W. (1970). Neurophysiological location of the vertical and horizontal visual coordinates in man. Science, 167, 386-387.

Maloney, R. T. \& Clifford, C. (2015). Orientation anisotropies in human primary visual cortex depend on contrast. NeuroImage, 119, 129-145.

Mannion, D. J., McDonald, J. S., \& Clifford, C. (2010). Orientation anisotropies in human visual cortex. Journal of Neurophysiology, 103, 3465-3471.

Mansfield, R. J. W. (1974). Neural basis of orientation in primate vision. Science, 186, 1133-1135.

Mansfield, R. J. W., \& Ronner, S. F. (1978). Orientation anisotropy in monkey visual cortex. Brain Research, 149, 229-234. 
Marendaz, C., Stivalet, P., Barraclough, L., \& Walkowiac, P. (1993). Effect of Gravitational cues on visual search for orientation. Journal of Experimental Psychology: Human Perception and Performance, 19(6), 1266-1277.

McIntyre, J., Lipshits, M., Zaoui, M., Berthoz A., \& Gurfinkel, V. (2001). Internal reference frames for representation and storage of visual information: The role of gravity. Acta Astronautica, 49, 111-121.

Meng, X., \& Qian, N. (2005). The oblique effect depends on perceived, rather than physical, orientation and direction. Vision Research, 45, 3402-3413.

Mertz, S., \& Lepecq, J. (2001). Imagined body orientation and perception of the visual vertical, Psychological Research, 65, 64-70.

Mikhailova, E. S., Gerasimenko, N. Y., Krylova, M. A., Izyurov, I. V., \& Slavutskaya, A. V. (2015). Mechanisms of orientation sensitivity of human visual system: Part II. Neural patterns of early processing of information about line orientation. Human Physiology, 41(3), 229-241.

Nasr, S., \& Tootell, R. B. H. (2012). A cardinal orientation bias in scene-selective visual cortex. The Journal of Neuroscience, 32(43), 14921-14926.

Petro, L., Vizioli, L., \& Muckli, L. (2014). Contributions of cortical feedback to sensory processing in primary visual cortex, Frontiers in Psychology, 5, 1-8.

Prather, J. R. (1997). Phenomenological cue effects on the class II oblique effect (Unpublished doctoral dissertation). University of Louisville, Kentucky.

Priebe, N. J., \& Ferster, D. (2012). Mechanisms of neuronal computation in mammalian visual cortex. Neuron, 75, 194-208. 
Rancz, E., Moya, J., Drawitsch, F., Brichta, A., Canals, S., \& Margrie, T. (2015).

Widespread vestibular activation of the rodent cortex. The Journal of Neuroscience, 35, 5926-5934.

Reinis. S., Landolt, J. P., Money, K. E., Lahue, R. H., \& Weiss, D. S. (1986). Effect of head tilt on visual cortical cell function in the intact and labyrinthectomized cat. Experimental Neurology, 91, 102-126.

Rose, D., \& Blakemore, C. (1974). An analysis of orientation selectivity in the cat's visual cortex. Experimental Brain Research, 20, 1-17.

Sanders, R. D., \& Gillig, P. M. (2010). Cranial nerve VIII: Hearing and vestibular functions. Psychiatry, 7(3), 17-22.

Sauvan, X. M., Peterhans, E. (1999). Orientation constancy in neurons of monkey visual cortex. Visual Cognition, 6(1), 43-54.

Schwartz O., \& Simoncelli E. P. (2001). Natural signal statistics and sensory gain control. Nature Neuroscience, 4, 819-825.

Schweinhart, A., Shafto, P. and Essock, E. Effects of recent exposure to atypical environmental statistics on orientation perception: analyzing the plasticity of the horizontal effect. F1000Posters 2015, 6:474 (poster).

Shapley, R. (2009). Linear and nonlinear systems analysis of the visual system: Why does it seem so linear? A review dedicated to the memory of Henk Spekreijse. Vision Research, 49, 907-921.

Shen, G., Tao, X., Zhang, B., Smith, E. L., \& Chino, Y. M. (2014). Oblique effect in visual area 2 of macaque monkeys. Journal of Vision, 14(2), 1-16. 
Snyder, L. (1999). This way up: Illusions and internal models in the vestibular system. Nature Neuroscience, 2(5), 396-398.

Smyrnis, N., Mantas, A., \& Evdokimidis, I. (2014). Two independent sources of anisotropy in the visual representation of direction in 2-D space. Experimental Brain Research, 232, 2317-2324.

Sun, M., Huang, J., Wang, F., An, A., Tian, F., Liu, H., Niu, H., \& Song, Y. (2013). Quantitative comparison of the hemodynamic activation elicited by cardinal and oblique gratings with functional near-infrared spectroscopy. NeuroReport, 24, 354358.

Sun, P., Gardner, J. L, Costagli, M., Ueno, K., Waggoner, R. A., Tanaka, K., \& Cheng, K. (2013). Demonstration of tuning to stimulus orientation in the human visual cortex: A high-resolution fMRI study with a novel continuous and periodic stimulation paradigm. Cerebral Cortex, 23(7), 1618-1629.

Switkes, E., Mayer, M. J., \& Sloan J. A. (1978). Spatial frequency analysis of the visual environment: Anisotropy and the carpentered environment hypothesis. Vision Research, 18, 1393-1399.

Tanaka, S., Tani, T., Ribot, J., O’Hashi, K., \& Imamura, K. (2009). A postnatal critical period for orientation plasticity in the cat visual cortex. PLoS ONE, 4(4), 1-10.

Tarnutzer, A. A., Bockisch, C. J., \& Straumann, D. (2009). Head roll dependent variability of subjective visual vertical and ocular counterroll. Experimental Brain Research, 195, 621-626. 
Tarnutzer, A. A., Fernando, D. P., Kheradmand, A., Lasker, A. G., \& Zee, D. S. (2012). Temporal constancy of perceived direction of gravity assessed by visual line adjustments. Journal of Vestibular Research, 22, 41-54.

Teller, D. Y., Morse, R., Borton, R., \& Regal, D. (1974). Visual acuity for vertical and diagonal gratings in human infants. Vision Research, 14, 1433-1439.

Tyler, C. W. \& Mitchell, D. E. (1977). Orientation differences for perception of sinusoidal line stimuli. Vision Research, 17, 83-88.

Wade, J. (1992). The representation of orientation in vision. Australian Journal of Psychology, 44(3), 139-145.

Wandell, B. (1995). Foundations of vision. Sunderland, Mass.: Sinauer Associates.

Wang, G., Ding, S. \& Yunokuchi, K. (2003). Difference in the representation of cardinal and oblique contours in cat visual cortex. Neuroscience Letters, 338, 77-81.

Wainwright, M. J. (1999) Visual adaptation as optimal information transmission. Vision Research, 39(23), 3960-3974.

Westheimer, G. (2003). Meridional anisotropy in visual processing: Implications for the neural site of the oblique effect. Vision Research, 43, 2281-2289.

Williams, R. A., Boothe, R. G., Kiorpes, L., \& Teller, D. Y. (1981). Oblique effects in normally reared monkeys (macaca nemestrina): Meridional variations in contrast sensitivity measured with operant techniques. Vision Research, 22, 1253-1266.

Willmore, B. D. B., Bulstrode, H., \& Tolhurst, D. J. (2012). Contrast normalization contributes to a biologically-plausible model of receptive-field development in primary visual cortex (V1). Vision Research, 54, 49-60. 
Yacoub, E., Harel, N., \& Ugurbil, K. (2008). High-field fMRI unveils orientation columns in humans. PANAS, 105(30), 10607-10612. 


\section{LIST OF COPYRIGHT APPROVAL}

FIGURE 1

License \#

License Content Publisher

License Content Publication

License Content Title

License Content Author

License Content Date

License Content Pages
4097260634330

John Wiley and Sons

Journal of Physiology

Visual pathways and psychophysical channels in the primate

Barry B. Lee

Dec 23, 2010

7

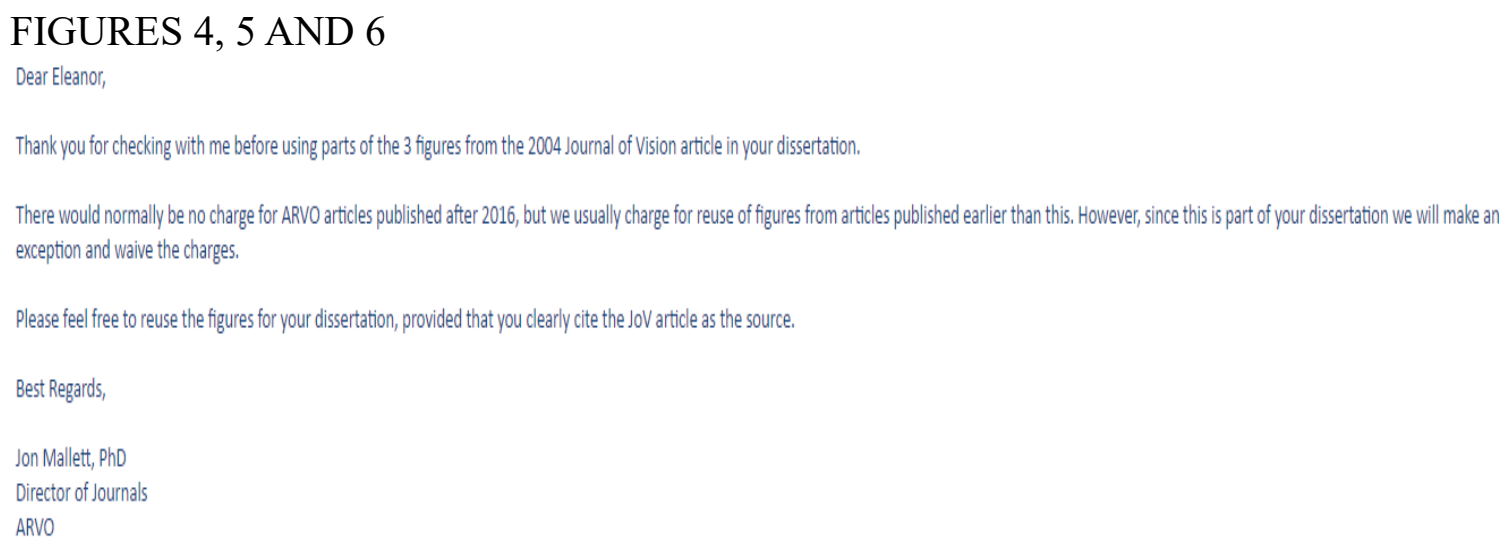

Hansen, B. C., \& Essock, E. A. (2004). A horizontal bias in human visual processing of orientation and its correspondence to the structural components of natural scenes. Journal of Vision, 4(12), 1044-1060.

FIGURE 8

License \#

License Content Publisher

License Content Publication

License Content Title

License Content Author

License Content Date

License Content Pages
4097260940009

Elsevier

Vision Research

When do we know which way is up? The time course of orientation perception Jennifer E. Corbett, Todd C. Handy, James T. Enns January 2009

49 


\section{FIGURE 19}

F1000Research articles are usually published under a CC-BY license, which permits unrestricted use, distribution, and reproduction in any medium, provided the original work is properly cited, and leaves the copyright of the article with the current copyright holder (usually the author or their institution). Additional waivers are used for some governmental employees, as appropriate. As the specific version of the CC-BY license applied may change due to periodic updates, the copyright information is shown below the abstract.

Most posters, slides and documents are also published under a CC BY license, but other CC licenses may apply, as indicated on each research output's published page under the image.

Schweinhart, A., Shafto, P. and Essock, E. Effects of recent exposure to atypical environmental statistics on orientation perception: analyzing the plasticity of the horizontal effect. F1000Posters 2015, 6:474 (poster). 


\section{CURRICULUM VITAE}

Eleanor O'Keefe

PHONE: (510) 439-8455

ADDRESS: 551 LILLY AVE, LOUISVILLE, KY. 40217

E-MAIL: EMAYOKEEFE@GMAIL.COM

\section{EDUCATION}

\section{PhD in Psychological and Brain Sciences}

University of Louisville, Louisville, KY, will be completed early 2017

Dissertation: Psychophysical analysis of three separate anisotropic patterns in vision:

Putting visual reference frames in conflict to study the Class 1 and 2 oblique effects and the horizontal effect

MS in Psychology and Brain Sciences

University of Louisville, Louisville, KY, June 2013

Bachelor of Science in Bio-Psychology with Minor in Applied Psychology

University of California Santa Barbara, Santa Barbara, CA, 2010

Dean's List, 2007-2010

\section{RESEARCH EXPERIENCE}

PhD student in Visual Perception Lab, July 2011-Present

University of Louisville

Louisville, KY

- Use psychophysical methods to investigate both how the visual system processes natural scene statistics and what reference frames the system uses to define stimulus orientation

- Mentor undergraduate students as research assistants in the lab. Ran monthly lab meetings where students were given an opportunity to discuss journal articles, give presentations and talk in-depth about current lab research.

- Write both original code and edited code in MatLab and the psychophysics toolbox for all experiments

- Constructed an apparatus that enables head tilt of 45 degrees, while supporting head and chin, as well as integrates dove prisms to enable visual scene tilt

- Investigated the role of temporally-modulated stimuli with regards to stimulus orientation and both overlay and surround suppression

- Helped configure camera attachment to head mounted device for adaptation experiments

- Wrote and received consent for my studies through the University's IRB. This including writing experimental protocol, informed consent documents and flyers for participant recruitment as well as keeping up with continuing review and amendments as needed. 


\section{Research Assistant in Affective Cognitive Neuroscience Lab, August 2010-June}

2011

University of California Berkeley

Berkeley, CA

- Completed university fMRI safety training, recruited subjects for fMRI sessions and assisted postdocs during fMRI sessions

- Traced amygdala on fMRI scans in FSLview for voxel analysis

- Coded image database

Research Assistant in LABB (attention and behavior research lab), JanuaryDecember 2009

University of California Santa Barbara

- Completed lab EEG training, assisted postdocs during EEG sessions including use of Zebris electrode localization system

- Recruited and ran participants in psychophysical gaze-cueing experiments

Research Assistant in RECVEB (social behavior virtual reality lab), June 2008June 2009

University of California Santa Barbara

- Recruited and ran participants in social psychology experiments involving questionnaires, EKG, and virtual reality equipment (HMD, InterSense)

\section{PRESENTATIONS}

O’Keefe, E., Essock, E., \& DeMarco, P. (2015). Putting visual reference frames in conflict to study the horizontal effect: a visual anisotropy. F1000Research, 4: 725; doi: 10.7490/f1000research.1110473.1.

O’Keefe, E.M., Kim, Y., \& Essock, E.A. (2013). Differential properties of NarrowlyTuned and Broad Temporal Channels. Journal of Vision, 13(9): 272; doi: $10.1167 / 13.9 .272$

Schweinhart, A.M., Dubinchik, M., O’Keefe, E.M., Williams, H.G., \& Essock, E.A. (2012). Regularities in the Anisotropic Content of Portrait and Landscape Paintings: A Corollary to the Horizontal Effect Anisotropy of Visual Processing. Journal of Vision 12(9); doi: 10.1167/12.9.842.

Williams, H.G., Schweinhart, A.M., O’Keefe, E.M., Haun, A.M., \& Essock, E.A. (2012). Aesthetic Preference of Oriented Content: Relation to the Anisotropic Content of Natural Scenes. Journal of Vision, 12(9); 1084; doi:

10.1167/12.9.842. 


\section{GRANTS AND FELLOWSHIPS}

University of Louisville Arts and Sciences Creative Activities Grant, 2016

\section{Graduate Research Funds}

University of Louisville College of Arts and Sciences Graduate Student Council, 2013, 2016

University of Louisville College of Arts and Sciences Graduate Student Union, 2011

\section{University Fellowship, 2011-2013}

University of Louisville College of Arts and Sciences

\section{TEACHING EXPERIENCE}

Graduate Teaching Assistant, August 2013-June 2016

University of Louisville

Louisville, KY

Introduction to Psychology

Personality

Sensation and Perception

Experimental Psychology (taught lab sections)

Lifespan Developmental Psychology

\section{Guest Lecturer}

University of Louisville

Louisville, KY

Sensation and Perception, March 2014

Introduction to Psychology, June and September 2016

\section{SUMMARY OF QUALIFICATIONS}

Computer Experience - experienced in Windows 7/10/XP, Microsoft Office particularly Excel, MatLab (including psychophysics toolbox), SONA systems, SPSS and $\mathrm{C} / \mathrm{C}^{++}$.

Lab Experience - experienced in general lab work, experimental procedure, keeping experimental records, biotechnology equipment, virtual reality equipment, construction, monitor calibration and photometric measurement.

Research Experience - experienced in psychophysical methods, experimental design and IRB protocol, EEG, EKG, fMRI, participant recruitment and instruction, and statistical analysis.

Departmental Involvement - student representative of the Department of Psychological and Brain Sciences, University of Louisville (2012-2014). 